\title{
Ordered analysis of heavy flavor production in deep-inelastic scattering
}

\author{
R. S. Thorne and R. G. Roberts \\ Rutherford Appleton Laboratory, Chilton, Didcot, Oxon., OX11 OQX, United Kingdom
}

(Received 29 September 1997; published 16 April 1998)

\begin{abstract}
At low $Q^{2}$, charm production in deep-inelastic scattering is adequately described by assuming generation in electroweak boson-light parton scattering (dominantly boson-gluon fusion), which naturally incorporates the correct threshold behavior. At high $Q^{2}$ this description is inadequate, since it does not sum $\operatorname{logs}$ in $Q^{2} / \mathrm{m}_{c}^{2}$, and is replaced by the treatment of the charm quark as a light parton. We show how the problem of going from one description to the other can be solved in a satisfactory manner to all orders. The key ingredient is the constraint of matching the evolution of the physical structure function $F_{2}$ order by order in $\alpha_{s}\left(Q^{2}\right)$, in addition, to the matching of the value of $F_{2}$ itself. This leads to new expressions for the coefficient functions associated with the charm parton, which are unique in incorporating both the correct threshold and asymptotic behaviors at each order in perturbation theory. The use of these improved coefficients leads to an improvement in global fits and an excellent description of the observed $F_{2, \text { charm }}$. [S0556-2821(98)05709-9]

PACS number(s): 13.60.Hb, 11.10.Gh, 12.38.Bx
\end{abstract}

\section{INTRODUCTION}

The factorization theory in QCD [1] has existed for many years, and has been one of the triumphs of quantum field theory. However, in its original form it does not take account of a number of possible complications; i.e., it exists only for massless particles, and its ordering does not take account of possible enhancements at high orders in $\alpha_{s}$ at small $x$. Until the past few years, both of these complications were not of any real phenomenological importance. The lowest values of $x$ probed were large enough that it was unimportant to consider small $x$ enhancement. Also, the up, down, and strange quarks were considered light enough to be treated as massless, whenever one was within the realm of perturbative QCD. Furthermore, there were little data on the charm contribution to the structure function and it was generally such a small component of the total structure function that it could be treated very approximately.

Both of the above complications have recently become a great deal more important due to the advent of the DESY ep collider HERA. This now probes structure functions at far lower values of $x$ than any previous experiments, as low as $x \sim 10^{-5}[2,3]$, and the treatment of structure functions should really take proper account of any small $x$ complications. Also, the small $x$ structure functions now have a contribution due to the charm structure function, which is far from insignificant, i.e., it can be more than $20 \%$ of the total structure function and, moreover, in the past couple of years direct measurement of the charm structure function has also become possible $[4,5]$. This has made it essential to treat the contribution to the structure function due to massive quarks in a correct manner.

In this paper we propose a new method for the treatment of heavy quarks in structure functions. We begin by describing the features a correct treatment must exhibit at both high and low $Q^{2}$, and the techniques used in either of these limits. We then give a discussion of the correct way to take account of heavy quarks in a well-ordered manner over the full range of $Q^{2}$, showing how this relates to present treatments, and in particular demonstrating that one may choose to evolve the partons according to the massless evolution equations. However, we shall see that the detailed construction of the coefficient functions required is extremely difficult if not impossible. Therefore, we provide instead a prescription for calculating structure functions including heavy quark effects, which is somewhat simpler than the strictly correct treatment, and which is directly analogous to the normal manner in which one calculates order by order for massless partons, but which is in practice essentially identical to the strictly correct treatment. Finally, we will present the results of a comparison of our method to data: both that for full structure functions and for the charm component to the structure functions. These comparisons turn out to be very good. We also make predictions for the charm component of the longitudinal structure function. Complications due to leading $\ln (1 / x)$ terms at all orders in $\alpha_{s}$ are ignored, and while a correct treatment of structure functions should of course deal with this problem, we feel that this would overcomplicate our presentation, and besides we wish to compare directly with normal next-to-leading order (NLO) in $\alpha_{s}$ approaches. A paper which takes account of both small $x$ complications and massive partons is in preparation.

\section{STRUCTURE FUNCTIONS WITH MASSIVE QUARKS}

We consider the case of $n_{f}$ massless quarks and one heavy quark. One of the simplest ways to deal with heavy flavor production in deep-inelastic scattering is to treat the mass of the heavy quark, $M$, as a hard scale [6]. In this case the $n_{f}$ light quarks are always treated as partons, but all other quarks are never treated as partons at any scale: the cross section for production of heavy quarks is expressed entirely in terms of coefficient functions depending on the heavy quark mass convoluted with parton distributions which only depend on light partons, i.e.,

$$
\begin{aligned}
\sigma_{i}\left(x, Q^{2}, M^{2}\right)= & \sum_{a} C_{i a}^{\mathrm{FF}}\left(Q^{2} / \mu^{2}, Q^{2} / M^{2}\right) \otimes f_{a}^{n_{f}}\left(\mu^{2}\right) \\
& +\mathcal{O}\left(\Lambda^{2} / M^{2}\right)
\end{aligned}
$$


where $\sigma_{i}\left(x, Q^{2}, M^{2}\right)$ is the cross section for scattering off a particular quark, either heavy or light, and $a$ runs over the light partons, i.e., the gluon and the $n_{f}$ light quarks. This approach is very well-defined in theoretical terms, essentially being a simple generalization of the usual factorization theorem, with Eq. (2.1) being valid to all orders up to the higher twist corrections of $\mathcal{O}\left(\Lambda^{2} / M^{2}\right)$.

This approach is adopted by a number of groups [7], and is usually known as the fixed flavor number scheme (FFNS). It is normally used in the particular renormalization scheme, where all diagrams with no heavy quark lines are renormalized in the modified minimal subtraction $(\overline{\mathrm{MS}})$ scheme, while those with heavy quarks are renormalized at zero momentum. This is particularly convenient because the effect of the heavy particle decouples from the light sector, in particular the coupling is the three flavor $\overline{\mathrm{MS}}$ coupling and the light parton distributions evolve as in the three flavor $\overline{\mathrm{MS}}$ scheme. The $C_{i a}^{\mathrm{FF}}\left(z, Q^{2} / \mu^{2}, Q^{2} / M^{2}\right)$ have all been calculated to $\mathcal{O}\left(\alpha_{s}\right)[6,8,9]$ and $\mathcal{O}\left(\alpha_{s}^{2}\right)[10]$ in this scheme, though analytic expressions only exist at $\mathcal{O}\left(\alpha_{s}\right)$.

In principle this approach is a very good way to calculate the effects of heavy quarks in structure functions. At each order it incorporates the kinematical threshold in the light parton-photon center of mass energy $\hat{W}^{2} \equiv Q^{2}\left(z^{-1}-1\right)$ $\geqslant 4 M^{2}$ in a smooth manner (which then guarantees the same smooth threshold in the invariant mass of the hadronic remnant $W^{2}$, up to proton mass corrections) and the coefficient functions are calculated order by order in precisely the same manner as the light particle coefficient functions (though the actual calculations are rather more difficult). However, it does have one major shortcoming. As one calculates to higher orders in $\alpha_{s}$, one encounters higher powers of $\ln \left(Q^{2} / M^{2}\right)$ and $\ln \left(\mu^{2} / M^{2}\right)$. Letting $\mu^{2}=Q^{2}$, and thus eliminating all logs in $Q^{2} / \mu^{2}$, then for $Q^{2} \rightarrow \infty$ the coefficients at $m$ th-order in $\left[\alpha_{s}\left(Q^{2}\right) / 2 \pi\right]^{m}$ have the series expansion

$$
C_{i a}^{\mathrm{FF}, m}\left(z, Q^{2} / M^{2}\right)=\sum_{n=0}^{m} f_{n}(z) \ln ^{n}\left(Q^{2} / M^{2}\right)
$$

Thus, working order by order in $\alpha_{s}$ in this approach, one is failing to take account of these large logs. This is not only a practical concern in the sense that these large logs in $Q^{2} / M^{2}$ at higher orders in $\alpha_{s}$ can potentially be phenomenologically important, ${ }^{1}$ but is also a theoretical concern insofar as at each order in $\alpha_{s}$ the leading power of $\ln \left(Q^{2} / M^{2}\right)$ is the leading in $\alpha_{s}$ part of the overall coefficient function with this $\ln \left(Q^{2} / M^{2}\right)$ behavior, and is really part of the leading-order expression for the structure function as a whole. The same reasoning applies for the next-to-leading power of $\ln \left(Q^{2} / M^{2}\right)$, etc. This is similar in principle to the problem of increasing powers of $\ln (1 / x)$ with increasing powers of $\alpha_{s}$. It is more difficult in one sense, in so much that in the expressions for the coefficient functions, these large logs in $\left(Q^{2} / M^{2}\right)$ are hidden within very complicated expressions. However, it is far simpler in the particular limit $Q^{2} \gg M^{2}$ because we know exactly

\footnotetext{
${ }^{1}$ They are not important for $Q^{2} \ll M^{2}$ because the large logs are destroyed by factors coming from the kinematical threshold.
}

how to sum the logarithms in $Q^{2}$, i.e., we solve the renormalization group equation for fixed order in $\alpha_{s}$.

Thus, in order to sum these large logs in $Q^{2} / M^{2}$, it is convenient to consider the heavy quark to be a parton and for its distribution function to satisfy the renormalization group [Dokshitzer-Gribov-Lipatov-Altarelli-Parisi (DGLAP)] equations as do the light partons. An extremely simple approach which incorporates this idea is the zeromass variable flavor number scheme (ZM-VFNS). This treats the massive parton as being infinitely massive below some threshold in $\mu^{2}$, and totally massless above the threshold, i.e., all coefficient functions coupling directly to the charm quark turn on at the threshold, the evolution of the charm quark begins at this threshold, and the number of flavors in the coefficient functions, anomalous dimensions and the running coupling constant increases by one to $n_{f}+1$ discontinuously at the threshold. Despite the simplicity of the approach, this procedure must in principle be done with care if the correct results are to be obtained in the asymptotic limits [11] (see below for details). In particular, the decoupling theorem tells one how the coupling constant must change in order to get the correct results well below threshold. Also, the parton distributions just above the chosen threshold must be carefully defined in terms of those below threshold in order to guarantee that the correct result is obtained as $Q^{2} \rightarrow \infty$. In practice at low orders the situation is relatively simple, e.g., if the threshold is chosen to be precisely $\mu^{2}=M^{2}$, then at NLO, the light parton distributions are continuous across the threshold (in $\overline{\mathrm{MS}}$ scheme) and the evolution of the charm parton distribution begins from zero. At higher orders the parton distributions must change discontinuously across the threshold and in particular the charm evolution must begin from a nonzero value.

For many years the above approach was that most commonly used in global fits. The collaboration on theoretical and experimental QCD (CTEQ) used the approach at NLO, as explained above [12], while the Martin-Roberts-Stirling (MRS) collaboration motivated their choice of threshold by phenomenological considerations rather than the strict theoretical ones [13], but in practice this resulted in a very similar choice of threshold (i.e., $2.7 \mathrm{GeV}^{2}$ for MRS compared to $2.56 \mathrm{GeV}^{2}$ for CTEQ). While the charm contribution to the structure functions near the region of threshold was not too important, this simple treatment was perfectly adequate. However, it is clear from its construction that it will not suffice as a good description of charm production in the region of the charm threshold. In particular charm production has a sharp threshold at a chosen $\mu^{2}$, rather than a smooth threshold in $W^{2}$.

Hence, some approach which extrapolates smoothly from the FFNS at low $Q^{2}$ to the ZM-VFNS at high $Q^{2}$ is required in order to produce a good description of the effect of heavy quarks on structure functions over the whole range of $Q^{2}$. Let us discuss how this may be achieved. In order to do this, we first put the ZM-VFNS on a more solid theoretical footing. If we regard the quark mass $M^{2}$ as being a soft scale, then the factorization theorem tells us that

$$
\begin{aligned}
\sigma_{i}\left(x, Q^{2}, M^{2}\right)= & \sum_{b} C_{i b}^{n_{f}+1}\left(Q^{2} / \mu^{2}\right) \otimes f_{b}^{n_{f}+1}\left(\mu^{2}, M^{2} / \mu^{2}\right) \\
& +\mathcal{O}\left(M^{2} / Q^{2}\right)
\end{aligned}
$$


where $b$ runs over the light partons and the massive quark. We are able to remove the large logs in $Q^{2} / M^{2}$ from the coefficient functions, and hence obtain the normal massless coefficient functions, and absorb them into the definition of the parton distributions at the expense of having potential "higher twist" corrections of $\mathcal{O}\left(M^{2} / Q^{2}\right)$. While the parton distributions depend on $M^{2}$, if the operators defining the partons undergo ultraviolet operator regularization in the $\overline{\mathrm{MS}}$ scheme, then their evolution depends only on the anomalous dimensions obtained from this ultraviolet regularization. These are independent of the mass of the heavy parton, and the evolution is as if for $n_{f}+1$ massless quarks in the $\overline{\mathrm{MS}}$ scheme. Hence we have the formal definition of the ZMVFNS, which will become exact for $Q^{2} \gg M^{2}$.

However, we have one more degree of freedom in Eq. (2.3) than in Eq. (2.1), i.e., we have the heavy parton distribution to parametrize at some arbitrary starting scale for evolution, and also no apparent reference to the mass scale $M^{2}$ in the definition of the parton distributions. This is not, in fact, true, since it can be shown that

$$
f_{b}^{n_{f}+1}\left(z, \mu^{2}, M^{2} / \mu^{2}\right)=A^{b a}\left(M^{2} / \mu^{2}\right) \otimes f_{a}^{n_{f}}\left(\mu^{2}\right),
$$

where the operator matrix elements $A^{b a}\left(z, \mu^{2} / M^{2}\right)$ contain logs in $\left(\mu^{2} / M^{2}\right)$, and are calculable order by order in perturbation theory $[14,15]$. [We denote the matrix elements relating the heavy quark distribution to the $n_{f}$-flavor light parton distributions as $A^{H a}\left(z, \mu^{2} / M^{2}\right)$ and those relating the $\left(n_{f}\right)$-flavor light parton distributions to the $n_{f}$-flavor light parton distributions as $A^{b a, H}\left(z, \mu^{2} / M^{2}\right)$ as in the above references.] Hence, the partons in the ZM-VFNS can in fact be generated from those in the FFNS at all $\mu^{2}$ by using the leading logarithmic expressions for the operator matrix elements and the expression (2.4), rather than using the fourflavor evolution equations at all. Indeed, if the starting scale is chosen as $\tilde{\mu}^{2} \neq M^{2}$, then strictly speaking all the leading logs in $\left(\tilde{\mu}^{2} / M^{2}\right)$ should be included in the matching condition, which is just as complicated as using Eq. (2.4) at all scales. However, if the scale at which evolution begins is precisely $\mu^{2}=M^{2}$, then the matching condition for the partons in the two schemes is a power series in $\alpha_{s}$ with no logs. Therefore, it simplest to use Eq. (2.4) only to define the order-by-order parton distributions at the starting scale, and then to calculate the parton distributions at other scales by evolving using $n_{f}+1$ massless flavors. This procedure guarantees the correctness of the ZM-VFNS calculation in the limit $Q^{2} \gg M^{2}$.

By comparing the expressions (2.3) and (2.1) at $Q^{2} \gg M^{2}$, and using the relationship (2.4), one can calculate the FFNS coefficient functions, up to $\mathcal{O}\left(M^{2} / Q^{2}\right)$ corrections in terms of the massless $\overline{\mathrm{MS}}$ coefficient functions for $n_{f}+1$ flavors $[14,15]$, i.e.,

$$
\begin{aligned}
C_{i a}^{\mathrm{FF}}\left(z, Q^{2} / \mu^{2}, Q^{2} / M^{2}\right)= & C_{i b}^{n_{f}+1}\left(Q^{2} / \mu^{2}\right) \otimes A^{b a}\left(\mu^{2} / M^{2}\right) \\
& +\mathcal{O}\left(M^{2} / Q^{2}\right) .
\end{aligned}
$$

The detailed expressions of this form can be found in [15], where they are used to calculate the $Q^{2} \rightarrow \infty$ limit of the heavy quark coefficient functions in terms of the known light quark coefficient functions and calculated operator matrix elements. These authors then define $F^{\mathrm{ASYMP}}$ as the structure function obtained from these asymptotic expressions for the coefficient functions and the parton distribution in Eq. (2.1). They then, through purely phenomenological motivation, define a variable flavor number scheme $[15,16]$ by the formal definition

$$
F^{\mathrm{VFNS}}=F^{\mathrm{ZM}-\mathrm{VFNS}}-F^{\mathrm{ASYMP}}+F^{\mathrm{FFNS}} .
$$

This then extrapolates smoothly from one limit to the other, being guaranteed to reduce to the correct limit order by order in $\alpha_{s}\left(Q^{2}\right)$ at high $Q^{2}$, though only approximately to $F^{\mathrm{FFNS}}$ order by order at low $Q^{2}$.

\section{A COMPLETE TREATMENT OF CHARM MASS CORRECTIONS}

Although we agree with Eq. (2.5) and hence with the results at high $Q^{2}$ regarding coefficient functions in [14,15], we believe one may be more ambitious. Rather than simply accepting the uncertainty of $\mathcal{O}\left(M^{2} / Q^{2}\right)$ in Eq. (2.5), we can be more systematic and demand that there is a scheme, which uses the definition of the parton distributions in Eqs. (2.3) and (2.4), but which is correct up to $\mathcal{O}\left(\Lambda^{2} / M^{2}\right)$. Inserting Eq. (2.4) into Eq. (2.3) and subtracting from Eq. (2.1), it is clear that the difference between the FFNS and the ZMVFNS, i.e., the error in the latter, is given by

$$
\begin{aligned}
c_{i}^{a}\left(M^{2} / \mu^{2}\right) \otimes f_{a}^{n_{f}}\left(\mu^{2}\right)= & {\left[C_{i a}^{\mathrm{FF}}\left(Q^{2} / \mu^{2}, Q^{2} / M^{2}\right)\right.} \\
& \left.-C_{i b}^{n_{f}+1}\left(Q^{2} / \mu^{2}\right) \otimes A^{b a}\left(\mu^{2} / M^{2}\right)\right] \\
& \otimes f_{a}^{n_{f}}\left(\mu^{2}\right)
\end{aligned}
$$

where $c_{i}^{a}\left(z, M^{2} / Q^{2}\right)$ is representative of the error in the ZMVFNS and is of $\mathcal{O}\left(M^{2} / Q^{2}\right)$. However, making use of Eq. (2.4) this difference can be written as

$$
c_{i}^{a}\left(M^{2} / \mu^{2}\right) \otimes\left(A^{b a}\left(M^{2} / \mu^{2}\right)\right)^{-1} \otimes f_{b}^{n_{f}+1}\left(\mu^{2}, \mu^{2} / M^{2}\right),
$$

and so $c_{i}^{a}\left(M^{2} / \mu^{2}\right) \otimes\left(A^{b a}\left(M^{2} / \mu^{2}\right)\right)^{-1}$ is precisely the correction to the massless ZM-VFNS coefficient functions, which is required to correct for the $\mathcal{O}\left(M^{2} / Q^{2}\right)$ errors in this scheme. Thus, defining corrected $n_{f}+1$-flavor coefficient functions by

$$
\begin{aligned}
C_{i b}^{\mathrm{VF}}\left(z, Q^{2} / \mu^{2}, Q^{2} / M^{2}\right)= & C_{i b}^{n_{f}+1}\left(z, Q^{2} / \mu^{2}\right)+c_{i}^{a}\left(M^{2} / \mu^{2}\right) \\
& \otimes\left(A^{b a}\left(M^{2} / \mu^{2}\right)\right)^{-1} \\
= & C_{i a}^{\mathrm{FF}}\left(Q^{2} / \mu^{2}, Q^{2} / M^{2}\right) \\
& \otimes\left(A^{b a}\left(\mu^{2} / M^{2}\right)\right)^{-1}
\end{aligned}
$$

or alternatively

$$
\begin{aligned}
& C_{i b}^{\mathrm{VF}}\left(z, Q^{2} / \mu^{2}, Q^{2} / M^{2}\right) \otimes A^{b a}\left(\mu^{2} / M^{2}\right) \\
& \quad=C_{i a}^{\mathrm{FF}}\left(Q^{2} / \mu^{2}, Q^{2} / M^{2}\right),
\end{aligned}
$$

and demanding that the VFNS coefficient functions satisfy this equality at all $Q^{2}$, then our VFNS is guaranteed to give 
exactly the same all-orders result as the FFNS. Hence, we have the factorization theorem

$$
\begin{aligned}
\sigma_{i}\left(x, Q^{2}, M^{2}\right)+\mathcal{O}\left(\Lambda^{2} / M^{2}\right)= & \sum_{b} C_{i b}^{\mathrm{VF}}\left(Q^{2} / \mu^{2}, M^{2} / \mu^{2}\right) \\
& \otimes f_{b}^{n_{f}+1}\left(\mu^{2}, M^{2} / \mu^{2}\right) \\
\equiv & \sum_{a} C_{i a}^{\mathrm{FF}}\left(Q^{2} / \mu^{2}, M^{2} / \mu^{2}\right) \\
& \otimes f_{a}^{n_{f}}\left(\mu^{2}\right) .
\end{aligned}
$$

Thus, Eq. (3.5) gives us a method for defining the structure function including a heavy quark, which when written in terms of the $n_{f}$ flavor parton densities $f_{a}^{n_{f}}\left(\mu^{2}\right)$ is identical at each order in $\alpha_{s}$ to the FFNS, and thus is correct up to errors of $\mathcal{O}\left(\Lambda^{2} / M^{2}\right)$, but where all partons evolve according to the massless evolution equations and thus all $\operatorname{logs}$ in $\mu^{2} / M^{2}$ are automatically summed correctly.

Bearing in mind this above result, it seems sensible that the best way to proceed for the calculation of structure functions in the presence of a heavy quark is to use the FFNS up to some scale of $\mathcal{O}\left(M^{2}\right)$ and then switch to the scheme defined by Eq. (3.5) above this scale. (Changes of renormalization scheme across threshold applying to situations of this general type were first proposed in [17].) We shall call this a variable flavor number scheme (VFNS). We note that our general procedure is completely independent of the choice of renormalization/factorization scale, and that as long as we choose our VFNS coefficient functions such that they satisfy Eq. (3.4) order by order in $\alpha_{s}\left(\mu^{2}\right)$, then correctness is guaranteed. However, we believe that it is sensible to choose the renormalization and factorization scale to be $\mu^{2}=Q^{2}$ in both schemes, for all scales and for both light and heavy quark structure functions. ${ }^{2}$ This very simple choice automatically avoids having different scales for different components of the complete structure function, and means that all mass effects are contained entirely within the coefficient functions. It also agrees with the normal asymptotic choice of $\mu^{2}$ $=Q^{2}$ and removes all problems of $\operatorname{logs}$ of $Q^{2} / \mu^{2}$ (the solution of the evolution equations summing such terms) and $\mu^{2} / M^{2}$, and we are left just with the problems of $\ln \left(Q^{2} / M^{2}\right){ }^{3}$ This choice is expressed explicitly in all our equations from now on, though we will discuss the effect of different choices briefly in Sec. IV. Finally, as already mentioned, if we choose the transition scale as precisely $\mu^{2} \equiv Q^{2}=M^{2}$, then all the logs in $Q^{2} / M^{2}$ disappear, and the matching conditions between the partons in the two schemes in Eq. (2.4) are a simple power series in $\alpha_{s}\left(M^{2}\right)$. Thus, performing the matching at $M^{2}$, and solving order by order in $\alpha_{s}\left(Q^{2}\right)$, as in the strictly massless case, we are guaranteed to sum the logs in $Q^{2} / M^{2}$ correctly at zeroth order in $M^{2} / Q^{2}$. Combining

\footnotetext{
${ }^{2}$ Of course, if we reach sufficiently low $Q^{2}$, then we must introduce some finite renormalization scale in order to have a finite expression for heavy quark photoproduction. Since we only consider $Q^{2}>1 \mathrm{GeV}^{2}$, we do not consider this problem in this paper.

${ }^{3}$ In the asymptotic expressions for the FFNS coefficient functions in [14], this choice leads to significant simplification.
}

with the mass corrected coefficient functions to the appropriate order, we should then get the mass corrected structure functions correctly order by order. Unfortunately, the procedure is not quite as simple as this.

We see that the defining expression for $C_{i a}^{\mathrm{VF}}\left(z, Q^{2} / M^{2}\right)$ is in fact of exactly the same form as Eq. (2.5), except that it is now exact at all $Q^{2}$, rather than having corrections of $\mathcal{O}\left(M^{2} / Q^{2}\right)$, and that this time it is the $n_{f}+1$ flavor coefficient functions, which are the unknowns to be solved in terms of the FFNS coefficient functions and the operator matrix elements, rather than the asymptotic form of the FFNS coefficient functions. However, this leaves us with an ambiguity. The index $a$ runs over the gluon and the light quarks, while $b$ also includes the heavy quark. Hence, while the asymptotic FFNS coefficient functions in Eq. (2.5) were defined uniquely in terms of the light $n_{f}+1$ coefficient functions, solving Eq. (3.3) for the $C_{i a}^{\mathrm{VF}}\left(z, Q^{2} / M^{2}\right)$ in terms of the FFNS coefficient functions does not lead to a unique solution.

In order to demonstrate this, let us write out our equations for the VFNS in full. For the case where the photon couples directly to the heavy quark, $H$, we have two equations:

$$
\begin{aligned}
C_{H g}^{\mathrm{FF}, \mathrm{S}}= & C_{H g}^{\mathrm{VF,S}} \otimes A_{g g, H}^{\mathrm{S}}+n_{f} C_{H q}^{\mathrm{VF}, \mathrm{PS}} \otimes A_{q g, H}^{\mathrm{S}}+\left[C_{H H}^{\mathrm{VF}, \mathrm{NS}}+C_{H H}^{\mathrm{VF}, \mathrm{PS}}\right] \\
& \otimes A_{H g}^{\mathrm{S}}
\end{aligned}
$$

and

$$
\begin{aligned}
C_{H q}^{\mathrm{FF}, \mathrm{S}}= & {\left[C_{H H}^{\mathrm{VF}, \mathrm{NS}}+C_{H H}^{\mathrm{VF}, \mathrm{PS}}\right] \otimes A_{H q}^{\mathrm{PS}}+C_{H q}^{\mathrm{VF}, \mathrm{PS}} \otimes\left[A_{q q, H}^{\mathrm{NS}}+n_{f} A_{q q, H}^{\mathrm{PS}}\right] } \\
& +C_{H g}^{\mathrm{VF}, \mathrm{S}} \otimes A_{g q, H}^{\mathrm{S}},
\end{aligned}
$$

where S, NS, and PS refer to the flavor singlet, nonsinglet, and pure singlet (singlet minus nonsinglet), respectively. In the case where the photon couples directly to a light quark, we have three equations. Denoting the massless $\overline{\text { MS }}$ coefficient functions with $n_{f}$ light flavors by $C_{i a}\left(n_{f}\right)$ and the contributions to the light flavor coefficient functions in the FFNS due to heavy quark generation by $C_{i a}^{\mathrm{FF}}$ we have

$$
\begin{aligned}
& C_{q q}^{\mathrm{NS}}+C_{q q}^{\mathrm{FF}, \mathrm{NS}}=C_{q q}^{\mathrm{VF}, \mathrm{NS}} \otimes A_{q q, H}^{\mathrm{NS}}, \\
& C_{q g}^{\mathrm{S}}+C_{q g}^{\mathrm{FF}, \mathrm{S}}= C_{q q}^{\mathrm{VF}, \mathrm{NS}} \otimes A_{q g, H}^{\mathrm{S}}+C_{q g}^{\mathrm{VF}, \mathrm{S}} \otimes A_{g g, H}^{\mathrm{S}}+n_{f} C_{q q}^{\mathrm{VF}, \mathrm{PS}} \\
& \otimes A_{q g, H}^{\mathrm{S}}+C_{q H}^{\mathrm{VF}, \mathrm{PS}} \otimes A_{H g}^{\mathrm{S}},
\end{aligned}
$$

and

$$
\begin{aligned}
C_{q q}^{\mathrm{PS}}+C_{q q}^{\mathrm{FF}, \mathrm{PS}}= & C_{q q}^{\mathrm{VF}, \mathrm{PS}} \otimes\left[A_{q q, H}^{\mathrm{NS}}+n_{f} A_{q q, H}^{\mathrm{PS}}\right]+C_{q H}^{\mathrm{VF}, \mathrm{PS}} \otimes A_{H q}^{\mathrm{PS}} \\
& +C_{q q}^{\mathrm{VF}, \mathrm{NS}} \otimes A_{q q, H}^{\mathrm{PS}}+C_{q g}^{\mathrm{VF}, \mathrm{S}} \otimes A_{g q, H}^{\mathrm{S}} .
\end{aligned}
$$

These are very similar to the Eq. (2.31)-(2.35) in [15] and, as in those equations, we have implicitly divided all pure singlet quantities coupling to quarks and all singlet quantities coupling to gluons by $n_{f}$. Also, as in these previous equations, it is implicit that all quantities on the left-hand side are expanded in the $n_{f}$-flavor $\overline{\mathrm{MS}}$ coupling constant, while those on the right-hand side are expanded in terms of the $\left(n_{f}\right.$ $+1)$-flavor $\overline{\mathrm{MS}}$ coupling. The relationship between the two couplings was calculated in [18] and corrected in [19]. It is 


$$
\begin{aligned}
\alpha_{s, n_{f}+1}\left(Q^{2}\right)= & \alpha_{s, n_{f}}\left(Q^{2}\right)+\alpha_{s, n_{f}}^{2}\left(Q^{2}\right) \frac{1}{3 \pi} T_{f} \ln \left(Q^{2} / M^{2}\right) \\
& +\alpha_{s, n_{f}}^{3}\left(Q^{2}\right) \frac{1}{\pi^{2}}\left(\frac{1}{9} T_{f}^{2} \ln ^{2}\left(Q^{2} / M^{2}\right)\right. \\
& +\frac{1}{12}\left(5 C_{A} T_{f}+4 C_{f} T_{f}\right) \ln \left(Q^{2} / M^{2}\right) \\
& \left.+\frac{13}{48} T_{f} C_{f}-\frac{2}{9} T_{f} C_{A}\right)+\cdots
\end{aligned}
$$

where the coefficient of the leading log at each order in $\alpha_{s, n_{f}}\left(Q^{2}\right)$ is the same in all schemes, but other coefficients depend on details of renormalization, in particular, whether the mass $M$ is the fixed or running mass. The particular choice above corresponds to a fixed heavy quark mass at NLO.

The difference between our expressions for the coefficient functions and those in [15] is that the coefficient functions on the right-hand side are the VFNS coefficient functions. Not only does this mean that the equations are meant to hold including terms of $\mathcal{O}\left(M^{2} / Q^{2}\right)$, and that we solve for the coefficient functions on the right-hand side, but also that there is a difference between the coefficient functions, which couple to the heavy quark distribution and those coupling to the light quark distributions. For example, while $C_{H H}^{\mathrm{VF}, \mathrm{NS}}$ and $C_{q q}^{\mathrm{VF}, \mathrm{NS}}$ must be identical in the limit $Q^{2} \rightarrow \infty$, they certainly do not have to be identical at moderate $Q^{2}$, and physical intuition suggests they should not be. This means that unlike [15], we do not have five equations for five unknowns, but we have five equations for eight unknowns. In order to reduce to the correct ZM-VFNS at very high $Q^{2}$, we must choose definitions for the mass-corrected coefficient functions, which reduce to the $n_{f}+1$ light parton coefficient functions as $Q^{2} \rightarrow \infty$, but this constraint still leaves a great deal of freedom.

As an example let us consider what is in practice the most important case, the equation for the boson-gluon fusion coefficient function for the heavy quark structure function $F_{2, H}\left(x, Q^{2}\right)$, (3.6). The expansion of $C_{2, H g}^{\mathrm{FF}, \mathrm{S}}$ begins at $\mathcal{O}\left(\alpha_{s}\left(Q^{2}\right)\right)$ as does $C_{2, H g}^{\mathrm{VF}, \mathrm{S}}$ and $A_{H g}^{\mathrm{S}}$, while $A_{g g, H}^{\mathrm{S}}$ and $C_{2, H H}^{\mathrm{VF}, \mathrm{NS}}$ begin at zeroth order. Using the known expressions for the operator matrix elements, we obtain the lowest-order equation relating the FFNS coefficient functions and the VFNS coefficient functions

$$
\begin{aligned}
C_{2, H g}^{\mathrm{FF}, \mathrm{S}, 1}\left(z, Q^{2} / M^{2}\right)= & C_{2, H g}^{\mathrm{VF}, \mathrm{S}, 1}\left(z, Q^{2} / M^{2}\right)+\left(\ln \left(Q^{2} / M^{2}\right)\right. \\
& \left.+c_{\mathrm{rs}}\right) P_{q g}^{0} \otimes C_{2, H H}^{\mathrm{VF}, \mathrm{NS}, 0}\left(Q^{2} / M^{2}\right),
\end{aligned}
$$

where $P_{q g}^{0}(z)$ is the lowest-order splitting function, and $c_{\underline{\mathrm{rs}}}$ is renormalization scheme dependent, but $c_{\mathrm{rs}}=0$ in $\overline{\mathrm{MS}}$ scheme. Hence, we have freedom in how we choose our zeroth-order heavy quark nonsinglet coefficient function, and this then determines our first-order mass-corrected gluon coefficient function. More generally, we have freedom in how we define each of the three coefficient functions coupling to the heavy quark $C_{H H}^{\mathrm{VF}, \mathrm{NS}}, C_{H H}^{\mathrm{VF}, \mathrm{PS}}$, and $C_{q H}^{\mathrm{VF}, \mathrm{PS}}$ at each order in perturbation theory, being constrained only by the requirement that they are of the correct form as $Q^{2} \rightarrow \infty$.

Of course, there cannot truly be an ambiguity in the orderby-order definition of the structure functions. In order to illustrate this, consider the structure function $F_{2}\left(x, Q^{2}\right)$. We also come back to the point concerning renormalization scheme dependence. In order to maintain renormalization scheme consistency, we must be very careful about the way in which we order the expressions. Doing this correctly does not remove the ambiguity in our definitions of the coefficient functions, but it does render this ambiguity physically meaningless, even order by order. Let us consider specifically the heavy quark contribution to the structure function $F_{2}\left(x, Q^{2}\right)$ in the general VFNS. In fact we will discuss its $\ln Q^{2}$ derivative, since it is the evolution of $F_{2, H}\left(x, Q^{2}\right)$ which is a more natural quantity. Taking the $\ln Q^{2}$ derivative of $F_{2, H}\left(x, Q^{2}\right)$ and keeping all terms up to $\mathcal{O}\left(\alpha_{s}\left(Q^{2}\right)\right)$, multiplying the VFNS parton distributions, we obtain

$$
\begin{aligned}
\frac{d F_{2, H}\left(x, Q^{2}\right)}{d \ln \left(Q^{2}\right)}= & \frac{d C_{2, H H}^{\mathrm{VF}, \mathrm{NS}, 0}\left(Q^{2} / M^{2}\right)}{d \ln \left(Q^{2}\right)} \otimes\left(H\left(Q^{2}\right)+\bar{H}\left(Q^{2}\right)\right)_{0} \\
& +\frac{\alpha_{s, n_{f}+1}\left(Q^{2}\right)}{2 \pi} C_{2, H H}^{\mathrm{VF,NS}, 0}\left(Q^{2} / M^{2}\right) \\
& \otimes\left[P_{q g}^{0} \otimes g_{0}^{n_{f}+1}\left(Q^{2}\right)+P_{q q}^{0}\right. \\
& \left.\otimes\left(H\left(Q^{2}\right)+\bar{H}\left(Q^{2}\right)\right)_{0}\right] \\
& +\frac{\alpha_{s, n_{f}+1}\left(Q^{2}\right)}{2 \pi}\left(\frac{d C_{2, H g}^{\mathrm{VF}, 1}\left(Q^{2} / M^{2}\right)}{d \ln \left(Q^{2}\right)}\right. \\
& \otimes g_{0}^{n_{f}+1}\left(Q^{2}\right)+\frac{d C_{2, H H}^{\mathrm{VF}, \mathrm{NS}, 1}\left(Q^{2} / M^{2}\right)}{d \ln \left(Q^{2}\right)} \\
& \left.\otimes\left(H\left(Q^{2}\right)+\bar{H}\left(Q^{2}\right)\right)_{0}\right) .
\end{aligned}
$$

Asymptotically, the second and third term in this expression reduce to the required form for the leading-order expression in the ZM-VFNS. All other terms fall off to zero in this limit, so we are guaranteed the correct asymptotic expression using this prescription. However, at low $Q^{2}$ the exact form of the expression is highly sensitive to our particular choice of coefficient functions. This clearly means that we do not have a truly well-ordered solution and this is because the true ordering of the coefficient functions $C_{2, H b}^{\mathrm{VF}}\left(z, Q^{2} / M^{2}\right)$ is not as simple as just order by order in $\alpha_{s, n_{f}+1}\left(Q^{2}\right)$ due to their dependence on the quark mass. Indeed, their ordering is the crux of the problem, and we will explore this below.

In order to examine the true ordering of our expression, we will express it in terms of unambiguously defined quantities, and also in terms of those, where the ordering is relatively straightforward. Hence we will express it in terms of the FFNS parton distributions, the mass-dependent coefficient functions $C_{2, H b}^{\mathrm{VF}}\left(z, Q^{2} / M^{2}\right)$, the operator matrix elements and the coupling $\alpha_{s, n_{f}}\left(Q^{2}\right)$. The FFNS parton distributions are correctly ordered simply by solving their evolution equations to a given order. The operator matrix 
elements are ordered according to the power of $\alpha_{s, n_{f}+1}\left(Q^{2}\right)$ minus the power of $\ln \left(Q^{2} / M^{2}\right)$, i.e., the leading-order term is of the form

$$
\begin{aligned}
A_{a b}^{0}\left(z, Q^{2} / M^{2}\right)= & \delta_{a b} \delta(1-z)+\sum_{n=1}^{\infty}\left(\frac{\alpha_{s, n_{f}+1}\left(Q^{2}\right)}{2 \pi}\right)^{n} \\
& \times \ln ^{n}\left(Q^{2} / M^{2}\right) a_{n}(z) .
\end{aligned}
$$

The $n_{f}+1$-flavor coupling constant is defined in terms of the $n_{f}$-flavor coupling in an analogous manner, i.e., the leadingorder relation is

$$
\begin{aligned}
& \alpha_{s, n_{f}+1}\left(Q^{2}\right) \\
& =\alpha_{s, n_{f}}\left(Q^{2}\right)+\sum_{n=1}^{\infty} \alpha_{s, n_{f}}^{n+1}\left(Q^{2}\right)\left(\frac{T_{f}}{3 \pi}\right)^{n} \ln ^{n}\left(Q^{2} / M^{2}\right) .
\end{aligned}
$$

First using the expression for $C_{2, H g}^{\mathrm{VF}, 1}\left(z, Q^{2} / M^{2}\right)$ (3.12), but only keeping the leading-order part of the operator matrix element, i.e., leaving out the $c_{\mathrm{rs}}$, and substituting into Eq. (3.13), we obtain

$$
\begin{aligned}
\frac{d F_{2, H}\left(x, Q^{2}\right)}{d \ln \left(Q^{2}\right)}= & \frac{d C_{2, H H}^{\mathrm{VF}, \mathrm{NS}, 0}\left(Q^{2} / M^{2}\right)}{d \ln \left(Q^{2}\right)} \otimes\left(H\left(Q^{2}\right)+\bar{H}\left(Q^{2}\right)\right)_{0} \\
& +\frac{\alpha_{s, n_{f}+1}\left(Q^{2}\right)}{2 \pi} C_{2, H H}^{\mathrm{VF}, \mathrm{NS}, 0}\left(Q^{2} / M^{2}\right) \otimes P_{q q}^{0} \\
& \otimes\left(H\left(Q^{2}\right)+\bar{H}\left(Q^{2}\right)\right)_{0} \\
& +\frac{\alpha_{s, n_{f}+1}\left(Q^{2}\right)}{2 \pi}\left(\frac{d C_{2, H g}^{\mathrm{FF}, 1}\left(Q^{2} / M^{2}\right)}{d \ln \left(Q^{2}\right)}\right. \\
& \left.-\frac{d C_{2, H H}^{\mathrm{VFN}, 0}\left(Q^{2} / M^{2}\right)}{d \ln \left(Q^{2}\right)} \otimes P_{q g}^{0} \ln \left(Q^{2} / M^{2}\right)\right) \\
& \otimes g_{0}^{n_{f}+1}\left(Q^{2}\right)+\frac{\alpha_{s, n_{f}+1}\left(Q^{2}\right)}{2 \pi} \\
& \times \frac{d C_{2, H H}^{\mathrm{VF}, \mathrm{NS}, 1}\left(Q^{2} / M^{2}\right)}{d \ln \left(Q^{2}\right)} \otimes\left(H\left(Q^{2}\right)+\bar{H}\left(Q^{2}\right)\right)_{0} .
\end{aligned}
$$

We can then be more detailed by using the explicit expressions for $\left(H\left(z, Q^{2}\right)+\bar{H}\left(z, Q^{2}\right)\right)_{0}$ and $g_{0}^{n_{f}+1}\left(z, Q^{2}\right)$ in terms of the FFNS parton distributions, i.e.,

$$
\begin{aligned}
\left(H\left(z, Q^{2}\right)+\bar{H}\left(z, Q^{2}\right)\right)_{0}= & \frac{\alpha_{s, n_{f}}\left(Q^{2}\right)}{2 \pi} \ln \left(Q^{2} / M^{2}\right) P_{q g}^{0} \\
& \otimes g_{0}^{n_{f}}\left(Q^{2}\right) \\
& +\mathcal{O}\left(\alpha_{s, n_{f}}^{2}\left(Q^{2}\right) \ln ^{2}\left(Q^{2} / M^{2}\right)\right)
\end{aligned}
$$

and

$$
\begin{aligned}
g_{0}^{n_{f}+1}\left(z, Q^{2}\right)= & g_{0}^{n_{f}}\left(z, Q^{2}\right)-\frac{\alpha_{s, n_{f}}\left(Q^{2}\right)}{6 \pi} \ln \left(Q^{2} / M^{2}\right) g_{0}^{n_{f}}\left(z, Q^{2}\right) \\
& +\mathcal{O}\left(\alpha_{s, n_{f}}^{2}\left(Q^{2}\right) \ln ^{2}\left(Q^{2} / M^{2}\right)\right),
\end{aligned}
$$

and also the expression for the $n_{f}$-flavor coupling, (3.15). Doing this and remembering that $d C_{2, H H}^{\mathrm{VF}, \mathrm{NS}, 1}\left(z, Q^{2} / M^{2}\right) /$ $d \ln \left(Q^{2}\right)=\mathcal{O}\left(M^{2} / Q^{2}\right)$, then we obtain

$$
\begin{aligned}
\frac{d F_{2, H}\left(x, Q^{2}\right)}{d \ln \left(Q^{2}\right)}= & \frac{\alpha_{s, n_{f}}\left(Q^{2}\right)}{2 \pi}\left(\frac{d C_{2, H g}^{\mathrm{FF}, 1}\left(Q^{2} / M^{2}\right)}{d \ln \left(Q^{2}\right)} \otimes g_{0}^{n_{f}}\left(Q^{2}\right)\right. \\
& -\frac{\alpha_{s, n_{f}}\left(Q^{2}\right)}{6 \pi} \ln \left(Q^{2} / M^{2}\right) \frac{d C_{2, H g}^{\mathrm{FF}, 1}\left(Q^{2} / M^{2}\right)}{d \ln \left(Q^{2}\right)} \\
& \otimes g_{0}^{n_{f}}\left(Q^{2}\right)+\frac{\alpha_{s, n_{f}}\left(Q^{2}\right)}{2 \pi} \ln \left(Q^{2} / M^{2}\right) \\
& \times C_{2, H H}^{\mathrm{VF}, \mathrm{SH}, 0}\left(Q^{2} / M^{2}\right) \otimes P_{q q}^{0} \otimes P_{q g}^{0} \otimes g_{0}^{n_{f}}\left(Q^{2}\right) \\
& \left.+\mathcal{O}\left(M^{2} / Q^{2}\right) \cdot \mathcal{O}\left(\alpha_{s, n_{f}}^{2}\left(Q^{2}\right) \ln ^{2}\left(Q^{2} / M^{2}\right)\right)\right) .
\end{aligned}
$$

Hence, as well as asymptotically reducing to the correct leading-order expression, the prescription of keeping all terms up to $\mathcal{O}\left(\alpha_{s, n_{f}+1}\left(Q^{2}\right)\right)$, which multiply the leadingorder (LO) VFNS parton distributions has resulted in a unique $\mathcal{O}\left(\alpha_{s, n_{f}}\left(Q^{2}\right)\right)$ expression for the derivative of the heavy quark coefficient function, which also (and necessarily) has the correct threshold behavior. However, it is clear that the $\mathcal{O}\left(\alpha_{s, n_{f}}^{2}\left(Q^{2}\right) \ln Q^{2} / m^{2}\right)$ expression, while having the correct asymptotic limit, has behavior for $Q^{2} \sim M^{2}$ which is sensitive to our choice of coefficient functions. In particular, the behavior of these terms will not generally respect the threshold in $\hat{W}^{2}$. It is clear that at higher orders in $\alpha_{s, n_{f}}\left(Q^{2}\right) \ln \left(Q^{2} / M^{2}\right)$, while we will obtain the correct asymptotic behavior, our lowish $Q^{2}$ behavior will be dependent on the choice of coefficient functions.

If we were to use the expression for the structure function itself, rather than its derivative, in the VFNS by combining the lowest order in $\alpha_{s, n_{f}+1}\left(Q^{2}\right)$ coefficient function with the lowest-order VFNS parton distributions, i.e.,

$$
F_{2, H}\left(x, Q^{2}\right)=C_{2, H H}^{\mathrm{VF}, \mathrm{NS}, 0}\left(Q^{2} / M^{2}\right) \otimes\left(H\left(Q^{2}\right)+\bar{H}\left(Q^{2}\right)\right)_{0},
$$

then again we would be guaranteed the correct LO expression in the asymptotic limit. However, even the leading term in $\alpha_{s, n_{f}}\left(Q^{2}\right) \ln \left(Q^{2} / M^{2}\right)$ (when expressed in terms of the FFNS parton distributions and operator matrix elements) is now completely dependent on the choice of coefficient function, and there is no requirement to have the correct threshold behavior at all.

It should be no surprise that we have this problem. As mentioned earlier in the FFNS, the coefficient functions to all orders contain renormalization-scheme-independent leading-order contributions. By working in the VFNS, we have managed to extract the asymptotic form of this leading- 
order contribution in a relatively simple manner. However, in order to have the full leading-order expression for the structure functions in the VFNS in the threshold region, we need to extract all the information from the leading-order contribution to the FFNS coefficient functions. In principle, the full LO FFNS expression should contain the leading parts of the coefficient functions at all orders in $\alpha_{s, n_{f}}\left(Q^{2}\right)$, and the LO VFNS should include coefficient functions constructed from the full LO FFNS coefficient functions and the full LO operator matrix elements. Absolutely correct matching between the FFNS and the VFNS at $Q^{2}=M^{2}$ leads to the absolutely correct renormalization scheme consistent description of both of these schemes. Thus, in practice the strictly correct LO VFNS is no simpler than using the strictly correct LO FFNS coefficient functions. This is extremely difficult indeed, and in fact probably impossible, there being no clear unique way in which we subtract out the leading-order, renormalization scheme invariant part of the $\mathcal{O}\left(\alpha_{s, n_{f}}^{n}\left(Q^{2}\right)\right)$ FFNS coefficient function except in the asymptotic limit. Indeed, if we were to proceed further for our above example of $d F_{2, H}\left(x, Q^{2}\right) / d \ln \left(Q^{2}\right)$, we would find that our definition of the LO contribution at $\mathcal{O}\left(\alpha_{s, n_{f}}^{2}\left(Q^{2}\right)\right)$ would rely on being able to extract an unambiguous LO, renormalization scheme independent part out of $d C_{2, H g}^{\mathrm{FF}, 2}\left(z, Q^{2} / M^{2}\right) / d \ln \left(Q^{2}\right)$. Though this is simple in the limit $Q^{2} \rightarrow \infty[14,15]$, there does not seem to be any good prescription for arbitrary $Q^{2}$. Therefore it appears as though the VFNS is only any advantage at all in so much that it gives a definition of the charm parton distribution. There does not seem to be any tractable way to produce a prescription for calculating heavy quark structure functions, which both correctly sums the leading logarithms and which has absolutely correct, unique threshold behavior.

\section{A PRACTICAL VFNS}

Bearing in mind the difficulty, or indeed probable impossibility of producing the unambiguous well-ordered calculation of structure functions, it is our aim to produce a prescription for calculating heavy quark structure functions order by order in $\alpha_{s}\left(Q^{2}\right)$ in such a way that we obtain relatively simple expressions, yet maintain as much accuracy as possible over the whole range of $Q^{2}$. Let us first consider the region of $Q^{2}=M^{2}$ and below. In this case if we work order by order in $\alpha_{s, n_{f}}\left(Q^{2}\right)$ in the FFNS, i.e., define the $n$ th-order expression for the heavy quark structure function by

$$
\begin{aligned}
F_{2, H}^{n}\left(x, Q^{2}\right)= & \sum_{m=0}^{n} \sum_{a}\left(\frac{\alpha_{s, n_{f}}\left(Q^{2}\right)}{2 \pi}\right)^{n-m+1} \\
& \times C_{2, H a}^{\mathrm{FF}, n-m+1}\left(M^{2} / Q^{2}\right) \otimes f_{m, a}^{n_{f}}\left(Q^{2}\right), \\
& n=0 \rightarrow \infty,
\end{aligned}
$$

we know that the strictly leading-order terms we ignore are really an order of $\alpha_{s, n_{f}}\left(Q^{2}\right)$ down on those we keep, with no large $\ln \left(Q^{2} / M^{2}\right)$ enhancement, for these values of $Q^{2}$. Adopting this procedure, when working to $\mathcal{O}\left(\alpha_{s, n_{f}}^{n}\left(Q^{2}\right)\right)$ we have an error of $\mathcal{O}\left(\alpha_{s, n_{f}}^{n+1}\left(Q^{2}\right)\right)$ compared to the (in principle) correct calculation, which is the same size as terms not yet calculated and the same size as the renormalization scheme uncertainty. This seems perfectly satisfactory for this region.

Above $Q^{2}=M^{2}$ we want to order our calculation as in the massless case so that in the asymptotic limit of $Q^{2} \gg M^{2}$, we will obtain correctly ordered expressions. Therefore, we order the calculation by using up to $\mathcal{O}\left(\alpha_{s, n_{f}+1}^{n}\left(Q^{2}\right)\right)$ coefficient functions, when solving the evolution equations using up to $\mathcal{O}\left(\alpha_{s, n_{f}+1}^{n+1}\left(Q^{2}\right)\right)$ anomalous dimensions, as required by renormalization scheme consistency, e.g., the leading-order expression is

$$
F_{2, i}^{0}\left(x, Q^{2}\right)=\sum_{b} C_{2, i b}^{\mathrm{VF}, 0}\left(M^{2} / Q^{2}\right) \otimes f_{0, b}^{n_{f}+1}\left(Q^{2}\right),
$$

the NLO expression is

$$
\begin{aligned}
F_{2, i}^{1}\left(x, Q^{2}\right)= & F_{2, i}^{0}\left(x, Q^{2}\right)+\sum_{b}\left(\frac{\alpha_{s, n_{f}+1}\left(Q^{2}\right)}{2 \pi} C_{2, i b}^{\mathrm{VF}, 1}\left(M^{2} / Q^{2}\right)\right. \\
& \left.\otimes f_{0, b}^{n_{f}+1}\left(Q^{2}\right)+C_{b}^{\mathrm{VF}, 0}\left(M^{2} / Q^{2}\right) \otimes f_{1, b}^{n_{f}+1}\left(Q^{2}\right)\right),
\end{aligned}
$$

etc. We stress that this is not a choice, but a strict requirement of obtaining ordered asymptotic, expressions for the structure function itself or its $\ln \left(Q^{2}\right)$ derivative. Of course, in this region of $Q^{2}$ we now have the ambiguity in the definition of the coefficient functions. Thus, since we are not performing the strictly correct ordering, we have to make a choice for these coefficient functions. We do this by defining them order by order in $\alpha_{s, n_{f}+1}\left(Q^{2}\right)$ using the Eqs. (3.6)(3.10), which guarantee correctness to all orders, and also by using the freedom to choose some coefficient functions, i.e., the three coefficient functions coupling to the heavy quarks, to bring us as close to the really correct calculation as possible.

In perturbation theory it is not really the structure function at a particular value of $Q^{2}$ for which we solve, but the evolution at all $Q^{2}$ in terms of the structure functions at some particular $Q^{2}$. Bearing this in mind it seems sensible to constrain our coefficient functions by making the slope of the structure functions at a given order in $\alpha_{s}\left(Q^{2}\right)$ to be continuous across the transition point. In order to examine this constraint, let us again consider the $\ln Q^{2}$-derivative of $F^{2, H}\left(x, Q^{2}\right)$. Approaching the transition point from below, our prescription gives the lowest order $\alpha_{s, n_{f}}\left(Q^{2}\right)$ expression for the $\ln \left(Q^{2}\right)$ derivative as

$$
\frac{d F_{2, H}\left(x, Q^{2}\right)}{d \ln \left(Q^{2}\right)}=\frac{\alpha_{s, n_{f}}\left(Q^{2}\right)}{2 \pi} \frac{d C_{2, H g}^{\mathrm{FF}, 1}\left(Q^{2} / M^{2}\right)}{d \ln \left(Q^{2}\right)} \otimes g_{0}^{n_{f}}\left(Q^{2}\right) .
$$

Just above $Q^{2}=M^{2}$ the $\ln \left(Q^{2}\right)$ derivative of the LO expression in the VFNS is 


$$
\begin{aligned}
\frac{d F_{2, H}\left(x, Q^{2}\right)}{d \ln \left(Q^{2}\right)}= & \frac{d C_{2, H H}^{\mathrm{VF}, 0 \mathrm{~N}, 0}\left(Q^{2} / M^{2}\right)}{d \ln \left(Q^{2}\right)} \otimes\left(H\left(Q^{2}\right)+\bar{H}\left(Q^{2}\right)\right)_{0} \\
& +\frac{\alpha_{s, n_{f}+1}\left(Q^{2}\right)}{2 \pi} C_{2, H H}^{\mathrm{VF,NS}, 0}\left(Q^{2} / M^{2}\right) \\
& \otimes\left[P_{q g}^{0} \otimes g_{0}^{n_{f}+1}\left(Q^{2}\right)+P_{q q}^{0}\right. \\
& \left.\otimes\left(H\left(Q^{2}\right)+\bar{H}\left(Q^{2}\right)\right)_{0}\right],
\end{aligned}
$$

where at the transition point the coupling at this order is continuous. Also we see that the artificial zeroth-order term in Eq. (4.5) disappears at $Q^{2}=M^{2}$ [it is actually canceled in the complete calculation as seen in Eqs. (3.13)-(3.19)], and it is indeed possible to demand the continuity of the derivative across the transition point. Using the constraint and our simple prescription for constructing the structure function in the two regions, we now have a unique form for the previously ambiguous $C_{2, H H}^{\mathrm{VF}, \mathrm{NS}, 0}\left(z, Q^{2} / M^{2}\right)$. Using the fact that $\left(H\left(z, Q^{2}\right)+\bar{H}\left(z, Q^{2}\right)\right)_{0}=0$ at $Q^{2}=M^{2}$, we immediately obtain

$$
C_{2, H H}^{\mathrm{VF}, \mathrm{NS}, 0}\left(Q^{2} / M^{2}\right) \otimes P_{q g}^{0}=\frac{d C_{2, H g}^{\mathrm{FF}, 1}\left(z, Q^{2} / M^{2}\right)}{d \ln \left(Q^{2}\right)},
$$

at $Q^{2}=M^{2}$, and we define $C_{2, H H}^{\mathrm{VF}, \mathrm{NS}, 0}\left(z, Q^{2} / M^{2}\right)$ by demanding that it satisfy this relationship at all $Q^{2}$. As well as guaranteeing the continuity of the evolution of the structure function, this definition also reduces to the correct form for $Q^{2}$ $\gg M^{2}$, since in this limit $d C_{2, H g}^{\mathrm{FF}, 1}\left(z, Q^{2} / M^{2}\right) / d \ln \left(Q^{2}\right)$ $\rightarrow P_{q g}^{0}(z)$ (as we shall see explicitly in Sec. V). This means that the evolution will clearly reduce to the correct asymptotic form of a delta function in the limit $Q^{2} \rightarrow \infty$. Above $Q^{2}=M^{2}$ terms are not exactly as prescribed by the absolutely correct procedure explained in the last section, but they do explicitly maintain the correct threshold behavior since $d C_{2, H g}^{\mathrm{FF}, 1}\left(z, Q^{2} / M^{2}\right) / d \ln \left(Q^{2}\right)$ is zero for $\hat{W}^{2}<4 M^{2}$. At leading order we have in principle an error of $\mathcal{O}\left(\alpha_{s}^{2}\left(Q^{2}\right)\right)$ at the transition point due to the truncation of the FFNS expansion at $\mathcal{O}\left(\alpha_{s, n_{f}}\left(Q^{2}\right)\right)$ [where this error falls like $\left(M^{2} / Q^{2}\right)$ as we approach the correct asymptotic limit] and an error generated by the evolution, which is zero at the transition point, and grows like $\alpha_{s, n_{f}+1}\left(Q^{2}\right) \ln ^{n}\left(Q^{2} / M^{2}\right)$, but falls like $\left(M^{2} / Q^{2}\right)$ as we evolve up from this point. These errors are quite minimal, always being small compared to the quantity being calculated. From Eq. (3.12) we see that we have also completely defined $C_{2, H g}^{\mathrm{VF}, N S, 1}\left(z, Q^{2} / M^{2}\right)$; i.e., in the $\overline{\mathrm{MS}}$ scheme it is

$$
\begin{aligned}
C_{2, H g}^{\mathrm{VF}, 1}\left(z, Q^{2} / M^{2}\right)= & C_{2, H g}^{\mathrm{FF}, 1}\left(z, Q^{2} / M^{2}\right) \\
& -\ln \left(Q^{2} / M^{2}\right) \frac{d C_{2, H g}^{\mathrm{FF}, 1}\left(z, Q^{2} / M^{2}\right)}{d \ln \left(Q^{2}\right)},
\end{aligned}
$$

though we have not yet made use of this coefficient function. However, we notice that each term in this coefficient function separately has the correct threshold behavior in $\hat{W}^{2}$.
At leading order in this prescription the effect discussed above is the only real complication, i.e., the choice for $C_{2, H H}^{\mathrm{VF}, \mathrm{NS}, 0}\left(z, Q^{2} / M^{2}\right)$ is the only one to make. Above the transition point the evolution equations for the partons are now in terms of $n_{f}+1$ massless quarks, and the coupling constant becomes the $\overline{\mathrm{MS}}$ coupling for $n_{f}+1$ massless flavors. But all parton distributions and all other zeroth-order coefficient functions are continuous across the transition.

Of course, although we have determined the lowest-order derivative of the coefficient functions on both sides of the boundary, we must also discuss the value of the structure function itself at $Q^{2}=M^{2}$. Using the zeroth-order expression (4.2), the vanishing of the charm quark distribution at $Q^{2}$ $=M^{2}$ leads to the charm structure function being zero there. Likewise the fact that at zeroth order in the FFNS the coefficient functions for charm production all vanish leads to the zeroth-order value of $F_{2, H}\left(x, M^{2}\right)$ being zero also. Thus, the two expressions are consistent. However, this is unsatisfactory for two reasons. Firstly, the leading-order (order $\alpha_{s}\left(Q^{2}\right)$ ) derivative of the charm structure function is nonzero both above and below $Q^{2}=M^{2}$, provided $x$ is low enough that we are above the threshold in $W^{2}$. Hence, starting with a value of $F_{2, H}\left(x, M^{2}\right)=0$ would lead to negative values for this structure function for $Q^{2}<M^{2}$. Also, one would naturally expect the $\mathrm{LO}$ expression for a quantity to be a reasonable approximation to the quantity itself. The value of $F_{2, H}\left(x, M^{2}\right)$ is not zero, and so the zeroth-order expression is not a good representation of the true value. These problems come about because of a peculiarity of $F_{2}\left(x, Q^{2}\right)$ already discussed in [20]. In general its value at a given $Q_{0}^{2}$ begins at zeroth order in $\alpha_{s}\left(Q_{0}^{2}\right)$, but the $\mathcal{O}\left(\alpha_{s}\left(Q_{0}^{2}\right)\right)$ term is also really part of the leading-order expression since it is renormalization-scheme independent. In contrast the derivative begins at $\mathcal{O}\left(\alpha_{s}\left(Q^{2}\right)\right)$, and all corrections are renormalization scheme dependent and genuinely higher order. Thus, as argued in [20], the input should contain both the zeroth-order term and the $\mathcal{O}\left(\alpha_{s}\left(Q_{0}^{2}\right)\right)$ term, but the latter should play no part in the evolution.

Adopting this procedure we can now specify our leadingorder expressions for the charm structure function as follows. Below $Q^{2}=M^{2}$ we take the $\mathrm{LO}$ expression to be

$$
F_{2, H}^{\mathrm{FF}, 0}\left(x, Q^{2}\right)=\frac{\alpha_{s, n_{f}}\left(Q^{2}\right)}{2 \pi} C_{2, H g}^{\mathrm{FF}, 1}\left(Q^{2} / M^{2}\right) \otimes g_{0}^{n_{f}}\left(Q^{2}\right),
$$

which is equal to the $\mathcal{O}\left(\alpha_{s}\right)$ value at $Q^{2}=M^{2}$, and incorporates the LO evolution down from this scale (up to small corrections). Above $Q^{2}=M^{2}$ the LO expression is

$$
\begin{aligned}
F_{2, H}^{\mathrm{VF}, 0}\left(x, Q^{2}\right)= & F_{2, H}^{\mathrm{FF}, 0}\left(x, M^{2}\right)+C_{2, H H}^{\mathrm{VF}, 0}\left(Q^{2} / M^{2}\right) \\
& \otimes\left(H\left(Q^{2}\right)+\bar{H}\left(Q^{2}\right)\right)_{0},
\end{aligned}
$$

which (up to the constant term) is of the standard form (4.2), and incorporates the correct $\mathrm{LO}$ evolution. In practice the constant term becomes almost insignificant as soon as $Q^{2}$ $>4 M^{2}$. Now we should consider the NLO expressions.

At NLO the situation is rather more complicated because more terms come into play. We now define FFNS expressions by including terms up to order $\alpha_{s, n_{f}}^{2}\left(Q^{2}\right)$ relative to the 
lowest-order parton distributions. The NLO VFNS expression is defined as in Eq. (4.3) (up to a constant again). At this order the situation becomes more complicated because the pure singlet FFNS coefficient function becomes nonzero as does the contribution due to coefficient functions, where the photon couples to a light quark, but where heavy quarks are generated. Let us examine the NLO expressions for the derivative of the heavy quark structure function. First consider the $\mathcal{O}\left(\alpha_{s, n_{f}}^{2}\left(Q^{2}\right)\right)$ expression for the derivative of the heavy quark structure function in the FFNS. This is

$$
\begin{aligned}
\frac{d F_{2, H}\left(x, Q^{2}\right)}{d \ln \left(Q^{2}\right)}= & \left(\frac{\alpha_{s, n_{f}}\left(Q^{2}\right)}{2 \pi}\right)^{2}\left(-\beta_{n_{f}}^{0} C_{2, H g}^{\mathrm{FF}, 1}\left(Q^{2} / M^{2}\right) \otimes g_{0}^{n_{f}}\left(Q^{2}\right)+C_{2, H g}^{\mathrm{FF}, 1}\left(Q^{2} / M^{2}\right)\right. \\
& \otimes\left(P_{g g}^{0, n_{f}} \otimes g_{0}^{n_{f}}\left(Q^{2}\right)+P_{g q}^{0} \otimes \Sigma_{0}^{n_{f}}\left(Q^{2}\right)\right)+\frac{d C_{2, H g}^{\mathrm{FF}, 2}\left(Q^{2} / M^{2}\right)}{d \ln \left(Q^{2}\right)} \otimes g_{0}^{n_{f}}\left(Q^{2}\right) \\
& \left.+\frac{d C_{2, H q}^{\mathrm{FF}, 2}\left(Q^{2} / M^{2}\right)}{d \ln \left(Q^{2}\right)} \otimes \Sigma_{0}^{n_{f}}\left(Q^{2}\right)\right)+\frac{\alpha_{s, n_{f}}\left(Q^{2}\right)}{2 \pi} \frac{d C_{2, H g}^{\mathrm{FF}, 1}\left(Q^{2} / M^{2}\right)}{d \ln \left(Q^{2}\right)} \otimes g_{1}^{n_{f}}\left(Q^{2}\right),
\end{aligned}
$$

where $\Sigma^{n_{f}}\left(z, Q^{2}\right)$ is the singlet light quark distribution. ${ }^{4}$ In the VFNS the situation is even more complicated. Taking the derivative of the NLO expression, and ignoring those terms already in Eq. (4.5), we obtain

$$
\begin{aligned}
\frac{d F_{2, H}\left(x, Q^{2}\right)}{d \ln \left(Q^{2}\right)}= & \frac{d C_{2, H H}^{\mathrm{VF}, 0}\left(Q^{2}\right)}{d \ln \left(Q^{2}\right)} \otimes\left(H\left(Q^{2}\right)+\bar{H}\left(Q^{2}\right)\right)_{1}+\frac{\alpha_{s, n_{f}+1}\left(Q^{2}\right)}{2 \pi}\left(\frac{d C_{2, H H}^{\mathrm{VF}, 1}\left(Q^{2}\right)}{d \ln \left(Q^{2}\right)} \otimes\left(H\left(Q^{2}\right)+\bar{H}\left(Q^{2}\right)\right)_{0}\right. \\
& \left.+\frac{d C_{2, H g}^{\mathrm{VF}, 1}\left(Q^{2}\right)}{d \ln \left(Q^{2}\right)} \otimes g_{0}^{n_{f}+1}\left(Q^{2}\right)+C_{2, H H}^{\mathrm{VFF}, 0}\left(Q^{2} / M^{2}\right) \otimes\left[P_{q q}^{0} \otimes\left(H\left(Q^{2}\right)+\bar{H}\left(Q^{2}\right)\right)_{1}+P_{q g}^{0} \otimes g_{1}^{n_{f}+1}\left(Q^{2}\right)\right]\right) \\
& +\left(\frac{\alpha_{s, n_{f}+1}\left(Q^{2}\right)}{2 \pi}\right)^{2}\left\{-\beta_{n_{f}}^{0}\left[C_{2, H g}^{\mathrm{VF}, 1}\left(Q^{2} / M^{2}\right) \otimes g_{0}^{n_{f}+1}\left(Q^{2}\right)+C_{2, H H}^{\mathrm{VF}, 1}\left(Q^{2} / M^{2}\right) \otimes\left(H\left(Q^{2}\right)+\bar{H}\left(Q^{2}\right)\right)_{0}\right]\right. \\
& +C_{2, H H}^{\mathrm{VFF}, 1}\left(Q^{2} / M^{2}\right) \otimes\left[P_{q q}^{0} \otimes\left(H\left(Q^{2}\right)+\bar{H}\left(Q^{2}\right)\right)_{0}+P_{q g}^{0} \otimes g_{0}^{n_{f}+1}\left(Q^{2}\right)\right]+C_{2, H g}^{\mathrm{VFF}, 1}\left(Q^{2} / M^{2}\right) \otimes\left(P_{g q}^{0} \otimes \Sigma_{0}^{n_{f}+1}\left(Q^{2}\right)\right. \\
& \left.+P_{g g}^{0, n_{f}+1} \otimes g_{0}^{n_{f}+1}\left(Q^{2}\right)\right)+C_{2, H H}^{\mathrm{VF}, 0}\left(Q^{2} / M^{2}\right) \otimes\left[P_{q q}^{\mathrm{NS}, 1, n_{f}+1} \otimes\left(H\left(Q^{2}\right)+\bar{H}\left(Q^{2}\right)\right)_{0}+P_{q q}^{\mathrm{PS}, 1, n_{f}+1}\right. \\
& \left.\left.\otimes \Sigma_{0}^{n_{f}+1}\left(Q^{2}\right)+P_{q g}^{1, n_{f}+1} \otimes g_{0}^{n_{f}+1}\left(Q^{2}\right)\right]\right\} .
\end{aligned}
$$

These expressions are very difficult to compare in general. However, expressing the four flavor quantities in terms of the three flavor quantities, the two are identical at NLO in $\overline{\mathrm{MS}}$ scheme at $Q^{2}=M^{2}$ (the discontinuities in both the parton distributions and the coupling begin at NNLO). Thus, the heavy parton distributions $\left(H\left(z, Q^{2}\right)+\bar{H}\left(z, Q^{2}\right)\right)_{0}$ and $\left(H\left(z, Q^{2}\right)+\bar{H}\left(z, Q^{2}\right)\right)_{1}$ vanish at this point, and so do many other terms in Eq. (4.11). From the definition of $C_{2, H H}^{\mathrm{VF}, 0}\left(z, Q^{2}\right)$, we can see that the term depending on $g_{1}\left(z, Q^{2}\right)$ is the same in both expressions, and using the Eq. (4.7) we can see that $d C_{2, H g}^{\mathrm{VF}, 1}\left(z, Q^{2}\right) / d \ln \left(Q^{2}\right)=0$ at $Q^{2}$ $=M^{2}$. Also in the combination $-\beta_{n_{f}}^{0} f(z)+P_{g g}^{0, n_{f}} \otimes f$ the flavor dependence cancels between the two terms, so this combination is the same in both expressions at $Q^{2}=M^{2}$.

Thus we have a great deal of simplification, when com-

\footnotetext{
${ }^{4}$ We label $P_{g g}^{0}(z)$ by the number of flavors because it is the only leading-order splitting function which depends on this number. The decrease of this splitting function above a threshold accounts for the fact that there is a new parton distribution, and guarantees overall conservation of momentum in the evolution.
}

paring the two expressions at $Q^{2}=M^{2}$. As in the LO case we can equate the terms coupling to the gluon in the two expressions, i.e.,

$$
\begin{aligned}
\frac{d C_{2, H g}^{\mathrm{FF}, 2}\left(z, Q^{2} / M^{2}\right)}{d \ln \left(Q^{2}\right)}= & C_{2, H H}^{\mathrm{VF}, 1}\left(Q^{2} / M^{2}\right) \otimes P_{q g}^{0}+C_{2, H H}^{\mathrm{VF}, 0}\left(Q^{2} / M^{2}\right) \\
& \otimes P_{q g}^{1, n_{f}+1},
\end{aligned}
$$

at $Q^{2}=M^{2}$, and this serves as a definition of the coefficient function $C_{2, H H}^{\mathrm{VF}, 1}\left(z, Q^{2} / M^{2}\right)$ at this $Q^{2}$. However, unlike the LO case we cannot define the coefficient function at all $Q^{2}$ simply by extending this expression to all $Q^{2}$. This is because it will not result in the correct asymptotic expression for $C_{2, H H}^{\mathrm{VF}, 1}\left(z, Q^{2} / M^{2}\right)$, i.e., $d C_{2, H g}^{\mathrm{FF}, 2}\left(z, Q^{2} / M^{2}\right) / d \ln \left(Q^{2}\right)$ contains a $\ln \left(Q^{2} / M^{2}\right)$ term which must be canceled. It is quite easy to find the generalization of Eq. (4.12), however. If one differentiates both sides of Eq. (3.6), and keeps those terms of $\mathcal{O}\left(\alpha_{s, n_{f}}^{2}\left(Q^{2}\right)\right.$ ) which survive as $Q^{2} \rightarrow \infty$ (all terms of the form $d C_{2, b \alpha}^{\mathrm{VF}}\left(z, Q^{2} / M^{2}\right) / d \ln \left(Q^{2}\right)$ vanish in this limit, since the VFNS coefficient functions tend to constants), then one obtains 


$$
\begin{aligned}
\frac{d C_{2, H g}^{\mathrm{FF}, 2}\left(z, Q^{2} / M^{2}\right)}{d \ln \left(Q^{2}\right)}= & C_{2, H H}^{\mathrm{VF}, \mathrm{NS}, 1}\left(Q^{2} / M^{2}\right) \otimes \frac{d A_{H g}^{1}\left(Q^{2} / M^{2}\right)}{d \ln \left(Q^{2}\right)} \\
& +C_{2, H H}^{\mathrm{VF}, \mathrm{NS}, 0}\left(Q^{2} / M^{2}\right) \otimes \frac{d A_{H g}^{2}\left(Q^{2} / M^{2}\right)}{d \ln \left(Q^{2}\right)} \\
& +\frac{1}{3 \pi} \ln \left(Q^{2} / M^{2}\right) C_{2, H H}^{\mathrm{VF}, 0}\left(Q^{2} / M^{2}\right) \\
& \otimes P_{q g}^{0},
\end{aligned}
$$

where the last term comes about from the difference in the derivatives of the three and four flavor couplings. This expression guarantees the correct asymptotic expression for $C_{2, H H}^{\mathrm{VF}, 1}\left(z, Q^{2} / M^{2}\right)$, while Eq. (4.12) guarantees the continuity of the NLO derivative of $F_{2, H}\left(x, Q^{2}\right)$ in the gluon sector, and hence the definition of $C_{2, H H}^{\mathrm{VF}, 1}\left(z, Q^{2} / M^{2}\right)$ must satisfy Eq. (4.12) at $Q^{2}=M^{2}$ and Eq. (4.13) as $Q^{2} \rightarrow \infty$. In fact, at $Q^{2}$ $=M^{2}$ the two expressions are identical, i.e.,

$$
\frac{d A_{H g}^{1}\left(z, Q^{2} / M^{2}\right)}{d \ln \left(Q^{2}\right)}=P_{q g}^{0}(z)
$$

and

$$
\begin{aligned}
\frac{d A_{H g}^{2}\left(z, Q^{2} / M^{2}\right)}{d \ln \left(Q^{2}\right)}= & \left(P_{q q}^{0} \otimes P_{q g}^{0}+P_{q g}^{0} \otimes P_{g g}^{0, n_{f}+1}\right. \\
& \left.-\beta_{0}^{n_{f}+1} P_{q g}^{0}\right) \ln \left(Q^{2} / M^{2}\right)+P_{q g}^{1, n_{f}+1},
\end{aligned}
$$

and we have the very neat result that Eq. (4.13) is the generalization of Eq. (4.12) for all $Q^{2}$, and $C_{2, H H}^{\mathrm{VF}, 1}\left(z, Q^{2} / M^{2}\right)$ is defined by Eq. (4.13).

The above definition of $C_{2, H H}^{\mathrm{VF}, 1}\left(z, Q^{2} / M^{2}\right)$, when substituted into Eq. (3.6), determines the expression for $C_{2, H g}^{\mathrm{VF}, 2}\left(z, Q^{2} / M^{2}\right)$ which will be used at NNLO. However, we have now used up our single degree of freedom involved with the heavy quark structure function at NLO. Looking at the terms coupling to the singlet quark distribution in the two expressions (4.10) and (4.11), we find that the first contains

$$
C_{2, H g}^{\mathrm{FF}, 1}\left(Q^{2} / M^{2}\right) \otimes P_{g q}^{0}+\frac{d C_{2, H q}^{\mathrm{FF}, 2}\left(z, Q^{2} / M^{2}\right)}{d \ln \left(Q^{2}\right)},
$$

while the second contains

$$
C_{2, H g}^{\mathrm{VF}, 1}\left(Q^{2} / M^{2}\right) \otimes P_{g q}^{0}+C_{2, H H}^{\mathrm{FF}, 0}\left(Q^{2} / M^{2}\right) \otimes P_{q q}^{1, \mathrm{PS}, n_{f}+1} .
$$

There is no degree of freedom in either of these equations, and no reason for them to be equal at $Q^{2}=M^{2}$, and they are not. Indeed there was no further degree of freedom in the relationships (3.6) and (3.7) required of the heavy quark coefficient functions. Up to this order the only one available was for $C_{2, H H}^{\mathrm{VF}}\left(z, Q^{2} / M^{2}\right)$, and this has been determined by imposing the continuity of the evolution of the structure function in the gluon sector. Indeed, looking at Eq. (3.7) at $\mathcal{O}\left(\alpha_{s}^{2}\left(Q^{2}\right)\right)$, we see that we have already determined $C_{2, H q}^{\mathrm{VF}, \mathrm{PS}, 2}\left(z, Q^{2} / M^{2}\right)$, i.e.,

$$
\begin{aligned}
C_{2, H q}^{\mathrm{VF}, \mathrm{PS}, 2}\left(z, Q^{2} / M^{2}\right)= & C_{2, H q}^{\mathrm{FF}, 2}\left(z, Q^{2} / M^{2}\right)-C_{2, H H}^{\mathrm{VF}, 0}\left(Q^{2} / M^{2}\right) \\
& \otimes A_{H q}^{\mathrm{PS}, 2}\left(Q^{2} / M^{2}\right) .
\end{aligned}
$$

Using the framework we have chosen to define the structure functions, this discontinuity in the derivative of the heavy quark structure function in the singlet sector is unavoidable. There are simply not enough degrees of freedom to avoid it. In practice, since the evolution of the heavy quark structure function is driven very largely by the gluon, since this discontinuity begins only at NLO, and since Eqs. (4.16) and (4.17) are not too different at $Q^{2}=M^{2}$ the effect is tiny. Of course, any discontinuity is only an artifact of the manner in which we are forced to do our fixed order calculations, and would disappear if we were to work all orders. In fact one can show that the discontinuity of the derivative in the singlet sector gets formally smaller as one works to higher orders.

So now we have the definition of our NLO expressions for the heavy-quark structure function both above and below threshold. In the FFNS the definition is the simple extension of Eq. (4.8), being just

$$
\begin{aligned}
F_{2, H}^{\mathrm{FF}, 1}\left(x, Q^{2}\right)= & \frac{\alpha_{s, n_{f}}\left(Q^{2}\right)}{2 \pi}\left(C_{2, H g}^{\mathrm{FF}, 1}\left(Q^{2} / M^{2}\right) \otimes g_{0}^{n_{f}}\left(Q^{2}\right)\right. \\
& \left.+C_{2, H g}^{\mathrm{FF}, 1}\left(Q^{2} / M^{2}\right) \otimes g_{1}^{n_{f}}\left(Q^{2}\right)\right) \\
& +\left(\frac{\alpha_{s, n_{f}}\left(Q^{2}\right)}{2 \pi}\right)^{2}\left(C_{2, H g}^{\mathrm{FF}, 2}\left(Q^{2} / M^{2}\right) \otimes g_{0}^{n_{f}}\left(Q^{2}\right)\right. \\
& \left.+C_{2, H q}^{\mathrm{FF}, 2}\left(Q^{2} / M^{2}\right) \otimes \Sigma_{0}^{n_{f}}\left(Q^{2}\right)\right),
\end{aligned}
$$

which is equal to the $\mathcal{O}\left(\alpha_{s}^{2}\right)$ (i.e., NLO) value for the structure function at $Q^{2}=M^{2}$ and incorporates the NLO (i.e., $\mathcal{O}\left(\alpha_{s, n_{f}}^{2}\left(Q^{2}\right)\right)$ evolution down from this scale (up to small corrections). The VFNS NLO expression is

$$
\begin{aligned}
F_{2, H}^{\mathrm{VF}, 1}\left(x, Q^{2}\right)= & \left(\frac{\alpha_{s}\left(M^{2}\right)}{2 \pi}\right)^{2}\left(C_{2, H g}^{\mathrm{FF}, 2}(1) \otimes g_{0}^{n_{f}}\left(M^{2}\right)+C_{2, H q}^{\mathrm{FF}, 2}(1)\right. \\
& \left.\otimes \Sigma_{0}^{n_{f}}\left(M^{2}\right)\right)+C_{2, H H}^{\mathrm{VF}, 0}\left(Q^{2} / M^{2}\right) \\
& \otimes\left(H\left(Q^{2}\right)+\bar{H}\left(Q^{2}\right)\right)_{0}+C_{2, H H}^{\mathrm{VF}, 0}\left(Q^{2} / M^{2}\right) \\
& \otimes\left(H\left(Q^{2}\right)+\bar{H}\left(Q^{2}\right)\right)_{1}+\frac{\alpha_{s, n_{f}+1}\left(Q^{2}\right)}{2 \pi} \\
& \times C_{2, H H}^{\mathrm{VF}, \mathrm{NS}, 1}\left(Q^{2} / M^{2}\right) \otimes\left(H\left(Q^{2}\right)+\bar{H}\left(Q^{2}\right)\right)_{0} \\
& +\frac{\alpha_{s, n_{f}+1}\left(Q^{2}\right)}{2 \pi} C_{2, H g}^{\mathrm{VF}, 1}\left(Q^{2} / M^{2}\right) \otimes g_{0}^{n_{f}+1}\left(Q^{2}\right),
\end{aligned}
$$

which again, up to the constant term, which is the NLO input (the LO part of the input now being included automatically), is of the standard form and incorporates the correct NLO evolution across the transition point.

At this order we have to make some decision about how we treat the light quark sector. The lowest-order contribution the heavy quark makes to a light sector FFNS coefficient 
function is for the nonsinglet coefficient function at $\mathcal{O}\left(\alpha_{s, n_{f}}^{2}\left(Q^{2}\right)\right)$. Thus in the matching conditions between the FFNS coefficient functions and those in the VFNS in the light quark sector, there are no mass-dependent corrections to the VFNS coefficient functions to $\mathcal{O}\left(\alpha_{s, n_{f+1}}\left(Q^{2}\right)\right)$. Hence, the evolution of the light quark coefficient functions above $Q^{2}=M^{2}$ is exactly as in the massless $n_{f}+1$ flavor case. Nevertheless, we must decide on the form of the structure function at $Q^{2}=M^{2}$ and below this transition point. For the heavy quark structure function we have been keeping heavy quark coefficient functions to one order higher in $\alpha_{s}\left(Q^{2}\right)$ in the FFNS than in the VFNS. This has been for the reason that the explicit $\ln \left(Q^{2}\right)$ dependence in the coefficient functions means that they contribute to the $\ln \left(Q^{2}\right)$ derivative of the structure function at effectively one higher order in $\alpha_{s}\left(Q^{2}\right)$ than the VFNS coefficient functions, and also because the lack of the usual zeroth-order coefficient function makes the $\mathcal{O}\left(\alpha_{s, n_{f}}\left(Q^{2}\right)\right)$ coefficient function the LO one, the $\mathcal{O}\left(\alpha_{s, n_{f}}^{2}\left(Q^{2}\right)\right)$ the NLO one, etc. For the light structure functions there is a zeroth-order coefficient function, so the second argument no longer holds. However, the former one still does, i.e., differentiating the expression for the light quark structure function below $Q^{2}=M^{2}$ and keeping terms of order $\alpha_{s, n_{f}}^{2}\left(Q^{2}\right)$ then $d C_{2, q q}^{\mathrm{FF}, \mathrm{NS}, 2}\left(z, Q^{2} / M^{2}\right) / d \ln \left(Q^{2}\right)$ appears in the expression. This contribution accounts for the effect of the heavy quark to the evolution turning on as $Q^{2}$ increases. For this reason we continue to keep the coefficient functions containing heavy quarks to one higher order than those with only light quarks even in the light sector.

For the heavy quark structure function, because we had terms of higher order in $\alpha_{s}\left(Q^{2}\right)$ below $Q^{2}$ than above it, in order to impose continuity of the structure function at $Q^{2}$ $=M^{2}$, we had to put a contribution to the VFNS expression which is constant, and one order in $\alpha_{s}$ higher than the rest of the expression (we also justified this from renormalization scheme consistency). We now have to adopt a similar procedure for the light quark expressions. The NLO expression for the nonsinglet structure function for $Q^{2}<M^{2}$ is

$$
\begin{aligned}
F_{2, q}^{\mathrm{FF}, \mathrm{NS}, 1}\left(x, Q^{2}\right)= & C_{2, q q}^{\mathrm{NS}, n_{f}, 0} \otimes f_{0}^{\mathrm{NS}, n_{f}}\left(Q^{2}\right)+C_{2, q q}^{\mathrm{NS}, n_{f}, 0} \otimes f_{1}^{\mathrm{NS}, n_{f}}\left(Q^{2}\right) \\
& +\frac{\alpha_{s, n_{f}}\left(Q^{2}\right)}{2 \pi} C_{2, q q}^{\mathrm{NS}, n_{f}, 1} \otimes f_{0}^{\mathrm{NS}, n_{f}}\left(Q^{2}\right) \\
& +\left(\frac{\alpha_{s, n_{f}}\left(Q^{2}\right)}{2 \pi}\right)^{2} C_{2, q q}^{\mathrm{FF}, \mathrm{NS}, 2}\left(Q^{2} / M^{2}\right) \\
& \otimes f_{0}^{\mathrm{NS}, n_{f}}\left(Q^{2}\right) .
\end{aligned}
$$

That for $Q^{2} \geqslant M^{2}$ is equal to

$$
\begin{aligned}
F_{2, q}^{\mathrm{VF}, \mathrm{NS}, 1}\left(x, Q^{2}\right)= & C_{2, q q}^{\mathrm{NS}, n_{f}+1,0} \otimes f_{0}^{\mathrm{NS}, n_{f}+1}\left(Q^{2}\right)+C_{2, q q}^{\mathrm{NS}, n_{f}+1,0} \\
& \otimes f_{1}^{\mathrm{NS}, n_{f}+1}\left(Q^{2}\right)+\frac{\alpha_{s, n_{f}+1}\left(Q^{2}\right)}{2 \pi} C_{2, q q}^{\mathrm{NS}, n_{f}+1,1} \\
& \otimes f_{0}^{\mathrm{NS}, n_{f}+1}\left(Q^{2}\right)+\left(\frac{\alpha_{s}\left(M^{2}\right)}{2 \pi}\right)^{2} C_{2, q q}^{\mathrm{FF}, \mathrm{NS}, 2}(1) \\
& \otimes f_{0}^{\mathrm{NS}, n_{f}}\left(M^{2}\right) .
\end{aligned}
$$

In principle both sides should also contain a term $\propto \alpha_{s}^{2}\left(Q_{0}^{2}\right)$ for the genuinely light NLO input, where $Q_{0}^{2}$ is the scale at which the inputs are chosen. Such a term is always ignored, and would be very small. In practice all the $\mathcal{O}\left(\alpha_{s}^{2}\left(Q^{2}\right)\right)$ terms in the above expression are extremely small as well. The $\mathcal{O}\left(\alpha_{s}^{2}\left(Q^{2}\right)\right)$ evolution derived from the above equations is not precisely continuous at $Q^{2}=M^{2}$ due to terms of inverse powers of $Q^{2} / M^{2}$ present in $d C_{2, q q}^{\mathrm{FF}, \mathrm{NS}, 2}\left(z, Q^{2} / M^{2}\right) / d \ln \left(Q^{2}\right)$. This discontinuity will decrease as we go to higher orders, and these mass-dependent terms get absorbed by higher-order mass-dependent VFNS coefficient functions. We note that leaving the $\mathcal{O}\left(\alpha_{s, n_{f}}^{2}\left(Q^{2}\right)\right)$ term out of of Eq. (4.21) would also lead to a discontinuous evolution (actually more so), since the evolution would take account of $n_{f}$ massless flavors below threshold, but $n_{f}+1$ massless flavors above threshold.

Finally at NLO the light quark pure singlet structure functions have no complications due to the heavy quarks at all. The first nonzero FFNS coefficient functions do not appear until $\mathcal{O}\left(\alpha_{s, n_{f}}^{3}\left(Q^{2}\right)\right)$, and so do not contribute to the evolution until NNLO. So at NLO we just use the $n_{f}$ massless flavor expressions below $Q^{2}=M^{2}$ and the $n_{f}+1$ massless flavor expressions above $Q^{2}=M^{2}$. Continuity of both the structure function and its evolution are automatic.

One could in principle work to progressively higher orders, but of course in practice the NNLO splitting functions and the NNLO FFNS coefficient functions are all unknown at present. Nevertheless, we outline the procedure to be adopted at all orders. For the heavy quark structure function there is essentially nothing new as we progress to higher orders. At $n$th nontrivial order we include all FFNS coefficient functions up to order $\alpha_{s, n_{f}}^{n}\left(Q^{2}\right)$, and all VFNS coefficient functions up to order $\alpha_{s, n_{f}+1}^{n-1}\left(Q^{2}\right)$. In the VFNS expression we always include the $\mathcal{O}\left(\alpha_{s}^{n}\left(M^{2}\right)\right)$ term which ensures continuity of the structure function. We determine $C_{2, H H}^{\mathrm{VF}, n-1}\left(z, Q^{2} / M^{2}\right)$ by demanding continuity of the derivative of the structure function at $\mathcal{O}\left(\alpha_{s}^{n}\right)$ in the gluon sector, and this determination predetermines $C_{2, H g}^{\mathrm{VF}, n}\left(z, Q^{2} / M^{2}\right)$ and $C_{2, H q}^{\mathrm{VF}, n}\left(z, Q^{2} / M^{2}\right)$ by using Eqs. (3.6) and (3.7) to $\mathcal{O}\left(\alpha_{s, n_{f}}^{n}\left(Q^{2}\right)\right)$. At $\mathcal{O}\left(\alpha_{s, n_{f}+1}^{2}\left(Q^{2}\right)\right)$ the coefficient function $C_{2, H H}^{\mathrm{VF}, n}\left(z, Q^{2} / M^{2}\right)$ becomes the sum of the nonsinglet and pure singlet coefficient functions. Neither the condition (3.6) nor the continuity of the structure function and its derivatives determine these two contributions separately, so we are free to separate them as we wish, using the condition that each tends to the correct asymptotic limit. It would also be desirable to choose each so that they respect the kinematic threshold.

For the light quark structure function the procedure at higher orders is also straightforward. At $n$th nontrivial order we include all pure light quark contributions to coefficient functions below $Q^{2}=M^{2}$ up to order $\alpha_{s, n_{f}}^{n-1}\left(Q^{2}\right)$; all mass dependent FFNS coefficient functions up to order $\alpha_{s, n_{f}}^{n}\left(Q^{2}\right)$, and all VFNS coefficient functions up to order $\alpha_{s, n_{f}+1}^{n-1}\left(Q^{2}\right)$. In the VFNS expression we always include the $\mathcal{O}\left(\alpha_{s}^{n}\left(M^{2}\right)\right)$ term which ensures continuity of the structure function. Starting with the $\mathcal{O}\left(\alpha_{s, n_{f}+1}^{2}\left(Q^{2}\right)\right)$ coefficient function, we determine $C_{2, q H}^{\mathrm{VF}, n-1}\left(z, Q^{2} / M^{2}\right)$ by demanding continuity of 
TABLE I. Prescription for the order by order in $\alpha_{s}\left(Q^{2}\right)$ determination of the VFNS coefficient functions for $F_{2}\left(x, Q^{2}\right)$.

\begin{tabular}{|c|c|c|}
\hline $\begin{array}{l}\text { Order of } \\
\text { equality }\end{array}$ & Eq. & Coefficient functions determined \\
\hline$\alpha_{s}^{0}\left(Q^{2}\right)$ & $(3.8)$ & $C_{2, q q}^{\mathrm{VF}, \mathrm{NS}, 0}$ \\
\hline \multirow[t]{3}{*}{$\alpha_{s}\left(Q^{2}\right)$} & $(3.6)$ & $\begin{array}{l}C_{2, H H}^{\mathrm{VF}, \mathrm{NS}, 0}\left[\text { by continuity of }\left(d F_{2, H} / d \ln Q^{2}\right)_{M^{2}} \text { at }\right. \\
\left.\mathcal{O}\left(\alpha_{s}\left(Q^{2}\right)\right)\right], C_{2, H g}^{\mathrm{VF}, 1}\end{array}$ \\
\hline & $(3.8)$ & $C_{2, q q}^{\mathrm{VF}, \mathrm{NS}, 1}$ \\
\hline & $(3.9)$ & $C_{2, q g}^{\mathrm{VF}, 1}$ \\
\hline \multirow[t]{5}{*}{$\alpha_{s}^{2}\left(Q^{2}\right)$} & $(3.6)$ & $\begin{array}{l}C_{2, H H}^{\mathrm{VF}, \mathrm{S}, 1}\left[\text { by continuity of }\left(d F_{2, H} / d \ln Q^{2}\right)_{M^{2}} \text { in gluon }\right. \\
\left.\text { sector at } \mathcal{O}\left(\alpha_{s}^{2}\left(Q^{2}\right)\right)\right], C_{2, H g}^{\mathrm{VF}, 2}\end{array}$ \\
\hline & $(3.7)$ & $C_{2, H q}^{\mathrm{VF}, 2}$ \\
\hline & $(3.8)$ & $C_{2, q q}^{\mathrm{VF}, \mathrm{NS}, 2}$ \\
\hline & $(3.9)$ & $C_{2, q g}^{\mathrm{VF}, 2}$ \\
\hline & $(3.10)$ & $C_{2, q q}^{\mathrm{VF}, \mathrm{PS}, 2}$ \\
\hline \multirow[t]{5}{*}{$\alpha_{s}^{3}\left(Q^{2}\right)$} & $(3.6)$ & $\begin{array}{l}C_{2, H H}^{\mathrm{VF}, \mathrm{S}, 2}\left[\text { by continuity of }\left(d F_{2, H} / d \ln Q^{2}\right)_{M^{2}} \text { in gluon }\right. \\
\left.\text { sector at } \mathcal{O}\left(\alpha_{s}^{3}\left(Q^{2}\right)\right)\right], C_{2, H g}^{\mathrm{VF}, 3}\end{array}$ \\
\hline & $(3.7)$ & $C_{2, H q}^{\mathrm{VF}, 3}$ \\
\hline & $(3.8)$ & $C_{2, q q}^{\mathrm{VF}, 3}$ \\
\hline & $(3.9)$ & $\begin{array}{l}C_{2, q H}^{\mathrm{VF}, 2} \text { [by continuity of }\left(d F_{2, i} / d \ln Q^{2}\right)_{M^{2}} \text { in gluon } \\
\left.\text { sector at } \mathcal{O}\left(\alpha_{s}^{3}\left(Q^{2}\right)\right)\right], C_{2, q g}^{\mathrm{VF}, 3}\end{array}$ \\
\hline & $(3.10)$ & $C_{2, q q}^{\mathrm{VF}, \mathrm{PS}, 3}$ \\
\hline$\cdots$ & $\cdots$ & $\cdots$ \\
\hline \multirow[t]{5}{*}{$\alpha_{s}^{n}\left(Q^{2}\right)$} & $(3.6)$ & $\begin{array}{l}C_{2, H H}^{\mathrm{VF}, \mathrm{S} n-1} \text { [by continuity of }\left(d F_{2, H} / d \ln Q^{2}\right)_{M^{2}} \text { in } \\
\left.\text { gluon sector at } \mathcal{O}\left(\alpha_{s}^{n}\left(Q^{2}\right)\right)\right], C_{s, H g}^{\mathrm{VF}, n}\end{array}$ \\
\hline & $(3.7)$ & - \\
\hline & $(3.8)$ & $C_{2, q q}^{\mathrm{VF}, n}$ \\
\hline & $(3.9)$ & $\begin{array}{l}C_{2, q H}^{\mathrm{VF}, n-1}\left[\text { by continuity of }\left(d F_{2, i} / d \ln Q^{2}\right)_{M^{2}} \text { in gluon }\right. \\
\left.\text { sector at } \mathcal{O}\left(\alpha_{s}^{n}\left(Q^{2}\right)\right)\right], C_{2, q g}^{\mathrm{VF}, n}\end{array}$ \\
\hline & $(3.10)$ & $C_{2, q q}^{\mathrm{VF}, \mathrm{PS} n}$ \\
\hline
\end{tabular}

the derivative of the light structure function at $\mathcal{O}\left(\alpha_{s}^{n}\left(Q^{2}\right)\right)$ in the gluon sector, analogously to the heavy quark sector. With this one degree of freedom eliminated in this way, all other VFNS coefficient functions are determined uniquely order by order in $\alpha_{s}$ by Eqs. (3.8)-(3.10), i.e., this determination of $C_{2, q H}^{\mathrm{VF}, n-1}\left(z, Q^{2} / M^{2}\right) \quad$ predetermines $C_{2, q g}^{\mathrm{VF}, n}\left(z, Q^{2} / M^{2}\right)$ and $C_{2, q q}^{\mathrm{VF}, \mathrm{PS}, n}\left(z, Q^{2} / M^{2}\right)$ by using Eqs. (3.9) and (3.10) to $\mathcal{O}\left(\alpha_{s}^{n}\left(Q^{2}\right)\right)$.

Thus, we have completely defined our prescription for calculating order by order for the structure function $F_{2}\left(x, Q^{2}\right)$. We can sum it up in the form of a table. This is shown in Table I. This method uniquely determines all VFNS coefficient functions, and while not leading to absolutely correctly ordered expressions it is a relatively simple prescription for obtaining order by order structure functions which are very similar to the strictly correctly ordered ones, which reduce to the correctly ordered expressions in the asymptotic limit and which order by order are consistent with all physical requirements. All prescriptions which obey Eqs. (3.6)-(3.10) will be correct when summed to all orders, but some ways of choosing the heavy quark coefficient functions will clearly stay closer to the correct ordering than oth- ers. We believe that our prescription is the best available at present, and we see no easy way to improve upon it. We will demonstrate the results using our prescription in the next section, and see that indeed they do seem to work very well.

Let us briefly discuss the effect of choosing a renormalization/factorization scale other than our simple choice of $\mu^{2}=Q^{2}$. As already mentioned our defining equations for the coefficient functions are of exactly the same form, i.e., Eqs. (3.6)-(3.10), but now both the FFNS and VFNS coefficient functions will depend on $M^{2}, Q^{2}$, and $\mu^{2}$. Letting $\mu^{2}=\mu^{2}\left(Q^{2}, M^{2}\right)$, with the requirement that the transition point is at $Q^{2}$ such that $\mu^{2}=M^{2}$ (else the relatively simple matching of parton distributions at $\mu^{2}=M^{2}$ will no longer hold), we can demand that we remove the ambiguity in the VFNS coefficient functions using exactly the same criteria as above, i.e., that the $\ln Q^{2}$ derivatives of the structure function in the gluon sector should be continuous across the transition point. The new scale will lead to different expressions for the derivatives of the structure functions to those above both because the value of $\mu^{2}$ in the terms is different and because the factors of $\left(d \ln \mu^{2} / d \ln Q^{2}\right)_{\mu^{2}=\mu^{2}\left(Q^{2}, M^{2}\right)}$ which implicitly appear when 
differentiating the structure function with respect to $Q^{2}$ are no longer unity. For example, repeating the procedure for the zeroth-order coefficient function as in Eqs. (4.4) and (4.5) will lead to

$$
\begin{aligned}
& C_{2, H H}^{\mathrm{VF}, \mathrm{NS}, 0}\left(Q^{2} / M^{2}\right) \otimes P_{q g}^{0} \otimes\left(\frac{d \ln \mu^{2}\left(Q^{2}, M^{2}\right)}{d \ln Q^{2}}\right) \\
& =\frac{d C_{2, H g}^{\mathrm{FF}, 1}\left(z, Q^{2} / M^{2}\right)}{d \ln \left(Q^{2}\right)},
\end{aligned}
$$

where $C_{2, H g}^{\mathrm{FF}, 1}\left(z, Q^{2} / M^{2}\right)$ is independent of $\mu^{2}$. Hence, the zeroth-order heavy quark coefficient function determined via our procedure is indeed scale dependent, but that its essential property, i.e., the correct threshold behavior is maintained, and that as soon as $\left(d \ln \mu^{2}\left(Q^{2}, M^{2}\right) / d \ln Q^{2}\right)$ tends to one (as it must if $\mu^{2}=Q^{2}$ asymptotically) it tends to our previous result. It is easy to see that these properties will be maintained for all other VFNS coefficient functions, the correct threshold behavior being guaranteed by the fact that it exists in the FFNS coefficient functions. Hence, the exact, order by order form of the coefficient functions in our procedure does depend on the choice of scale, as in all perturbative calculations in QCD, but the procedure can be implemented for a general choice of scale with the essential features being preserved. The further the scale choice is from our simple $\mu^{2}$ $=Q^{2}$, the further will be the deviation from our results at a given order (though, of course, all choices will converge to a common result if we work to high enough order due to the scale independence of the whole structure function). For the rest of this paper we will keep to the simple and physically motivated choice of $\mu^{2}=Q^{2}$.

Before demonstrating the results using our definition of a VFNS, first let us mention another currently available VFNS, the Aivazis, Collins, Olness, and Tung (ACOT) scheme $[21,22,23]$. Although there is currently no all-orders [24], or even NLO definition (for developments see [25], of the ACOT VFNS (which we will denote by ACOT) in print, we believe that the definition of the coefficient functions in this scheme must be equivalent to that in Eq. (3.5), i.e., the VFNS coefficient functions are related to those in the FFNS by the Equations (3.6)-(3.10). Indeed, at what they call LO, the ACOT coefficient functions satisfy Eq. (3.12). However, they determine the expression for $C_{2, H H}^{\mathrm{VF}, \mathrm{NS}, 0}$ from the tree-level diagram for a massive quark scattering from a boson, and for a photon this gives

$$
\begin{gathered}
\hat{C}_{H H}^{\mathrm{VFNS}, 0}\left(z, Q^{2} / M^{2}\right)=z \delta\left(\hat{x}_{0}-z\right)\left(1+\frac{4 M^{2}}{Q^{2}}\right), \\
\hat{x}_{0}=\left(1+\frac{M^{2}}{Q^{2}}\right)^{-1},
\end{gathered}
$$

where the modified argument of the delta function follows from demanding the on-shell condition for the massive quark, and the remaining factor follows from the parton model for the longitudinal structure function, $F_{L}$ $=4 M^{2} / Q^{2}$, which is added to the transverse component to give $F_{2}$. Inserting into Eq. (3.12) for arbitrary $\mu$ then gives the expression for $\hat{C}_{2, H g}^{\mathrm{VF}, \mathrm{S}, 1}\left(z, Q^{2} / \mu^{2}, Q^{2} / M^{2}\right)$. Presumably the ACOT scheme works at higher orders in a similar manner, with the higher-order heavy quark coefficient functions being calculated explicitly [but needing explicit subtraction of divergences in $\left(Q^{2} / M^{2}\right)$ beyond leading order]. However, we note that ACOT do not usually use the scale choice $\mu^{2}$ $=Q^{2}$ as we do. More common is $\mu^{2}=M^{2}+0.5 Q^{2}(1$ $\left.-M^{2} / Q^{2}\right)^{2}$ [23], which grows more slowly than our choice from the same value at $Q^{2}=M^{2}$ and is $\mu^{2}=0.5 Q^{2}$ asymptotically.

ACOT claims a smooth transition from the FFNS at low $Q^{2}$ order by order. Their "LO" expression for the structure function is

$$
\begin{aligned}
F_{2, H}^{\mathrm{LO}}\left(x, Q^{2}\right)= & \hat{C}_{2, H H}^{\mathrm{VF}, \mathrm{NS}, 0}\left(Q^{2} / M^{2}\right) \otimes\left(H\left(\mu^{2}\right)+\bar{H}\left(\mu^{2}\right)\right)_{0} \\
& +\frac{\alpha_{s, n_{f}+1}\left(\mu^{2}\right)}{2 \pi} \hat{C}_{2, H g}^{\mathrm{VF}, \mathrm{S}, 1}\left(Q^{2} / \mu^{2}, Q^{2} / M^{2}\right) \\
& \otimes g_{0}^{n_{f}+1}\left(\mu^{2}\right),
\end{aligned}
$$

where from Eqs. (3.12) and (4.24),

$$
\begin{aligned}
\hat{C}_{2, H g}^{\mathrm{VF}, \mathrm{S}, 1}\left(z, Q^{2} / \mu^{2}, Q^{2} / M^{2}\right) \\
=C_{2, H g}^{\mathrm{FF}, \mathrm{S}, 1}\left(z, Q^{2} / M^{2}\right)-\left(\ln \left(\mu^{2} / M^{2}\right)+c_{\mathrm{rS}}\right) P_{q g}^{0} \otimes z \delta\left(\hat{x}_{0}-z\right) \\
\\
\quad \times\left(1+\frac{4 M^{2}}{Q^{2}}\right) .
\end{aligned}
$$

There are a number of odd features associated with these expressions. Firstly, the "correct"' threshold behavior comes about only from a conspiracy of cancellation. Neither term in Eq. (4.25) respects the physical threshold individually and $\hat{C}_{2, H g}^{\mathrm{VF}, \mathrm{S}, 1}\left(z, Q^{2} / \mu^{2}, Q^{2} / M^{2}\right)$ has a part with a threshold in $\hat{W}^{2}$ and a part going like Eq. (4.24). In fact, since the first term in Eq. (4.25) grows more quickly than the subtraction term in the second term in Eq. (4.25), there will be nonzero (albeit very small) heavy quark structure function for $W^{2}<4 M^{2}$. Once all the necessary cancellation has taken place, the result is very good. This can be seen in Fig. 8 of [23], and also in Fig. 1 which is calculated using the ACOT "LO" prescription, our choice of renormalization scale, and the parton distributions obtained from our best fit (see later for details). There is a smoother transition in Fig. 8 of [23] than in Fig. 1 because their complicated choice of scale leads to $\mu^{2}$ departing slowly from $M^{2}$ and staying well below $Q^{2}$ and hence to the growth of the charm parton distribution being effectively much slower than for the simple $Q^{2}=\mu^{2}$ choice. The effect of the choice of renormalization scale on the speed of departure of the ACOT result from the LO FFNS result can be seen nicely in Fig. 1 of [26].

However, even though the cancellation of terms works well, Eq. (4.25) is at odds with the usual way of defining a LO expression, which usually only involves zeroth coefficient functions convoluted with the parton distributions obtained from the one-loop evolution equations. It is clearly of mixed order, and indeed, part of the expression is in fact renormalization scheme dependent, which is certainly not correct for a LO expression. If we go to $Q^{2} \gg M^{2}$, Eq. (4.26) does not reduce to any fixed order expression in the ZMVFNS. The first term in Eq. (4.26), represented by the dotted line in Fig. 1, becomes the LO expression in the ZM-VFNS, but the second belongs to the NLO expression. One can see in Fig. 1 that the total LO ACOT result is significantly dif- 

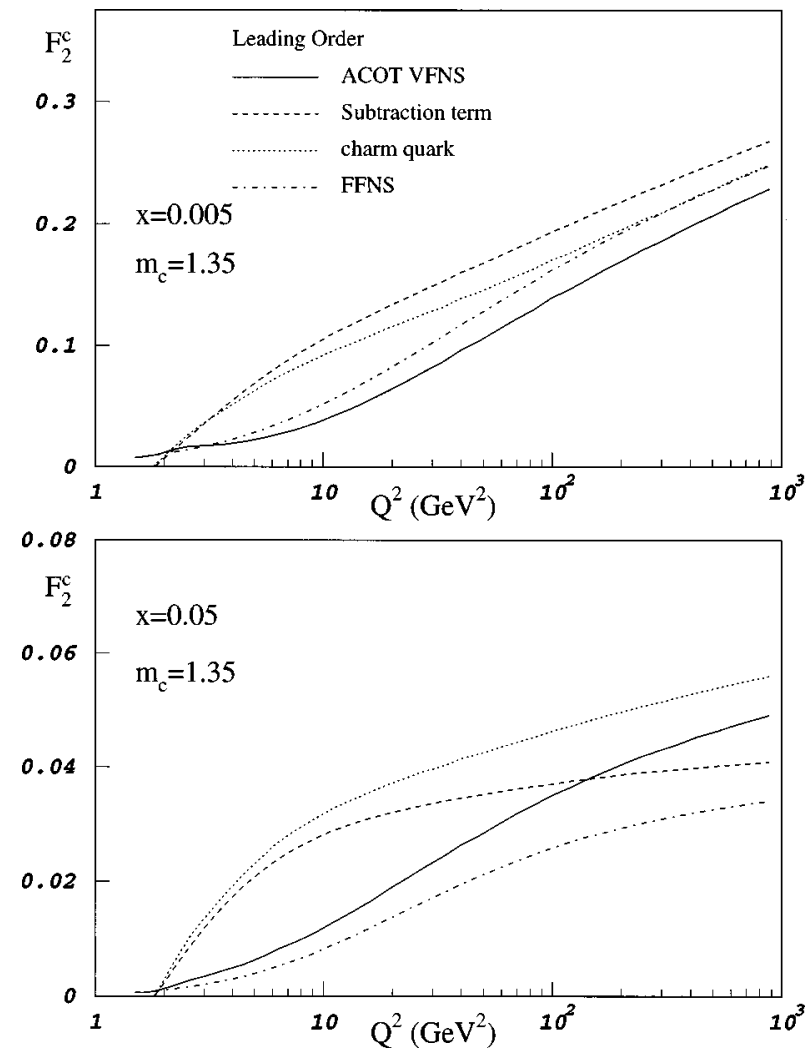

FIG. 1. Charm quark structure function, $F_{2, c}\left(x, Q^{2}\right)$ for $x$ $=0.05$ and $x=0.005$ calculated using the ACOT "LO" prescription, our input parton distributions evolved at LO and renormalization scale $\mu^{2}=Q^{2}$. Shown are the total, the two contributions due to convolution of the coefficient function $C_{2, H g}^{\mathrm{VF}, 1}\left(z, Q^{2} / m_{c}^{2}\right)$ with the gluon distribution (the subtraction term making a negative contribution), and the contribution directly due to the charm quark.

ferent from the asymptotic ZM-VFNS result even at $Q^{2}$ $=1000 \mathrm{GeV}^{2}$. Similarly the derivative of Eq. (4.25) leads to terms both of $\mathcal{O}\left(\alpha_{s, n_{f}+1}\right)$ and $\mathcal{O}\left(\alpha_{s, n_{f}+1}^{2}\right)$, and will have a renormalization scheme dependent part. This mixing of orders is not acceptable.

Alternatively, with the choice of $\hat{C}_{2, H H}^{\mathrm{VF}, \mathrm{NS}, 0}\left(z, Q^{2} / M^{2}\right)$ made, the usual way of ordering the expansion for a structure function leads to serious problems. Using what one would normally consider the LO expression, $F_{2, H}^{0}\left(x, Q^{2}\right)$ $=\hat{C}_{H H}^{\mathrm{VF}, \mathrm{NS}, 0}\left(Q^{2} / M^{2}\right) \otimes\left(H\left(\mu^{2}\right)+\bar{H}\left(\mu^{2}\right)\right)$, has only a sharp threshold in $Q^{2}$ and the rate of growth of $F_{2, H}\left(x, Q^{2}\right)$ would be very discontinuous at $Q^{2}=M^{2}$ and a great deal too fast just above this. This can easily be seen on Fig. 1 where this contribution is represented by the dotted line and labelled "charm quark." It deviates very quickly from both the continuation of the FFNS expression and from the total expression. Using the NLO expression ordered in the usual manner, ${ }^{5}$ the effect would be lessened, but would still be significant. The subtraction piece in $\hat{C}_{2, H g}^{\mathrm{FF}, \mathrm{S}, 1}\left(z, Q^{2} / \mu^{2}, Q^{2} / M^{2}\right)$ would largely cancel the quick growth generated by the $\mathrm{LO}$ evolution of the charm parton

${ }^{5}$ It is an expression of this general form that is used in the recent global fits to data [27].
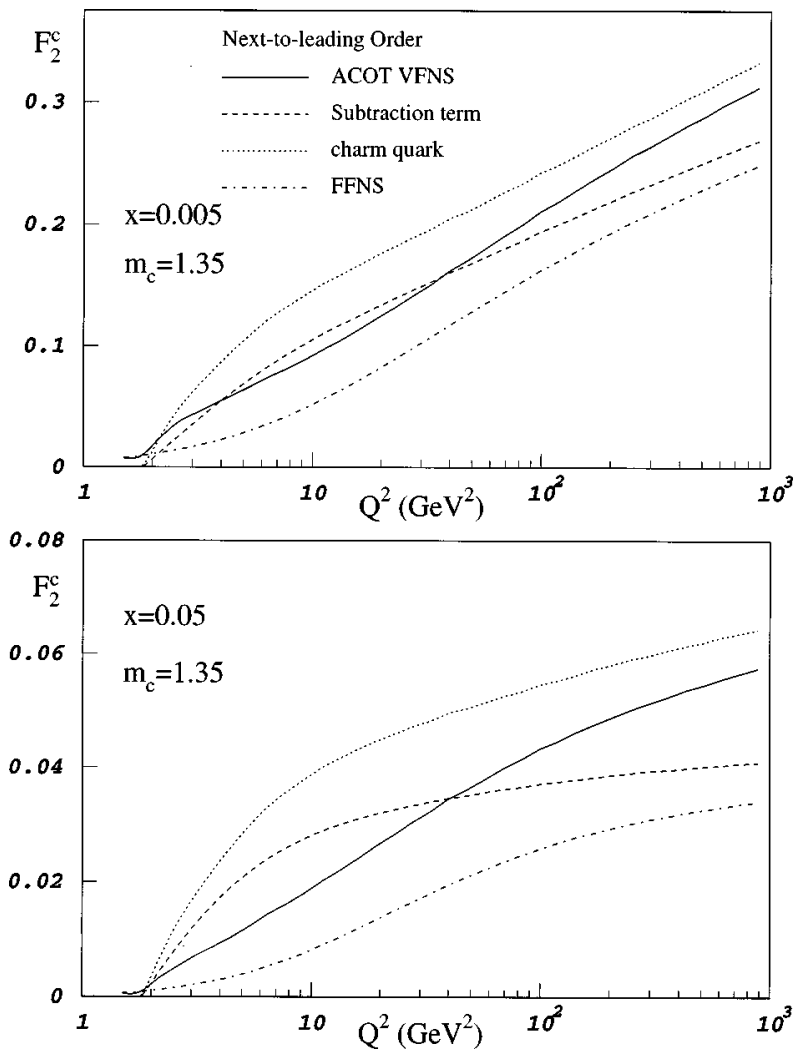

FIG. 2. Same as Fig. 1, but with the partons evolved at NLO.

distribution, but the NLO evolution would still be uncanceled. This effect can be seen in Fig. 9 in [23], where NLO parton distributions are combined with what is called the LO coefficient functions and in Fig. 2, where we do the same thing using our parton distributions and $\mu^{2}=Q^{2}$. Here the subtraction term only partially cancels the charm quark contribution and the total quickly departs from the continuation of the FFNS structure function, and the effect increases at smaller $x$. The all-orders definition of the coefficient functions in Eqs. (3.6)-(3.10), if indeed it is the all orders definition in the ACOT scheme, guarantees that the correct low $Q^{2}$ behavior will be restored when working to all orders, but in this scheme this behavior will come about only due to the mixing of effects at different orders. At low orders the discrepancy is still large. We note that the MRRS scheme [28], which incorporates mass effects into the evolution, but has a similar definition of coefficient functions to ACOT (though with the usual ordering), suffers badly from this problem outlined above. At the transition point, where the heavy quark starts contributing to the heavy quark coefficient function directly, there is a very distinct kink, and the total rises very quickly above the continuation of the FFNS expression, as seen in Figs. 6 and 7 of their paper.

We do not believe that the method used by ACOT (or MRRS) is a satisfactory way in which to define the coefficient functions in a VFNS, and we certainly do not believe that it is unique. It is a choice, as our prescription is a choice, and as we have discussed in Sec. III, we do not believe that any are strictly ' "correct.' However, using the ACOT choice the calculation of the heavy quark coefficient functions proceeds as though the heavy quark parton distribution is due to intrinsic presence of the heavy quark rather than it being 
generated from (at least mainly) the gluon. In particular the heavy quark coefficient function contains no reference to the kinematic threshold in $\hat{W}^{2}$. This necessitates a mixing of orders to get satisfactory results. We believe it is far more useful to choose the heavy quark coefficient functions so that they reflect the physics and all automatically contain at least the correct form of low $Q^{2}$ behavior, and our prescription guarantees this.

\section{THE VFNS IN PRACTICE}

We now discuss how our procedure is implemented in practice. Of course, in practice the first heavy quark we encounter is the charm quark with $m_{c} \approx 1.5 \mathrm{GeV}$. First we consider the LO expression. Denoting $\epsilon=m_{c}^{2} / Q^{2}$ the LO FFNS heavy quark to gluon coefficient function is

$$
\begin{aligned}
C_{2, c g}^{\mathrm{FF}, 1}(z, \epsilon)= & \left(P_{q g}^{0}(z)+4 \epsilon z(1-3 z)-8 \epsilon^{2} z^{2}\right) \ln \left(\frac{1+v}{1-v}\right) \\
& +(8 z(1-z)-1-4 \epsilon z(1-z)) v] \theta\left(\hat{W}^{2}-4 m_{c}^{2}\right),
\end{aligned}
$$

where $\hat{W}^{2}=Q^{2}(1 / z-1)$, the gluon quark center of mass energy, $v$ is the velocity of the heavy quark or antiquark in the photon-gluon center-of-mass frame, defined by $v^{2}=1$ $-4 m_{c}^{2} / \hat{W}^{2}$, and $P_{q g}^{0}(z)=\left(z^{2}+(1-z)^{2}\right)$. These $v$-dependent terms ensure that the coefficient function tends to zero smoothly as $\hat{W}^{2}=4 m_{c}^{2}$ is approached from below, and hence the structure function has a smooth threshold in $W^{2}$. Taking the $\ln Q^{2}$ derivative of this is a straightforward matter and results in

$$
\begin{aligned}
\frac{d C_{2, c g}^{\mathrm{FF}, 1}(z, \epsilon)}{d \ln Q^{2}}= & {\left[\left(P_{q g}^{0}(z)+2 \epsilon \frac{z\left(1-2 z^{2}\right)}{1-z}-16 \epsilon^{2} z^{2}\right) \frac{1}{v}\right.} \\
& +\left[-4 \epsilon z(1-3 z)+16 \epsilon^{2} z^{2}\right] \ln \left(\frac{1+v}{1-v}\right) \\
& +[4 \epsilon z(1-z)] v] \theta\left(\hat{W}^{2}-4 m_{c}^{2}\right),
\end{aligned}
$$

and it is easy to see that in the limit $Q^{2} \rightarrow \infty$,

$$
\frac{d C_{2, c g}^{\mathrm{FF}, 1}(z, \epsilon)}{d \ln Q^{2}} \rightarrow P_{q g}^{0}(z) .
$$

Hence, from Eq. (4.6), we see that $C_{2, c c}^{\mathrm{FF}, \mathrm{NS}, 0}(z, \epsilon)$ must indeed tend to the simple form $z \delta(1-z)$ in this limit.

Solving Eq. (4.6) for $C_{2, c c}^{\mathrm{FF}, \mathrm{NS}, 0}(z, \epsilon)$ at arbitrary $\epsilon$ is not too complicated. Taking moments of both sides the Mellin transformation of $C_{2, c c}^{\mathrm{FF}, \mathrm{NS}, 0}(z, \epsilon)$ is the product of the Mellin transformation of $d C_{2, c g}^{\mathrm{FF}, 1}(z, \epsilon) / d \ln Q^{2}$ and the inverse of the Mellin transformation of $\left(P_{q g}^{0}(z)\right)$, which is

$$
\int_{0}^{1} z^{n-1} P_{q g}^{0}(z) d z=\frac{n^{2}+n+2}{n(n+1)(n+2)} .
$$

This leads to the following expression for the convolution of $C_{2, c c}^{\mathrm{FF}, \mathrm{NS}, 0}(z, \epsilon)$ with the heavy quark distribution,

$$
\begin{aligned}
C_{2, c c}^{\mathrm{FF}, \mathrm{NS}, 0}(\boldsymbol{\epsilon}) \otimes\left(c\left(Q^{2}\right)+\bar{c}\left(Q^{2}\right)\right)= & -\int_{x}^{x_{0}} d z \frac{d C_{2, c g}^{\mathrm{FF}, 1}(z, \boldsymbol{\epsilon})}{d \ln Q^{2}}\left(\frac{x}{z}\right)^{2} \frac{d\left[c\left(x / z, Q^{2}\right)+\bar{c}\left(x / z, Q^{2}\right)\right]}{d(x / z)}+3 \int_{x}^{x_{0}} d x \frac{d C_{2, c g}^{\mathrm{FF}, 1}(z, \boldsymbol{\epsilon})}{d \ln Q^{2}}\left(\frac{x}{z}\right) \\
& \times\left[c\left(x / z, Q^{2}\right)+\bar{c}\left(x / z, Q^{2}\right)\right]-2 \int_{x}^{x_{0}} d z \frac{d C_{2, c g}^{\mathrm{FF}, 1}(z, \epsilon)}{d \ln Q^{2}} \\
& \times \int_{x / z}^{1} d z^{\prime} r\left(z^{\prime}\right) \frac{x}{z z^{\prime}}\left[c\left(x / z z^{\prime}, Q^{2}\right)+\bar{c}\left(x / z z^{\prime}, Q^{2}\right)\right]
\end{aligned}
$$

where $x_{0}=(1+4 \epsilon)^{-1}$ and $r(z)$ is given by

$$
r(z)=z^{1 / 2}\left[\cos \left(\frac{\sqrt{7}}{2} \ln \frac{1}{z}\right)+\frac{3}{\sqrt{7}} \sin \left(\frac{\sqrt{7}}{2} \ln \frac{1}{z}\right)\right]
$$

Using this expression we are able to calculate the LO contribution to the heavy quark structure function using a particular set of parton distributions. In practice we use those obtained from a global fit to structure function data using the NLO formalism (details later). In order to get the LO parton distributions, we simply take the same input parameterizations for the partons, but evolve them using the LO evolution equations. Our prescription for the LO $\alpha_{s}\left(Q^{2}\right)$ across the charm threshold is to define

$$
\alpha_{s}\left(Q^{2}, n\right)=\frac{\beta_{0}^{n}}{4 \pi \ln \left(Q^{2} / \Lambda_{\mathrm{QCD}}^{2}\right)},
$$

and

$$
\alpha_{s, 4}\left(Q^{2}\right)=\alpha_{s}\left(Q^{2}, 4\right),
$$

i.e., $\Lambda_{\mathrm{QCD}}$ is defined for four flavors, and take for three flavors

$$
\alpha_{s, 3}^{-1}\left(Q^{2}\right)=\alpha_{s}^{-1}\left(Q^{2}, 3\right)+\alpha_{s}^{-1}\left(m_{c}^{2}, 4\right)-\alpha_{s}^{-1}\left(m_{c}^{2}, 3\right) .
$$

This prescription precisely reproduces the results of summing the leading logs in $\left(Q^{2} / m_{c}^{2}\right)$ in Eq. (3.15). The results of the LO contribution for the heavy quark coefficient func- 

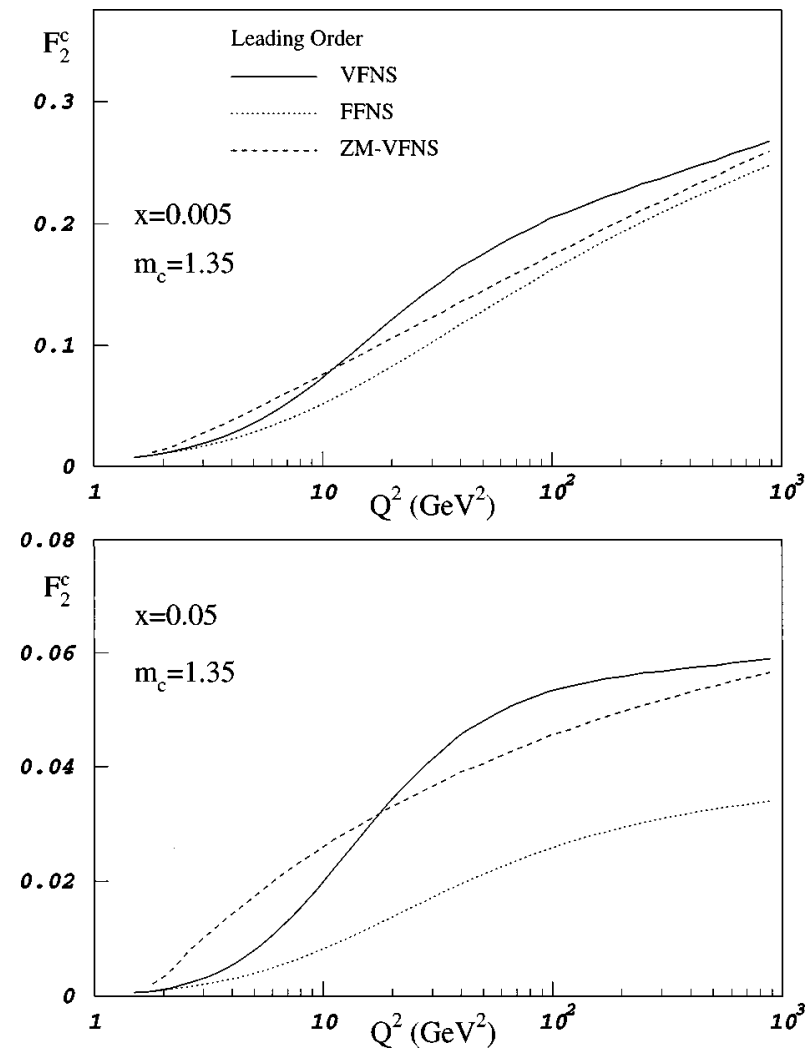

FIG. 3. Charm quark structure function, $F_{2, c}\left(x, Q^{2}\right)$ for $x$ $=0.05$ and $x=0.005$ calculated using our LO prescription, our input parton distributions evolved at LO and renormalization scale $\mu^{2}=Q^{2}$. Also shown are the continuation of the LO FFNS expression and the ZM-VFNS expression both calculated using the same parton distributions and same choice of scale.

tion are shown in Fig. 3, along with the continuation of the LO FFNS expression and also the LO ZM-VFNS expression. One can see that the LO VFNS expression departs very smoothly from the continuation of the LO FFNS expression, then rises above it, and in the limit of very high $Q^{2}$ becomes essentially identical to the LO ZM-VFNS result. This is precisely the behavior we would expect. We also note that unlike other approaches, the expression does not rely on any cancellation between terms.

We now consider also the NLO expression for the heavy quark structure function. As well as the LO coefficient function just introduced, we include the $\mathcal{O}\left(\alpha_{s, n_{f}+1}\left(Q^{2}\right)\right)$ coefficient functions. The expression for $C_{2, c g}^{\mathrm{VF}, 1}(z, \epsilon)$ is as in Eq. (4.7), and is in terms of quantities we have written explicitly above in Eqs. (5.1) and (5.2), i.e.,

$$
C_{2, c g}^{\mathrm{VF}, 1}(z, \epsilon)=C_{2, c g}^{\mathrm{FF}, 1}(z, \epsilon)-\ln \left(Q^{2} / m_{c}^{2}\right) \frac{d C_{2, c g}^{\mathrm{FF}, 1}(z, \epsilon)}{d \ln \left(Q^{2}\right)}
$$

Hence, there are no new problems in implementing $C_{2, c g}^{\mathrm{VF}, 1}(z, \epsilon)$. In the limit $Q^{2} \rightarrow \infty$ the first of these becomes

$$
C_{2, c g}^{\mathrm{FF}, 1}(z, \epsilon) \rightarrow P_{q g}^{0}(z) \ln \left(\frac{(1-z) \epsilon}{z}\right)+[8 z(1-z)-1] .
$$

Using this, along with Eq. (5.3) and the definition (5.10), we see that in the limit $\epsilon \rightarrow \infty$

$$
\begin{aligned}
C_{2, c g}^{\mathrm{VF}, 1}(z, \epsilon) & \rightarrow P_{q g}^{0}(z) \ln \left(\frac{1-z}{z}\right)+[8 z(1-z)-1] \\
& =C_{2, q g}^{n_{f}+1,1}(z),
\end{aligned}
$$

in the $\overline{\mathrm{MS}}$ renormalization scheme.

From the arguments leading up to Eq. (4.12), it is clear that $C_{2, c c}^{\mathrm{VF}, \mathrm{NS}, 1}(z, \epsilon)$ also tends to the correct asymptotic limit, and indeed, all coefficient function are constructed so that this will be true. However, it is not possible to exhibit this in such an explicit manner since the expression for $C_{2, c c}^{\mathrm{VF}, \mathrm{NS}, 1}(z, \epsilon)$ depends on $C_{2, c g}^{\mathrm{FF}, 2}(z, \epsilon)$ for which there is no analytic expression. ${ }^{6}$ Likewise, it would be extremely difficult to implement $C_{2, c c}^{\mathrm{VF}, \mathrm{NS}, 1}(z, \epsilon)$ into the calculation precisely. In practice we find that the contribution to the total heavy quark structure function of this term convoluted with the heavy quark parton distribution is only a very small fraction of the total. Hence, we include this contribution to the total heavy quark structure function in an approximate manner, being confident that it is very far from being significant at the present level of accuracy required.

Using our NLO prescription we use our NLO partons to calculate the heavy quark structure function. Our prescription for the NLO $\alpha_{s}\left(Q^{2}\right)$ is to define $\alpha_{s}\left(Q^{2}, n\right)$ by the standard two loop extension of Eq. (5.7), and then to use Eqs. (5.8) and (5.9) once again. Equation (5.9) does not sum all leading and next-to-leading logs in $\left(Q^{2} / m_{c}^{2}\right)$ absolutely correctly, but is an extremely accurate representation of the precise expression. The NLO charm structure function is shown in Fig. 4 along with the continuation of the NLO FFNS expression and the NLO ZM-VFNS result. As at LO the VFNS departs very smoothly from the continuation of the FFNS expression. Although at this order we have not been able to demand absolute continuity of the derivative of the structure function across $Q^{2}=m_{c}^{2}$, we see that there is no visible evidence of discontinuity at all. In fact the transition from one scheme to the other is smoother than at LO. Also the VFNS stays closer to the continuation of the FFNS at higher $Q^{2}$ at this order. This is as we would expect, since as one works to higher orders in $\alpha_{s}$ in the FFNS, one automatically includes more $\ln \left(Q^{2} / m_{c}^{2}\right)$ terms which are completely summed in the VFNS. At all orders the two schemes become identical insofar as physical quantities are concerned. At very high $Q^{2}$ then our expression tends towards the NLO ZM-VFNS exactly as required.

Of course, at higher $Q^{2}$ we also have effects due to the bottom quark which has $m_{b} \approx 5 \mathrm{GeV}$. Below $Q^{2}=m_{b}^{2}$ there is no bottom quark distribution and we take account of the bottom quark effects by using treating all diagrams including bottom quarks in the fixed flavor scheme, and all other effects decouple. At $Q^{2}=m_{b}^{2}$ we switch to a VFNS for inclu-

\footnotetext{
${ }^{6}$ We are grateful to Jack Smith and Steve Riemersma for providing the extensive program to compute the $\mathcal{O}\left(\alpha_{s}^{2}\right)$ FFNS coefficient functions [29].
} 

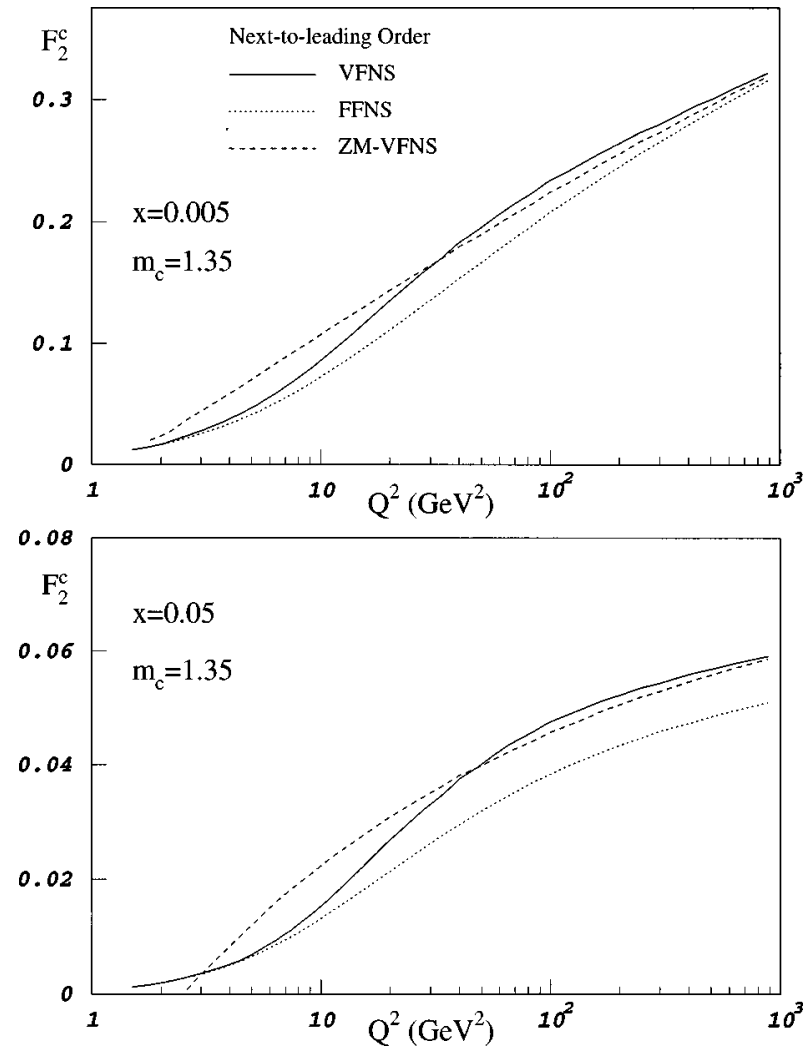

FIG. 4. Same as Fig. 3, but with NLO prescriptions and NLO parton distributions.

sion of the bottom quark effects. Analogously to the charm quark this involves switching to a 5 flavor coupling constant, defined by

$$
\alpha_{s, 5}^{-1}\left(Q^{2}\right)=\alpha_{s}^{-1}\left(Q^{2}, 5\right)+\alpha_{s}^{-1}\left(m_{b}^{2}, 4\right)-\alpha_{s}^{-1}\left(m_{b}^{2}, 5\right),
$$

and beginning the evolution of the bottom quark density. The VFNS coefficient functions are defined using a generalization of Eqs. (3.6)-(3.10). There are now two heavy quarks in the definition of the fixed flavor number scheme, so we have two extra equations for $C_{2, b g}^{\mathrm{FF}, \mathrm{S}}$ and $C_{2, b q}^{\mathrm{FF}, \mathrm{S}}$, and there are now extra VFNS coefficients such as $C_{2, b g}^{\mathrm{VF}, \mathrm{S}}$ or $C_{2, b c}^{\mathrm{FF}, \mathrm{PS}}$. Also, the finite operator matrix elements will depend on both the charm and bottom mass effects. However, exactly the same principles as outlined in the last section apply for determining the VFNS coefficient functions. At low orders in $\alpha_{s}\left(Q^{2}\right)$ there is no mixing between the charm mass effects and the bottom mass effects. Hence, the VFNS charm coefficients functions we have mentioned explicitly above remain the same above $Q^{2}=m_{b}^{2}$ (except for a completely negligible change in $C_{2, c c}^{\mathrm{FF}, \mathrm{NS}, 1}$ ) and the bottom quark coefficient functions are obtained simply by replacing $m_{c}$ with $m_{b}$ and $n_{f}$ +1 -flavor splitting functions with $n_{f}+2$-flavor splitting functions. At higher orders the VFNS charm coefficient functions change above $Q^{2}=m_{b}^{2}$, acquiring $m_{b}$ dependent corrections (in particular $C_{2, c b}^{\mathrm{FF}, \mathrm{PS}}$ comes into existence), and bottom coefficient functions acquire charm mass corrections.

Thus, we have described how one may implement our prescription for the VFNS in practice, showing that there is
TABLE II. Comparison of quality of fits for a wide variety of structure function data $[2,3,33,39-41]$ using our prescription for heavy quarks at NLO (TR) and the NLO fits MRRS and $\operatorname{MRS}\left(R_{2}\right)$ and a NLO fit using the FFNS. We do not include the small- $x$, low$Q^{2}$ data in the second of [2] in our fit in order to make a direct comparison with the previous fits.

\begin{tabular}{|c|c|c|c|c|c|}
\hline \multirow[b]{2}{*}{ Experiment } & \multirow{2}{*}{$\begin{array}{c}\text { Data } \\
\text { points }\end{array}$} & \multicolumn{4}{|c|}{$\chi^{2}$} \\
\hline & & TR & MRRS & $\operatorname{MRS}\left(R_{2}\right)$ & FFNS \\
\hline $\mathrm{H} 1 F_{2}^{e p}$ & 193 & 135 & 133 & 149 & 147 \\
\hline ZEUS $F_{2}^{e p}$ & 204 & 274 & 290 & 308 & 310 \\
\hline BCDMS $F_{2}^{\mu p}$ & 174 & 262 & 271 & 320 & 291 \\
\hline $\mathrm{NMC} F_{2}^{\mu p}$ & 130 & 144 & 145 & 135 & 158 \\
\hline $\mathrm{NMC} F_{2}^{\mu d}$ & 130 & 112 & 119 & 99 & 130 \\
\hline $\mathrm{E} 665 F_{2}^{\mu p}$ & 53 & 61 & 60 & 62 & 61 \\
\hline $\mathrm{E} 665 F_{2}^{\mu d}$ & 53 & 53 & 54 & 60 & 51 \\
\hline SLAC $F_{2}^{e p}$ & 70 & 98 & 96 & 95 & 99 \\
\hline
\end{tabular}

no real difficulty. We have also demonstrated that the results have precisely the properties that our theoretical arguments in the previous section lead us to expect. In order to make even more concrete statements regarding the suitability of our VFNS for the calculation of structure functions, we will now discuss a comparison with data.

\section{PHENOMENOLOGICAL RESULTS}

Using the prescription for heavy partons discussed above, we can calculate the full structure functions in terms of input parton densities for the light quarks and gluon. The input scale is chosen as $Q_{0}^{2}=1 \mathrm{GeV}^{2}$, and the input parton distributions are then determined by performing a best fit to a wide variety of structure function and related data. Hence we repeat the type of procedure adopted by MRS and CTEQ (and others) in their global determination of parton distributions. We note that apart from the masses $m_{c}$ and $m_{b}$, which we fix at $1.35 \mathrm{GeV}$ and $4.3 \mathrm{GeV}$, respectively, the heavy quark contributions to structure functions have no free parameters. The overall description of the data is shown in Table II. ${ }^{7}$ We compare the quality of the fit with that obtained using the FFNS approach, ${ }^{8}$ the MRS $\left(\mathrm{R}_{2}\right)$ fit [30], which uses the ZM-VFNS prescription with a phenomenologically motivated smoothing function and an alternative VFNS, the Martin-Roberts-Ryskin-Stirling (MRRS) approach. There is a clear improvement when compared to the FFNS for all experiments where charm makes any real con-

\footnotetext{
${ }^{7}$ We note that we do not alter the values of $F_{2}\left(x, Q^{2}\right)$ for the HERA data to take account of our predictions for $F_{L}\left(x, Q^{2}\right)$, as should really be done. The $F_{L}\left(x, Q^{2}\right)$ values used in [2] and [3] are obtained using a NLO-in- $\alpha_{s}\left(Q^{2}\right)$ calculation, and so are not very different from ours in general, and the number of points affected is relatively small. Hence the quality of the overall fit is very insensitive to the neglect of this small correction.

${ }^{8}$ Because of the additional computing time required, this fit is not as exhaustive as the others. Nevertheless, we are convinced that it is extremely close to the best possible fit.
} 
tribution to the structure function, a large improvement compared to the MRS $\left(\mathrm{R}_{2}\right)$ fit for the HERA and BCDMS data, though a slight worsening for New Muon Collaboration (NMC) data, and a small, but definite improvement, when compared to the Martin-Roberts-Ryskin-Stirling (MRRS) fit. ${ }^{9}$ We can understand these features as follows. It is general problem for the NLO fit to generate large enough $d F_{2}\left(x, Q^{2}\right) / d \ln Q^{2}$ for smallish $x$ and high $Q^{2}$ in all schemes [a problem cured by correct inclusion of leading $\ln (1 / x)$ terms]. However, this problem is worst in the FFNS, since the rate of growth of charm is smaller at high $Q^{2}$, than in the other schemes as seen in Fig. 4. Hence, the fit using the FFNS is worse for the HERA and NMC data than the VFNS fit. We can understand the improvement of the VFNS fit over the ZM-VFNS for the HERA data by the fact that much of the data lies near the threshold region, and the shape of the structure function predicted by the ZM-VFNS is incorrect in this region, i.e., the slope of $d F_{2}\left(x, Q^{2}\right) / d \ln Q^{2}$ changes abruptly at $Q^{2}=m_{c}^{2}$ rather than smoothly over a wide range of $Q^{2}$. However, we note that the artificially large charm at $x \sim 0.04$ and $Q^{2} \sim 15 \mathrm{GeV}^{2}$ in the ZM-VFNS actually helps the ZM-VFNS fit to NMC data slightly. As far as the BCDMS data is concerned, charm only comprises a very small proportion of the total structure function for this data, but the errors on the data are extremely small, so producing the correct form of charm will have some effect on the fit. The fact that the VFNS does give the best fit is some indication of its appropriateness. The small, but definite improvement over the MRRS approach also adds support to our particular VFNS approach.

Full details of a comprehensive global analysis using this procedure will be presented in a future publication. However, here we will concentrate on those data which describe the charm contribution to the structure function only. The component $F_{2, c}$ has been measured at intermediate $x$ values by European Muon Collaboration (EMC) [32] (via the detection of inclusive muons) and at small $x$ by the H1 [4] and ZEUS [5] collaborations at HERA (through measuring $D$ and $D^{*}$ cross sections). The latter indicate that as much as 20-25\% of the total $F_{2}\left(x, Q^{2}\right)$ is due to $F_{2, c}\left(x, Q^{2}\right)$. While these data on $F_{2, c}\left(x, Q^{2}\right)$ are not included as part of the general fitting procedure, we can compare them with our theoretical predictions. The results are shown in Fig. 5. A very good description of both the small and medium $x$ data is achieved for a charm mass of $m_{c}=1.35 \mathrm{GeV},{ }^{10}$ although there is a strong suggestion that a slightly higher mass would be preferred, i.e., the curves for $m_{c}=1.5 \mathrm{GeV}$ give a rather better description. In fact it is the data for $Q^{2} \sim 2 \mathrm{GeV}^{2}$ which strongly favor this higher value of $m_{c}$. Since in this region of $Q^{2}$ the theoretical approach is unambiguous, i.e., the true result must be very similar to the FFNS calculation, these points may be thought of as determining the value of

\footnotetext{
${ }^{9}$ The fit is not as good as the leading order renormalization scale consistent (LORSC) fit $[31,20]$, which includes $\ln (1 / x)$ corrections, but not yet charm mass corrections.

${ }^{10}$ There is also a single EMC data point at $x=0.422$ and $Q^{2}=78 \mathrm{GeV}^{2}$ not shown in Fig. 5, which has $F_{2, c}\left(x, Q^{2}\right)$ $=0.00274 \pm 0.00152$ compared to a prediction of 0.0003 .
}

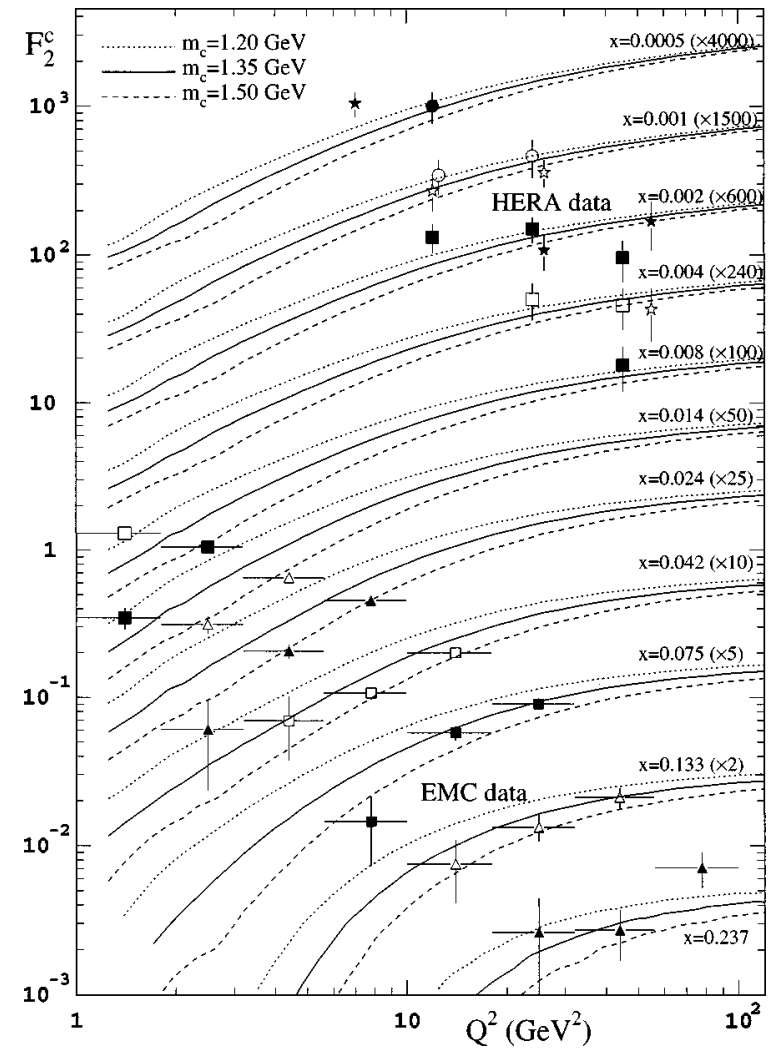

FIG. 5. Our prediction for $F_{2, c}\left(x, Q^{2}\right)$ using our NLO prescription, the NLO partons obtained from our global fit, and three different values from $m_{c}$ compared with the EMC and HERA data.

$m_{c}$ at about $1.5 \mathrm{GeV}$, and a value very similar to this that should be used over the whole range.

One can compare our results with those for the FFNS and ZM-VFNS shown in Fig. 5 of [26] (with $m_{c}=1.5 \mathrm{GeV}$ ). From this figure it seems as though the FFNS tends to undershoot most data points, particularly the EMC points at moderate $x$, though this could be improved by choosing lower $m_{c}$. The ZM-VFNS curve matches HERA data well, but is clearly the wrong shape for the EMC data, especially those points at $Q^{2}<3 \mathrm{GeV}^{2}$ not shown in this figure, where the ZM-VFNS fails hopelessly. This is only what one would expect from the construction of the ZM-VFNS, i.e., a disregard for the correct physics near threshold. Hence, the EMC charm data in particular seem to favor a VFNS calculation. The HERA data on charm itself are not yet precise enough to distinguish strongly between approaches, but as seen above, the fact that the charm component is a large part of the total $F_{2}\left(x, Q^{2}\right)$ values means that global fits are more discriminating, and favor the VFNS approach. Illustrating this final point, in Fig. 6 we show the relative importance of the charm and bottom components to the total structure function, and note the large fraction which is due to charm in the HERA low $x$ region. The bottom contributes no more than $\sim 4 \%$ in any currently accessible range of $x$ and $Q^{2}$.

\section{THE LONGITUDINAL STRUCTURE FUNCTION}

Finally we discuss our prediction for the heavy quark contribution to the longitudinal structure function. Although there are no data directly available on this quantity, we feel that it is an important issue. This is because the implemen- 

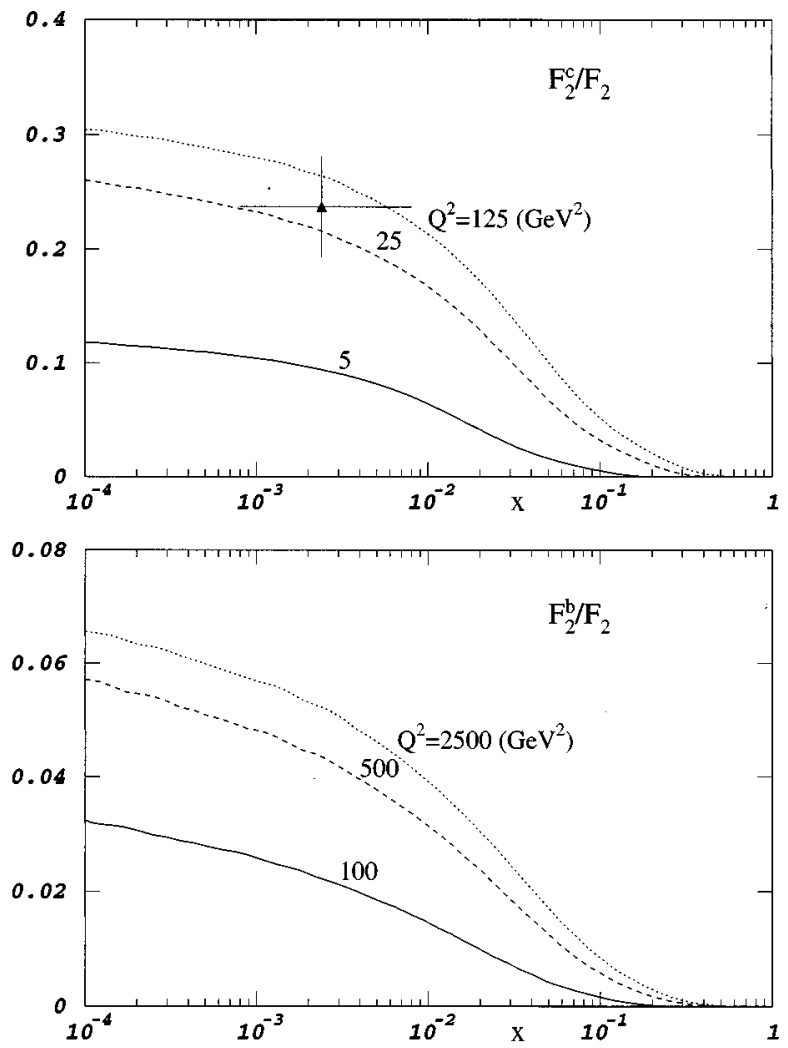

FIG. 6. The ratios $F_{2, c} / F_{2}$ and $F_{2, b} / F_{2}$ at fixed values of $Q^{2}$ resulting from our NLO parton distributions and taking $m_{c}$ $=1.35 \mathrm{GeV}$ and $m_{b}=4.3 \mathrm{GeV}$. The experimental data point shows the estimate from Ref. [4] for $F_{2, c} / F_{2}$ in the kinematic range $10 \mathrm{GeV}^{2}<Q^{2}<100 \mathrm{GeV}^{2}$.

tation is a little different from the case of $F_{2}\left(x, Q^{2}\right)$, also because the charm contribution has a very important bearing on the total longitudinal structure function, contributing up to about 35\% in the ZM-VFNS for example, and finally because our results are very different from those in other ways of implementing a variable flavor number scheme.

As for $F_{2}\left(x, Q^{2}\right)$, and for the same reasons, there is no way we can obtain the (hypothetical) absolutely correctly ordered expression. Therefore, as in this previous case we want some relatively simple prescription which will reflect the physics involved correctly. There is a lot of similarity between our order-by-order prescription for the heavy quark contribution to $F_{L}\left(x, Q^{2}\right)$ and that for $F_{2}\left(x, Q^{2}\right)$, and the equations that the VFNS coefficient functions must satisfy in terms of the operator matrix elements and the FFNS coefficient functions are once again Eqs. (3.6)-(3.10). One large difference between the two is the fact that in a zero-mass formulation there are no zeroth-order in $\alpha_{s}\left(Q^{2}\right)$ coefficient functions for the longitudinal structure function, and hence the $\mathcal{O}\left(\alpha_{s}\left(Q^{2}\right)\right)$ coefficient functions are leading order and renormalization scheme independent. All previous implementations of a VFNS [22,23,28] have included a zerothorder heavy quark longitudinal coefficient function, i.e., the term in Eq. (4.24) which $\propto 4 M^{2} / Q^{2}$. This procedure means that there is a coefficient function at lower order than the one which becomes leading order in the ZM-VFNS limit, and hence in order to reach this limit with the LO VFNS expression coefficient functions at both zeroth and first order in $\alpha_{s, n_{f+1}}\left(Q^{2}\right)$ would need to be included. Also, if one includes any zeroth-order coefficient function, using the expression (3.6) for the $\mathcal{O}\left(\alpha_{s, n_{f+1}}\left(Q^{2}\right)\right)$ gluon coefficient function results in $C_{L, H g}^{\mathrm{VF}, 1}\left(z, Q^{2} / M^{2}\right)$ having a component which is renormalization scheme dependent.

Hence, we choose not to have any zeroth-order contribution to the longitudinal coefficient functions. As with $F_{2}\left(x, Q^{2}\right)$ our VFNS coefficient functions are then determined entirely by the requirements of reduction to the ZMVFNS order by order as $Q^{2} \rightarrow \infty$ and continuity with the FFNS across the boundary $Q^{2}=M^{2}$. Therefore, the prescription for the VFNS longitudinal structure function is very similar to that for $F_{2}\left(x, Q^{2}\right)$, except that the relative order of heavy quark coefficient functions above $M^{2}=Q^{2}$ and light quark coefficients at all $Q^{2}$ is one higher, i.e., $\mathcal{O}\left(\alpha_{s, n_{f+1}}\left(Q^{2}\right)\right)$ is leading order, etc. The prescription for the FFNS structure function at fixed order is then very straightforward, i.e.,

$$
\begin{aligned}
F_{L, i}^{n}\left(x, Q^{2}\right)= & \sum_{m=0}^{n} \sum_{a}\left(\frac{\alpha_{s, n_{f}}\left(Q^{2}\right)}{2 \pi}\right)^{n-m+1} \\
& \times C_{L, i a}^{\mathrm{FF}(n-m+1)}\left(M^{2} / Q^{2}\right) \otimes f_{m, a}^{n_{f}}\left(Q^{2}\right), \\
& n=0 \rightarrow \infty,
\end{aligned}
$$

for both the heavy and light quark structure functions. The general form of the expression above $Q^{2}=M^{2}$ is the same as this, i.e.,

$$
\begin{aligned}
F_{L, i}^{n}\left(x, Q^{2}\right)= & \sum_{m=0}^{n} \sum_{a}\left(\frac{\alpha_{s, n_{f}+1}\left(Q^{2}\right)}{2 \pi}\right)^{n-m+1} \\
& \times C_{L, i b}^{\mathrm{VF}, n-m+1}\left(M^{2} / Q^{2}\right) \otimes f_{m, b}^{n_{f}+1}\left(Q^{2}\right), \\
& n=0 \rightarrow \infty .
\end{aligned}
$$

Since the expressions are now of an identical form both sides of the transition point [which was impossible for $F_{2}\left(x, Q^{2}\right)$ because of the requirement of zeroth-order heavy quark coefficient functions above $Q^{2}=M^{2}$ ], and are identical to all orders, continuity of the structure functions themselves is guaranteed order by order in $\alpha_{s}\left(Q^{2}\right)$ across the transition point. However, as in the case of $F_{2}\left(x, Q^{2}\right)$ the heavy quark coefficient functions at each order have to be determined using some prescription. (This ambiguity has no effect on the continuity of the structure function since at $n$th order in the expression for the structure function the $n$ th-order heavy quark coefficient functions only appear coupling to the zeroth-order heavy quark distribution, which vanishes at $Q^{2}=M^{2}$.)

As with $F_{2}\left(x, Q^{2}\right)$ it would be nice to demand both continuity of the structure function and its $\ln Q^{2}$ derivative across the transition point. Since the expressions for the structure function are of exactly the same form both above and below $Q^{2}=M^{2}$ in this case (essentially because there are no zeroth-order terms in the longitudinal structure function), we can now attempt to equate the $\ln Q^{2}$ derivatives of the $n$ th-order terms in both expressions rather than making the derivative of the $n$ th-order VFNS expression match on to the $n$ th-order derivative in the FFNS as was necessary for $F_{2}\left(x, Q^{2}\right)$. As in the previous case we have enough choice to 
demand only that this is true in the gluon sector, but again by far the dominant contribution to this derivative comes from this sector. However, now we have an additional problem compared to the previous case. This can be seen by examining the lowest-order expressions.

In the FFNS the lowest-order expression for the heavy quark structure function is

$$
F_{L, H}^{0}\left(x, Q^{2}\right)=\frac{\alpha_{s, n_{f}}\left(Q^{2}\right)}{2 \pi} C_{L, H g}^{\mathrm{FF} 1}\left(M^{2} / Q^{2}\right) \otimes g_{0}^{n_{f}}\left(Q^{2}\right),
$$

while in the VFNS, it is

$$
\begin{aligned}
F_{L, H}^{0}\left(x, Q^{2}\right)= & \frac{\alpha_{s, n_{f}+1}\left(Q^{2}\right)}{2 \pi} C_{L, H g}^{\mathrm{VF}, 1}\left(M^{2} / Q^{2}\right) \otimes g_{0}^{n_{f}+1}\left(Q^{2}\right) \\
& +C_{L, H H}^{\mathrm{VF}, 1}\left(M^{2} / Q^{2}\right) \otimes\left(H\left(Q^{2}\right)+\bar{H}\left(Q^{2}\right)\right)_{0}^{n_{f}+1},
\end{aligned}
$$

and from Eq. (3.6) we have

$$
\begin{aligned}
C_{L, H g}^{\mathrm{VF}, 1}\left(z, M^{2} / Q^{2}\right) & =C_{L, H g}^{\mathrm{FF}, 1}\left(z, M^{2} / Q^{2}\right) \\
& =\left[4 z(1-z) v-8 \epsilon z^{2} \ln \left(\frac{1+v}{1-v}\right)\right],
\end{aligned}
$$

for the charm quark, where again $\epsilon=m_{c}^{2} / Q^{2}$ and $\theta\left(\hat{W}^{2}\right.$ $-4 m_{c}^{2}$ ) is implied whenever $v$ appears. Clearly the structure functions are the same at $Q^{2}=M^{2}$. It is also clear that the $\mathcal{O}\left(\alpha_{s}\left(Q^{2}\right)\right)$ expression for the $\ln Q^{2}$ derivative is the same on both sides, i.e.,

$$
\frac{d F_{L, H}\left(x, Q^{2}\right)}{d \ln Q^{2}}=\frac{\alpha_{s}\left(Q^{2}\right)}{2 \pi} \frac{d C_{L, H g}^{\mathrm{FF}, 1}\left(Q^{2} / M^{2}\right)}{d \ln Q^{2}} \otimes g_{0}\left(Q^{2}\right) .
$$

However, this expression, which vanishes as $Q^{2} / M^{2} \rightarrow \infty$, is lower order than the leading-order asymptotic expansion, which is $\mathcal{O}\left(\alpha_{s}^{2}\left(Q^{2}\right)\right)$. It is this, rather than a zeroth-order coefficient function, which truly reflects the fact that the heavy quark longitudinal structure function has behavior, which begins at lower order than the massless expression. This $\mathcal{O}\left(\alpha_{s}\left(Q^{2}\right)\right)$ derivative means that while the asymptotic $\mathcal{O}\left(\alpha_{s}^{2}\left(Q^{2}\right)\right)$ expression for the total derivative of the longitudinal structure function is renormalization scheme independent, it contains a part which vanishes as $Q^{2} / M^{2} \rightarrow \infty$ which is renormalization scheme dependent. This is different to the case for $F_{2, H}\left(x, Q^{2}\right)$, where the leading asymptotic and $\mathcal{O}\left(M^{2} / Q^{2}\right)$ parts of the derivative are both of the same order, i.e., $\mathcal{O}\left(\alpha_{s}\left(Q^{2}\right)\right)$.

If we treat the $\mathcal{O}\left(\alpha_{s}\left(Q^{2}\right)\right)$ component of $d F_{L, H}\left(x, Q^{2}\right) / d \ln Q^{2}$ as a superleading part which is trivially continuous across $Q^{2}=M^{2}$, and then examine the form of the $\mathcal{O}\left(\alpha_{s}^{2}\left(Q^{2}\right)\right)$ terms coming from the derivatives of Eqs. (7.3) and (7.4), then since each of the leading-order expressions is renormalization scheme independent, then so are the contributions obtained in the expressions for the derivatives. Explicitly we obtain, in the FFNS,

$$
\begin{aligned}
\frac{d F_{L, H}\left(x, Q^{2}\right)}{d \ln Q^{2}}= & \left(\frac{\alpha_{s, n_{f}}\left(Q^{2}\right)}{2 \pi}\right)^{2} C_{L, H g}^{\mathrm{FF}, 1}\left(Q^{2} / M^{2}\right) \\
& \otimes\left(-\beta_{0}^{n_{f}} g_{0}^{n_{f}}\left(Q^{2}\right)+P_{g g}^{0, n_{f}} \otimes g_{0}^{n_{f}}\left(Q^{2}\right)\right. \\
& \left.+P_{g q}^{0} \otimes \Sigma_{0}^{n_{f}}\left(Q^{2}\right)\right),
\end{aligned}
$$

and in the VFNS

$$
\begin{aligned}
\frac{d F_{L, H}\left(x, Q^{2}\right)}{d \ln Q^{2}}= & \left(\frac{\alpha_{s, n_{f}+1}\left(Q^{2}\right)}{2 \pi}\right)^{2}\left\{C_{L, H g}^{\mathrm{VF}, 1}\left(Q^{2} / M^{2}\right)\right. \\
& \otimes\left(-\beta_{0}^{n_{f}+1} g_{0}^{n_{f}}\left(Q^{2}\right)+P_{g g}^{0, n_{f}+1} \otimes g_{0}^{n_{f}}\left(Q^{2}\right)\right. \\
& \left.+P_{g q}^{0} \otimes \Sigma_{0}^{n_{f}}\left(Q^{2}\right)\right)+C_{L, H H}^{\mathrm{VF}, 1}\left(Q^{2} / M^{2}\right) \\
& \otimes\left[P_{q g}^{0} \otimes g_{0}^{n_{f}+1}\left(Q^{2}\right)\right. \\
& \left.+P_{q q}^{0} \otimes\left(H\left(Q^{2}\right)+\bar{H}\left(Q^{2}\right)\right)_{0}\right] \\
& \left.-\beta_{0}^{n_{f}+1}\left(H\left(Q^{2}\right)+\bar{H}\left(Q^{2}\right)\right)_{0}\right\}
\end{aligned}
$$

From previous arguments it is clear that the terms $\propto C_{L, H g}^{\mathrm{VF}, 1}\left(z, Q^{2} / M^{2}\right)$ in each equation are equal at $Q^{2}=M^{2}$. The vanishing of the heavy quark distribution at this scale, leads to the single condition

$$
C_{L, H H}^{\mathrm{VF}, 1}(z, 1)=0,
$$

in order to match these $\mathcal{O}\left(\alpha_{s}^{2}\left(Q^{2}\right)\right)$ contributions to the derivative. Thus, we have this condition, along with the fact that $C_{L, H H}^{\mathrm{VF}, 1}\left(z, Q^{2} / M^{2}\right)$ must reduce to the correct asymptotic form, in order to determine $C_{L, H H}^{\mathrm{VF}, 1}\left(z, Q^{2} / M^{2}\right)$. It is clearly possible to choose forms for $C_{L, H H}^{\mathrm{VF}, 1}\left(z, Q^{2} / M^{2}\right)$ which satisfy these conditions, but there is rather less guidance as to the precise form required than for $F_{2, H}\left(x, Q^{2}\right)$, where the condition at $Q^{2}=M^{2}$ contained a component, which was clearly identifiable as the asymptotic expression.

This indeterminacy is due to the fact that the $\mathcal{O}\left(\mathrm{M}^{2} / Q^{2}\right)$ contributions to the derivative begin at one lower order than the asymptotic form, rather than our chosen manner of imposing the matching. If we had chosen to match Eq. (7.8) to the total $\mathcal{O}\left(\alpha_{s}^{2}\left(Q^{2}\right)\right)$ expression for $d F_{L, H}\left(x, Q^{2}\right) / d \ln Q^{2}$ in the FFNS, rather than just the part coming from Eq. (7.7), i.e., analogously to $F_{2, H}\left(x, Q^{2}\right)$, we would have encountered a different problem. In this case the determined value of $C_{L, H H}^{\mathrm{VF}, 1}(z, 1)$ would have contained a part $\propto d C_{L, H g}^{\mathrm{FF}, 2}\left(z, Q^{2} / M^{2}\right) / d \ln Q^{2}$, which contains $C_{L, q q}^{\mathrm{VF}, n_{f}+1,1}(z)$, and the asymptotic limit would therefore appear more naturally. However, the full expression for $d C_{L, H q}^{\mathrm{FF}, 2}\left(z, Q^{2} / M^{2}\right) / d \ln Q^{2}$, and consequently the full expression for $C_{L, H H}^{\mathrm{VF}, 1}\left(z, Q^{2} / M^{2}\right)$ implied, contains $\mathcal{O}\left(M^{2} / Q^{2}\right)$ parts which are renormalization scheme dependent [since they are subleading to the $\mathcal{O}\left(\alpha_{s}\left(Q^{2}\right)\right)$ expression]. This is not satisfactory in the definition of the leading order VFNS coefficient function, and the renormalization scheme dependent part of the expression should be removed. However, there is no unique way to do this, and hence the definition of $C_{L, H H}^{\mathrm{VF}, 1}\left(z, M^{2} / Q^{2}\right)$ would be just as ambiguous as when using our chosen matching condition. 
Hence, we have to live with the fact that there is no completely satisfactory way to determine $C_{L, H H}^{\mathrm{VF}, 1}\left(z, Q^{2} / M^{2}\right)$ from physical arguments. We choose to impose Eq. (7.9), as well as the fact that $C_{L, H H}^{\mathrm{VF}, 1}\left(z, Q^{2} / M^{2}\right)$ must reduce to the correct asymptotic form, and also choose the coefficient function so that a smooth threshold in $\hat{W}^{2}$ is automatically incorporated. A simple choice satisfying all these requirements is

$$
C_{L, H H}^{\mathrm{VF}, 1}\left(z, Q^{2} / M^{2}\right)=\frac{8}{3} v\left(1-M^{2} / Q^{2}\right) z .
$$

In practice this ambiguity has little effect phenomenologically, since the vast majority of the LO expression for $F_{L, H}^{0}\left(x, Q^{2}\right)$ comes from the gluon contribution which is determined uniquely. Using Eq. (3.6) we have now also defined $C_{L, H g}^{\mathrm{VF}, 2}\left(z, Q^{2} / M^{2}\right)$, i.e.,

$$
\begin{aligned}
C_{L, H g}^{\mathrm{VF}, 2}\left(z, Q^{2} / M^{2}\right)= & C_{L, H g}^{\mathrm{FF}, 2}\left(z, Q^{2} / M^{2}\right)-\ln \left(Q^{2} / M^{2}\right) P_{q g}^{0} \\
& \otimes C_{L, H H}^{\mathrm{VF}, 1}\left(Q^{2} / M^{2}\right),
\end{aligned}
$$

in $\overline{\mathrm{MS}}$ scheme, although we do not have to make use of this yet. The fact that $C_{L, H H}^{\mathrm{VF}, 1}\left(z, Q^{2} / M^{2}\right)$ reduces to the correct asymptotic limit guarantees that $C_{L, H g}^{\mathrm{VF}, 2}\left(z, Q^{2} / M^{2}\right)$ does.

As far as the light quark contribution is concerned, the coefficient functions are identical above and below $Q^{2}$ $=M^{2}$, and the only effect is the change of the evolution of the parton distributions and in the running of the coupling. The $\ln Q^{2}$ derivatives of these LO light quark distributions which are entirely of $\mathcal{O}\left(\alpha_{s}^{2}\left(Q^{2}\right)\right)$, are not quite continuous across the transition point because of the flavor dependence of $\beta_{0}$ and of the lowest-order splitting functions, i.e., of $P_{g g}^{0}(z)$. As in the $\mathcal{O}\left(\alpha_{s}^{2}\left(Q^{2}\right)\right)$ derivative for $F_{2, i}\left(x, Q^{2}\right)$, there is continuity in the gluon sector, but not in the quark sector. Phenomenologically the discontinuity is very small, and becomes formally smaller as we work to higher orders.

The result of our leading-order calculation of $F_{L, c}\left(x, Q^{2}\right)$, using the same $\mathrm{LO}$ parton distributions as before, is shown in Fig. 7, along with the LO FFNS and the LO ZM-VFNS results. As in the case of $F_{2, c}\left(x, Q^{2}\right)$ one can see that the transition from the FFNS result is extremely smooth, and of course, the the correct asymptotic limit is reached. We note that at low $Q^{2}$ the VFNS result for $F_{L, c}\left(x, Q^{2}\right)$ is very different indeed from that in the ZM-VFNS. This leads to a very significant difference between the results for the total $F_{L}\left(x, Q^{2}\right)$ in the two different schemes, and important phenomenological implications. We also show explicitly the contribution made by the charm quark distribution. At high $Q^{2}$ this is unambiguously defined, and at low $Q^{2}$ it is very small indeed. Therefore, the ambiguity in the low $Q^{2}$ heavy quark contribution is not really significant.

A very important difference should be noted between this approach and previous VFNS approaches at this point. As already mentioned, all previous approaches have used a zeroth-order charm quark coefficient function of the form

$$
\hat{C}_{L, c c}^{\mathrm{VF}, 0}(z, \epsilon)=4 \operatorname{gez} \delta\left(\hat{x}_{0}-z\right) .
$$

If one were to regard the $\mathrm{LO}$ expression for $F_{L, c}\left(x, Q^{2}\right)$ as just this coefficient function convoluted with the heavy quark distribution function then the behavior would be rather strange, having a sharp threshold at $Q^{2}=m_{c}^{2}$, growing
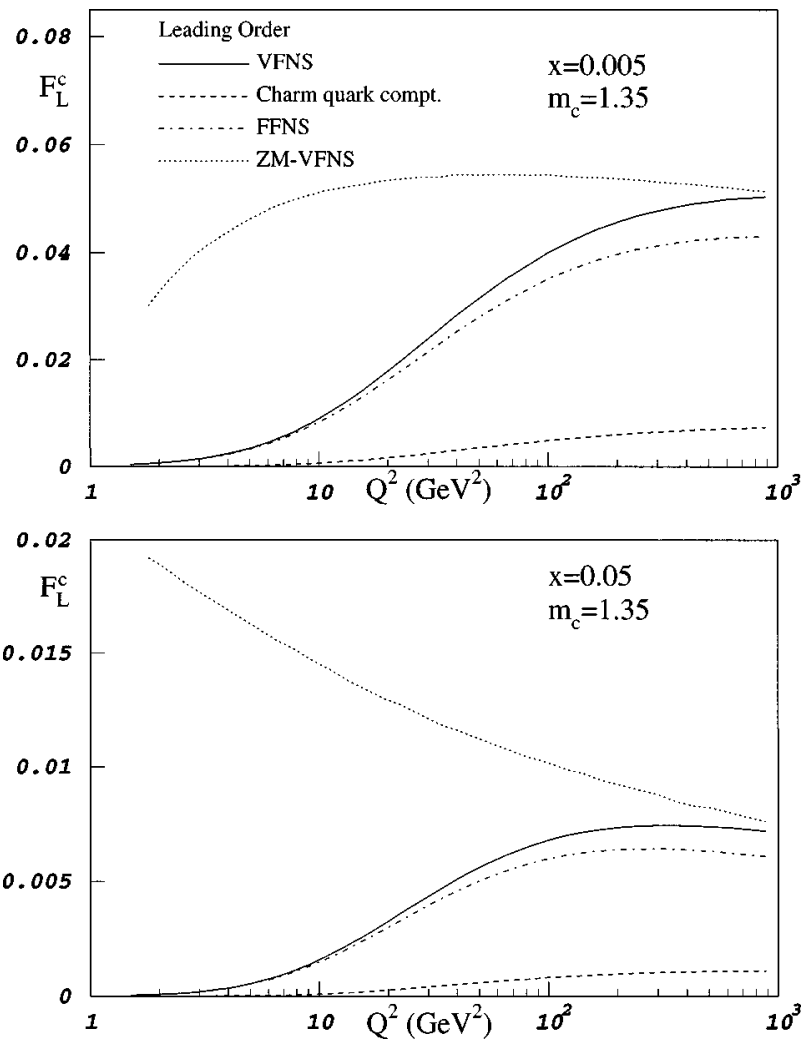

FIG. 7. Charm quark structure function, $F_{L, c}\left(x, Q^{2}\right)$ for $x$ $=0.05$ and $x=0.005$ calculated using our LO prescription, our input parton distributions evolved at LO and renormalization scale $\mu^{2}=Q^{2}$. Also shown are the continuation of the LO FFNS expression and the ZM-VFNS expression both calculated using the same parton distributions and same choice of scale.

quickly, then turning over and going to zero as $Q^{2} / m_{c}^{2}$ $\rightarrow \infty$. If, as is more likely, the LO expression is taken to include both the zeroth-order and $\mathcal{O}\left(\alpha_{s}\left(Q^{2}\right)\right)$ coefficient functions, so that the correct asymptotic LO limit is reached, then $\hat{C}_{L, c g}^{\mathrm{VF}, 1}(z, \epsilon)$ is defined by Eq. (3.12), i.e.,

$$
\begin{aligned}
\hat{C}_{L, c g}^{\mathrm{VF}, 1}(z, \epsilon)= & \hat{C}_{L, c g}^{\mathrm{F}, 1}(z, \epsilon)-\frac{4 m_{c}^{2}}{Q^{2}} P_{q g}^{0} \otimes z \delta\left(\hat{x}_{0}-z\right) \\
& \times\left(\ln \left(Q^{2} / m_{c}^{2}\right)+c_{\mathrm{rs}}\right) .
\end{aligned}
$$

As well as this introducing incorrect renormalization scheme dependence into a leading-order expression (via $c_{\mathrm{rs}}$ ), it has unfortunate phenomenological consequences. The VFNS differs from the FFNS expression by

$$
\begin{aligned}
4 \epsilon z \delta\left(\hat{x}_{0}-z\right) \otimes[ & \left(c\left(Q^{2}\right)+\bar{c}\left(Q^{2}\right)\right)_{0}-\alpha_{s, 4}\left(Q^{2}\right) P_{q g}^{0} \\
& \left.\times \ln \left(Q^{2} / m_{c}^{2}\right) \otimes g_{0}^{4}\left(Q^{2}\right)\right],
\end{aligned}
$$

where we have used $\overline{\mathrm{MS}}$ scheme. These two terms are intended to largely cancel at and just above $Q^{2}=m_{c}^{2}$, ensuring a relatively smooth transition as in the ACOT prescription for the $\mathrm{LO}$ expression for $F_{2, c}\left(x, Q^{2}\right)$. The procedure works well in the case of $F_{2, c}\left(x, Q^{2}\right)$, and the transition is quite smooth, as we have seen. However, the cancellation is not exact (otherwise we would just have the FFNS), $\left(c\left(z, Q^{2}\right)\right.$ $\left.+\bar{c}\left(z, Q^{2}\right)\right)_{0} \approx \alpha_{s, 4}\left(Q^{2}\right) P_{q g}^{0} \ln \left(Q^{2} / m_{c}^{2}\right) \otimes g_{0}^{4}\left(Q^{2}\right)$ for $Q^{2}$ just 
above $m_{c}^{2}$, but the resummation of the logs in the evolution of the charm quark distribution leads to differences appearing. To a rough approximation,

$$
\begin{aligned}
& \left(c\left(z, Q^{2}\right)+\bar{c}\left(z, Q^{2}\right)\right)_{0}-\alpha_{s, 4}\left(Q^{2}\right) P_{q g}^{0} \ln \left(Q^{2} / m_{c}^{2}\right) \otimes g_{0}^{4}\left(Q^{2}\right) \\
& \quad \approx\left(\alpha_{s, 4}\left(Q^{2}\right) \ln \left(Q^{2} / m_{c}^{2}\right)\right)^{2} P_{q g}^{0} \otimes P_{g g}^{0,4} \otimes g_{0}^{4}\left(Q^{2}\right)
\end{aligned}
$$

at moderate $Q^{2}$. Inserting this into Eq. (7.14) leads to

$$
4 \epsilon\left(\alpha_{s, 4}\left(Q^{2}\right) \ln \left(Q^{2} / m_{c}^{2}\right)\right)^{2} z \delta\left(\hat{x}_{0}-z\right) \otimes P_{q g}^{0} \otimes P_{g g}^{0,4} \otimes g_{0}^{4}\left(Q^{2}\right) .
$$

For $Q^{2} \approx 5-10 \mathrm{GeV}^{2}$ this expression is comparable in size to the FFNS component of the full expression for $F_{L, c}\left(x, Q^{2}\right)$, which $\sim \alpha_{s} g_{0}^{4}\left(Q^{2}\right)$ with damping due to kinematic factors [and which is more than 10 times smaller than the LO FFNS component for $\left.F_{2, c}\left(x, Q^{2}\right)\right]$. However, it falls away quickly at larger $Q^{2}$. This leads to the LO VFNS expression for $F_{L, c}\left(x, Q^{2}\right)$ increasing very quickly above the FFNS expression above the transition point, dramatically slowing, or perhaps even falling at $Q^{2} \sim 5 m_{c}^{2}$, and then smoothly approaching the correct asymptotic limit. I.e., there is a very pronounced unphysical bulge in the value of $F_{L, c}\left(x, Q^{2}\right)$ calculated in this way. When one calculates $R_{c}=F_{L, c} /\left(F_{2, c}\right.$ $-F_{L, c}$ ), which exhibits the relative rate of growth of $F_{L, c}$ and $F_{2, c}$, the effect is demonstrated much more clearly as a distinct hump peaking at about $Q^{2}=3 m_{c}^{2}$. This can be seen very clearly in Fig. 9 of [28], where the effect is particularly dramatic, since the evolution of the heavy quark distribution there is even quicker than in $\overline{\mathrm{MS}}$, and is at NLO. However, the treatment of coefficient functions follows the same general principles as ACOT, and the same type of effect, if somewhat smaller (the reduction depending very strongly on the particular choice of renormalization scale-one which departs extremely slowly from $m_{c}^{2}$ as $Q^{2}$ increases could remove the effect) will be clearly seen in their expressions. ${ }^{11}$ Even in the absence of detailed data, this type of effect seems sufficient to rule out this approach as a suitable way to order a VFNS expression.

We now consider the NLO expressions for the longitudinal structure functions. For both heavy and light quark structure functions both above and below $Q^{2}=M^{2}$, we add to the LO expressions the $\mathcal{O}\left(\alpha_{s}^{2}\left(Q^{2}\right)\right)$ coefficient functions convoluted with the LO parton distributions and the $\mathcal{O}\left(\alpha_{s}\left(Q^{2}\right)\right)$ coefficient functions convoluted with the NLO parton distributions. Let us first consider the heavy quark coefficient function. It is guaranteed by satisfying Eqs. (3.6)-(3.10) order by order in $\alpha_{s}\left(Q^{2}\right)$, while also satisfying the correct relations between parton distributions and the coupling, that this procedure will lead to structure functions, which are continuous across $Q^{2}=M^{2}$. This is straightforward, if a little tedious to check. Continuity of the derivative of the heavy quark structure function across the threshold is not guaranteed, but depends on the particular choice of the heavy quark coefficient functions. We can compare the derivatives of the

\footnotetext{
${ }^{11}$ In fact, since as we see in Fig. 1 at $x=0.005$ the subtraction term is larger than the heavy parton distribution, the effect will be negative.
}

full NLO expressions in both the FFNS and VFNS up to $\mathcal{O}\left(\alpha_{s}^{3}\left(Q^{2}\right)\right)$. From the conditions we have already imposed using Eqs. (3.6)-(3.10), it is guaranteed that all new terms we introduce which behave like $\alpha_{s}^{2}\left(Q^{2}\right)$, i.e., those depending on $d C_{L, H g(q)}^{\mathrm{F}(\mathrm{V}) \mathrm{R}, 2} / d \ln Q^{2}$, will be continuous across the transition point. Again this is straightforward to check. If we examine the $\mathcal{O}\left(\alpha_{s}^{3}\left(Q^{2}\right)\right)$ contributions to the expressions then both in the FFNS and VFNS these are very involved, i.e., containing rather more terms than Eqs. (4.10) and (4.11). However, as with Eqs. (4.10) and (4.11) many of these terms vanish at $Q^{2}=M^{2}$, because the heavy parton distribution vanishes here, also because in this case $C_{L, H H}^{\mathrm{VF}, \mathrm{NS}, 1}(z, 1)=0$, and also because many other terms are the same in both expressions. A long, but entirely straightforward calculation reveals that if we require continuity of the derivative in the gluon sector, we have the requirement that

$$
\begin{aligned}
C_{L, H H}^{\mathrm{VF}, \mathrm{S}, 2}\left(Q^{2} / M^{2}\right) \otimes P_{q g}^{0}= & C_{L, H g}^{\mathrm{FF}, 2}\left(z, Q^{2} / M^{2}\right)\left(\beta_{0}^{n_{f}+1}-\beta_{0}^{n_{f}}\right) \\
& +C_{L, H g}^{\mathrm{FF}, 1}\left(z, Q^{2} / M^{2}\right)\left(\beta_{1}^{n_{f}+1}-\beta_{1}^{n_{f}}\right) \\
& -C_{L, H g}^{\mathrm{FF}, 1}\left(Q^{2} / M^{2}\right) \\
& \otimes\left(P_{g g}^{1, n_{f}+1}-P_{g g}^{1, n_{f}}\right)
\end{aligned}
$$

at $Q^{2}=M^{2}$. So at $\mathcal{O}\left(\alpha_{s}^{2}\left(Q^{2}\right)\right)$, as at $\mathcal{O}\left(\alpha_{s}\left(Q^{2}\right)\right)$, there is no implication of the asymptotic form required of the heavy quark coefficient function in the condition at $Q^{2}=M^{2}$, but the condition is no longer that the coefficient function is zero at this value of $Q^{2}$. We can understand where the nonzero terms come from quite easily. If we had used the whole of the $\mathcal{O}\left(\alpha_{s, n_{f}}^{3}\left(Q^{2}\right)\right)$ expression for the derivative of the heavy quark structure function in the FFNS, and equated this to our VFNS expression, then the asymptotic form of $C_{L, H H}^{\mathrm{FF}, 2}\left(z, Q^{2} / M^{2}\right)$ would have appeared naturally in the expression for $d C_{L, H g}^{\mathrm{FF}, 3} / d \ln Q^{2}$. However, by examination of expression for $d C_{L, H g}^{\mathrm{FF}, 3} / d \ln Q^{2}$ contained within Eq. (3.6), we would find that the definition of $C_{L, H H}^{\mathrm{VF}, \mathrm{NS}, 2}\left(z, Q^{2} / M^{2}\right)$ would also need to contain terms of the sort in Eq. (7.17), as well as others which vanish at $Q^{2}=M^{2}$, in order to reduce to the correct asymptotic limit. However, in an analogous fashion to our previous discussion at leading order, we do not use this technique since parts of the $\mathcal{O}\left(M^{2} / Q^{2}\right)$ corrections to $d C_{L, H g}^{\mathrm{FF}, 3} / d \ln Q^{2}$ are properly of NNLO, i.e., are renormalization scheme dependent in such a way as to compensate for the renormalization scheme variation of the NLO terms. This would require an ambiguous subtraction procedure for these terms, and we would have no more real information than that contained in Eq. (7.17) and the asymptotic condition.

Hence, as for the $\mathcal{O}\left(\alpha_{s, n_{f}+1}\left(Q^{2}\right)\right)$ coefficient function, we make a simple choice for the coefficient function which satisfies Eq. (7.17), which reduces to the correct asymptotic limit, and which explicitly contains the correct threshold behavior. Once again we multiply the asymptotic limit, which makes no appearance at $Q^{2}=M^{2}$, by $\left(1-M^{2} / Q^{2}\right) v$. We multiply the terms appearing in Eq. (7.17), but which must disappear asymptotically, by $M^{2} / Q^{2}$ (in this case the threshold behavior is automatically contained in the expressions). Hence we obtain 


$$
\begin{aligned}
C_{L, H H}^{\mathrm{VF}, \mathrm{S}, 2}\left(z, Q^{2} / M^{2}\right)= & \left(1-\frac{M^{2}}{Q^{2}}\right) v C_{L, q q}^{n_{f}^{+1,2}}(z)+\frac{M^{2}}{Q^{2}}\left(P_{q g}^{0}\right)^{-1} \\
& \otimes\left[C_{L, H g}^{\mathrm{FF}, 2}\left(Q^{2} / M^{2}\right)\left(\beta_{0}^{n_{f}+1}-\beta_{0}^{n_{f}}\right)\right. \\
& +C_{L, H g}^{\mathrm{FF}, 1}\left(Q^{2} / M^{2}\right)\left(\beta_{1}^{n_{f}+1}-\beta_{1}^{n_{f}}\right) \\
& \left.-C_{L, H g}^{\mathrm{FF}, 1}\left(Q^{2} / M^{2}\right) \otimes\left(P_{g g}^{1, n_{f}+1}-P_{g g}^{1, n_{f}}\right)\right] .
\end{aligned}
$$

This definition is ambiguous at low $Q^{2}$, but as at leading order the total heavy quark structure function at NLO is totally dominated by the gluon contribution. We also note that the ambiguity introduced at LO from the definition of the heavy quark coefficient function is very largely negated at NLO by the inclusion of $C_{L, H H}^{\mathrm{VF}, \mathrm{NS}, 1}\left(z, Q^{2} / M^{2}\right)$ in the expression for $C_{H g}^{\mathrm{VF}, 2}\left(z, Q^{2} / M^{2}\right)(7.11)$. As we work to higher orders, the ambiguity formally disappears. We also note that the coefficient function $C_{L, H H}^{\mathrm{VF}, \mathrm{S}, 2}\left(z, Q^{2} / M^{2}\right)$ is the sum of the nonsinglet and pure singlet coefficient functions. We are free to separate them as we wish, using the condition that each tends to the correct asymptotic limit. It would also be desirable to choose each so that they respect the kinematic threshold. The choice has no bearing on the expression for the structure function, but a simple choice is to let the nonsinglet part contain all parts $\propto v$, and to split the other part simply in terms of the asymptotic form.

Comparing the $\mathcal{O}\left(\alpha_{s}^{3}\left(Q^{2}\right)\right)$ expressions for the derivative of the heavy quark structure function, which are proportional to the singlet quark distribution, then as for the NLO derivative for $F_{2, H}\left(x, Q^{2}\right)$ we see that continuity is not achieved. The difference between the VFNS expression and the FFNS expression is

$$
2\left(\beta_{0}^{n_{f}}-\beta_{0}^{n_{f}+1}\right) C_{L, H q}^{\mathrm{FF}, \mathrm{PS}, 2}\left(Q^{2} / M^{2}\right) \otimes \Sigma_{0}^{n_{f}}\left(Q^{2}\right),
$$

where $C_{H q}^{\mathrm{FF}, \mathrm{PS}, 2}\left(z, Q^{2} / M^{2}\right)=C_{L, H q}^{\mathrm{VF}, \mathrm{PS}, 2}\left(z, Q^{2} / M^{2}\right)$. This NLO effect is very small, and as for $F_{2, H}\left(x, Q^{2}\right)$ the effect disappears as we work to higher orders.

For the light quark structure functions, there is one choice to make. There is a mass dependent contribution to the nonsinglet coefficient function at $\mathcal{O}\left(\alpha_{s}^{2}\left(Q^{2}\right)\right)$, but the form of the VFNS coefficient function is determined entirely by Eq. (3.8). In essence the mass dependent correction to $C^{\mathrm{NS}, n_{f}, 2}(z)$ contains a piece which becomes constant asymptotically, which represents $C^{\mathrm{NS}, n_{f}+1,2}(z)-C^{\mathrm{NS}, n_{f}, 2}(z)$, and a piece which grows like $\ln \left(Q^{2} / M^{2}\right)$ which takes account of the difference between the $n_{f}+1$ and $n_{f}$ flavor couplings. The $\mathcal{O}\left(\alpha_{s}^{3}\left(Q^{2}\right)\right) \ln Q^{2}$ derivative is slightly discontinuous at $Q^{2}$ $=M^{2}$, but this is corrected by inclusion of the $\mathcal{O}\left(\alpha_{s}^{3}\left(Q^{2}\right)\right)$ coefficient functions. For the pure singlet and gluon coefficient functions coupling to light partons, there are no mass dependent corrections in the FFNS at $\mathcal{O}\left(\alpha_{s, n_{f}}^{2}\left(Q^{2}\right)\right)$, and we simply use the same coefficient functions above and below $Q^{2}=M^{2}$. Continuity of the NLO structure functions is then automatic. However, the pure singlet coefficient function $C_{L, q H}^{\mathrm{VF}, \mathrm{PS}, 2}\left(z, Q^{2} / M^{2}\right)$ becomes nonzero at this order. It can be determined by demanding continuity of the $\ln Q^{2}$ derivative of the structure function in the gluon sector. This results in a similar procedure as for the heavy to heavy coefficient func- tion: the asymptotic form is put in by hand and multiplied by $\left(1-M^{2} / Q^{2}\right) v$, while there are nonzero terms at $Q^{2}=M^{2}$, which are multiplied by $v$ to ensure that they vanish as $Q^{2}=M^{2} \rightarrow \infty$. The calculation is straightforward, and we do not present details here. As for the heavy quark structure function, the $\mathcal{O}\left(\alpha_{s}^{3}\left(Q^{2}\right)\right) \ln Q^{2}$ derivative in the singlet quark sector is slightly discontinuous at $Q^{2}=M^{2}$, but again this is corrected at next order by inclusion of the $\mathcal{O}\left(\alpha_{s}^{3}\left(Q^{2}\right)\right)$ coefficient functions.

Now that the NLO prescription for the longitudinal structure function is completely defined we can examine the results. Using our NLO coefficient functions (7.18) and (7.11), and the NLO partons obtained from the best fit, we calculate the NLO charm quark longitudinal structure function. This is shown in Fig. 8 along with the continuation of the NLO FFNS expression and the NLO ZM-VFNS result. Once again the VFNS increases above the FFNS result very smoothly, despite the discontinuity in the $\ln Q^{2}$ derivative in the singlet quark sector, which is now demonstrably minute. At very high $Q^{2}$ our expression tends towards the ZM-VFNS as required, but as at LO the two become very different at lower $Q^{2}$. As in the case of $F_{2}\left(x ; Q^{2}\right)$ at NLO, the difference between the VFNS and the continuation of the FFNS is reduced compared to the difference at LO for the same reasons. Hence, we have every reason to consider our prescription for the longitudinal structure functions quite satisfactory.

In fact we can compare to some data. At $x<0.1$ the VFNS, ZM-VFNS, and FFNS values for the total $F_{L}\left(x, Q^{2}\right)$ are very similar. However, the NMC collaboration have produced data for $0.11>x>0.0045$ and $1.3 \mathrm{GeV}^{2}<Q^{2}$ $<20.6 \mathrm{GeV}^{2}$ [33], $Q^{2}$ increasing as $x$ increases. These data are in the region, where our VFNS prescription produces very different results to the ZM-VFNS (but almost identical to the FFNS) for $F_{L, c}\left(x, Q^{2}\right)$, and hence significantly different results for the total longitudinal structure function. Using the parton distributions obtained from our best global fit, we produce predictions for $R\left(x, Q^{2}\right)=F_{L}\left(x, Q^{2}\right) /\left(F_{2}\left(x, Q^{2}\right)\right.$ $\left.-F_{L}\left(x, Q^{2}\right)\right)$ using the ZM-VFNS and the VFNS, and compare data. The results are shown in Fig. 9. The kink in both curves at the lowest $x$ values comes about because for all data points other than that at $x=0.0045$, as $x$ decreases $Q^{2}$ also decreases smoothly, while for this point the extraction of $R\left(x, Q^{2}\right)$ relies on an extrapolation and the $Q^{2}$ is actually almost identical to that for the $x=0.008$ point. The kink in the ZM-VFNS expression at $Q^{2}=m_{c}^{2}=1.8 \mathrm{GeV}^{2}$ is due to all charm coefficient functions turning on discontinuously at this point. Comparing to the data it is clear that the ZM-VFNS curve is much too large for most of the $x$ range, while down to $x=0.01$ the VFNS curve matches the data quite well. Thus, there is strong evidence for including charm mass effects in the longitudinal structure function, and our prescription seems reasonably successful. Other VFNS prescriptions would lead to $R\left(x, Q^{2}\right)$ somewhere between the two curves. The theory is clearly below the data for the lowest $x$ and $Q^{2}$ points, where the charm contribution to $R\left(x, Q^{2}\right)$ is extremely small, i.e., the VFNS and ZM-VFNS curves are almost identical. The smallness of $R\left(x, Q^{2}\right)$, and the decrease with decreasing $x$ at constant $Q^{2}$ in this region are largely due to a negative small $x$ contribution from $C_{L, i g}^{n_{f}, 2}(z)$, which 

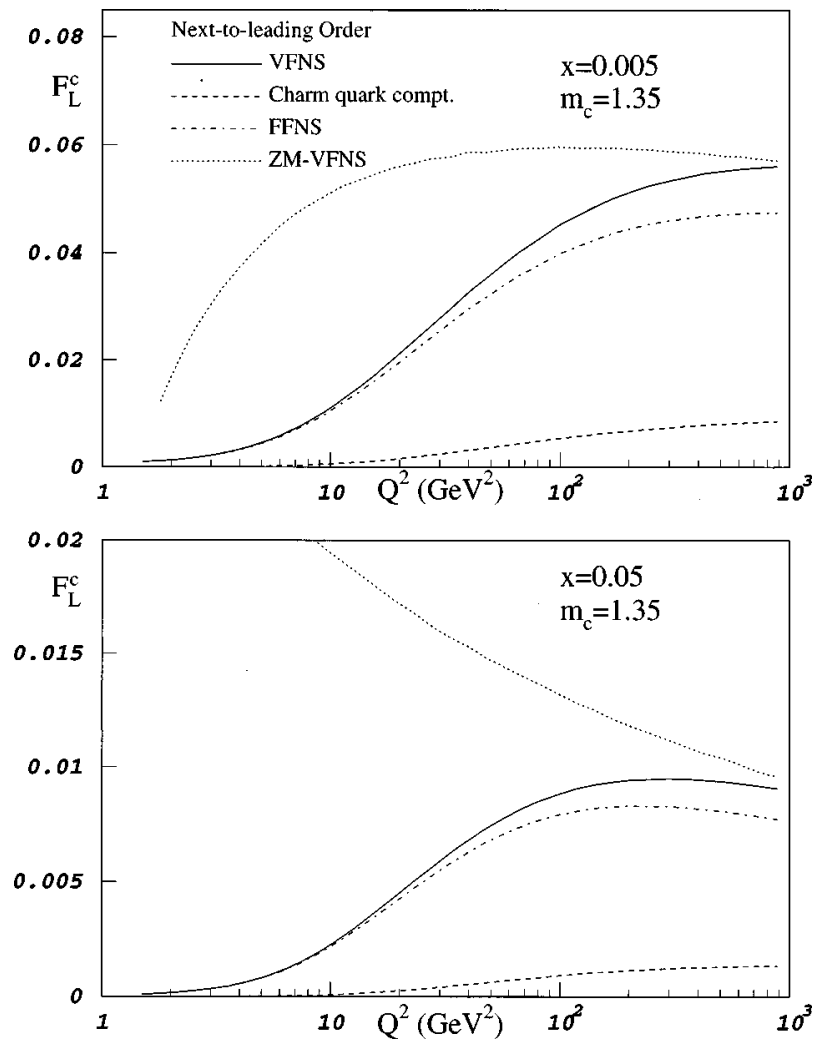

FIG. 8. Same as Fig. 7, but with NLO prescriptions and NLO parton distributions.

becomes increasingly important as $x$ and $Q^{2}$ fall. Thus, the difference between the theory and data for the two lowest $x$ points is perhaps a sign of the failure of the NLO-in- $\alpha_{s}\left(Q^{2}\right)$ calculation of structure functions at small $x .{ }^{12}$

As with $F_{2}\left(x, Q^{2}\right)$ the NLO calculation is the best that can be done explicitly with the present knowledge of structure functions. However, as in this previous case, we outline the procedure for all orders. The general form of the expressions is presented in Eqs. (7.1) and (7.2), and for the heavy quark structure function nothing essentially new compared to the LO and NLO prescriptions occurs. At $n$th nontrivial order we determine $C_{L, H H}^{\mathrm{VF}, n}\left(z, Q^{2} / M^{2}\right)$ by demanding continuity of the $\ln Q^{2}$ derivative of the structure function in the gluon sector, and by demanding the correct asymptotic form. At each order the correct asymptotic form will not appear in the continuity conditions and need to be introduced by hand. Each time we multiply by $\left(1-M^{2} / Q^{2}\right) v$. At each order there will also be terms introduced by the continuity demand which must vanish as $Q^{2} \rightarrow \infty$, and we multiply these by $M^{2} / Q^{2}$. At every order this determination of $C_{L, H H}^{\mathrm{VF}, n}\left(z, Q^{2} / M^{2}\right)$ predetermines $C_{L, H g}^{\mathrm{VF}, n+1}\left(z, Q^{2} / M^{2}\right)$ and

\footnotetext{
${ }^{12}$ The curve labeled $R_{\mathrm{QCD}}$ in Fig. 10 of the NMC paper [33] contains little information. For $F_{L}\left(x, Q^{2}\right)$ it uses a LO formula [34] (and hence does not contain the important NLO small $x$ effect), which assumes our massless quarks at all $Q^{2}$, along with a gluon which has been extracted using a NLO fit in the FFNS [2]. Moreover, this gluon is not constrained at large $x$ and is highly inconsistent with large $x$ data. From the momentum sum rule this means its form at small $x$ is also much different to a well constrained gluon.
}

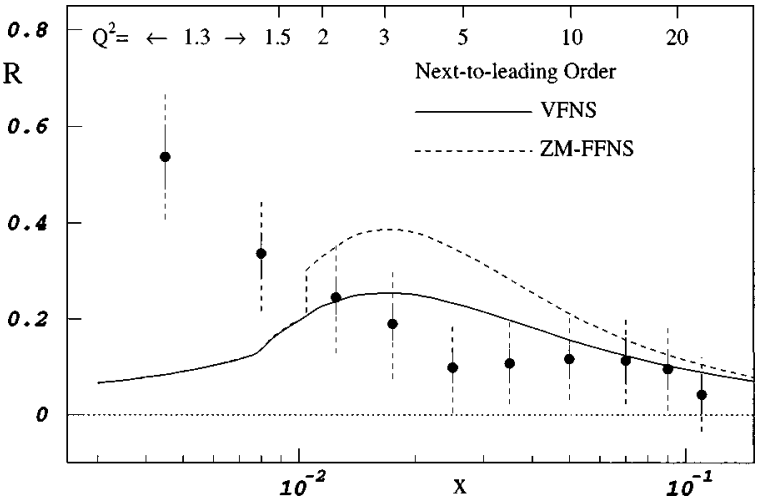

FIG. 9. Our prediction for $R\left(x, Q^{2}\right)$ using our NLO prescription, the NLO partons obtained from our global fit and $m_{c}=1.35 \mathrm{GeV}$ compared with the NMC data [33]. Also shown is the prediction obtained using the same parton distributions but for the NLO ZMVFNS prescription. The curves are computed using $Q^{2}=1.3 \mathrm{GeV}^{2}$ for $x \leqslant 0.0077$ and $Q^{2}=262 x^{1.09}$ for $x \geqslant 0.0077$.

$C_{L, H q}^{\mathrm{VF}, n+1}\left(z, Q^{2} / M^{2}\right)$ by using Eqs. (3.6) and (3.7) to $\mathcal{O}\left(\alpha_{s}^{n+1}\left(Q^{2}\right)\right)$. The comments concerning the separation of $C_{L, H H}^{\mathrm{VF}, n}\left(z, Q^{2} / M^{2}\right)$ into nonsinglet and pure singlet parts in Sec. IV apply again.

For the light quark structure function the procedure at higher orders is also straightforward. As with the $\mathcal{O}\left(\alpha_{s, n_{f}+1}^{2}\left(Q^{2}\right)\right)$ coefficient function we determine $C_{L, q H}^{\mathrm{VF}, n}\left(z, Q^{2} / M^{2}\right)$ by demanding continuity of the derivative of the light function in the gluon sector, analogously to the heavy quark sector. With this one degree of freedom eliminated in this way, all other VFNS coefficient functions are determined uniquely order by order in $\alpha_{s, n_{f}+1}\left(Q^{2}\right)$ by Eqs. (3.8)-(3.10), i.e., this determination of $C_{L, q H}^{\mathrm{VF}, n}\left(z, Q^{2} / M^{2}\right)$ predetermines $C_{L, q g}^{\mathrm{VF}, n+1}\left(z, Q^{2} / M^{2}\right)$ and $C_{L, q q}^{\mathrm{VF}, \mathrm{PS}, n+1}\left(z, Q^{2} / M^{2}\right)$ by using Eqs. (3.9) and (3.10) to $\mathcal{O}\left(\alpha_{s}^{n+1}\left(Q^{2}\right)\right)$.

Thus, we have completely defined our prescription for calculating the structure function $F_{L}\left(x, Q^{2}\right)$ order by order. As for $F_{2}\left(x, Q^{2}\right)$ we can sum it up in a simple diagram, shown in Table III. The generalization to the case of two heavy quarks follows the same lines as for the case of $F_{2}\left(x, Q^{2}\right)$ which was discussed at the end of Sec. V. For $Q^{2}<m_{b}^{2}$ the bottom quark effects are all treated via FFNS coefficient functions, while in the region above $Q^{2}=m_{b}^{2}$, we have a variable flavor scheme for both the charm and bottom quark. For high orders in $\alpha_{s}\left(Q^{2}\right)$ there will be mixing of the effects of the two quarks, but for the orders currently available in practice the mixing is extremely small indeed, as with $F_{2}\left(x, Q^{2}\right)$, and the bottom coefficient functions are essentially the same as those for charm with $m_{c} \rightarrow m_{b}$ and with five flavors rather than four.

Our prescription uniquely determines all VFNS coefficient functions, and as for $F_{2}\left(x, Q^{2}\right)$, while not leading to absolute correctly ordered expressions, it is a relatively simple prescription for obtaining order-by-order structure functions, which are very similar to the hypothetical strictly correct ones, which reduce to the correct asymptotic form order by order in $\alpha_{s, n_{f+1}}\left(Q^{2}\right)$, and which are consistent with physical requirements order by order. All ways of satisfying both Eqs. (3.6)-(3.10) and the correct asymptotic limits will 
TABLE III. Prescription for the order by order in $\alpha_{s}\left(Q^{2}\right)$ determination of the VFNS coefficient functions for $F_{L}\left(x, Q^{2}\right)$. In each case $C_{L, a H}^{\mathrm{VF}, n}\left(z, M^{2} / Q^{2}\right)$ is determined by introducing the asymptotic form multiplied by $\left(1-M^{2} / Q^{2}\right) v$ and multiplying the terms determined by continuity by $M^{2} / Q^{2}$.

\begin{tabular}{|c|c|c|}
\hline $\begin{array}{l}\text { Order of } \\
\text { equality }\end{array}$ & Eq. & Coefficient functions determined \\
\hline \multirow[t]{3}{*}{$\alpha_{s}\left(Q^{2}\right)$} & $(3.6)$ & $C_{L, H g}^{\mathrm{VF}, 1}$ \\
\hline & $(3.8)$ & $C_{L, q q}^{\mathrm{VF}, \mathrm{NS}, 1}$ \\
\hline & $(3.9)$ & $C_{L, q g}^{\mathrm{VF}, 1}$ \\
\hline \multirow[t]{5}{*}{$\alpha_{s}^{2}\left(Q^{2}\right)$} & $(3.6)$ & $\begin{array}{l}C_{L, H H}^{\mathrm{VF}, \mathrm{S}, 1} \text { [by continuity of }\left(d F_{L, H} / d \ln Q^{2}\right)_{M^{2}} \text { in gluon sector } \\
\left.\text { at } \mathcal{O}\left(\alpha_{s}^{2}\left(Q^{2}\right)\right)\right], C_{L, H g}^{\mathrm{VF}, 2}\end{array}$ \\
\hline & $(3.7)$ & $C_{L, H q}^{\mathrm{VF}, 2}$ \\
\hline & $(3.8)$ & $C_{L, q q}^{\mathrm{VF}, \mathrm{NS}, 2}$ \\
\hline & $(3.9)$ & $C_{L, q g}^{\mathrm{VF}, 2}$ \\
\hline & $(3.10)$ & $C_{L, q q}^{\mathrm{VF}, \mathrm{PS}, 2}$ \\
\hline \multirow[t]{5}{*}{$\alpha_{s}^{3}\left(Q^{2}\right)$} & $(3.6)$ & $\begin{array}{l}C_{L, H H}^{\mathrm{VF}, 2}\left[\text { by continuity of }\left(d F_{L, H} / d \ln Q^{2}\right)_{M^{2}} \text { in gluon sector }\right. \\
\left.\text { at } \mathcal{O}\left(\alpha_{s}^{3}\left(Q^{2}\right)\right)\right], C_{L, H g}^{\mathrm{VF}, 3}\end{array}$ \\
\hline & $(3.7)$ & $C_{L, H q}^{\mathrm{VF}, 3}$ \\
\hline & $(3.8)$ & $C_{L, q q}^{\mathrm{VF}, 3}$ \\
\hline & $(3.9)$ & $\begin{array}{l}C_{L, q H}^{\mathrm{VF}, 2} \text { [by continuity of }\left(d F_{L, i} / d \ln Q^{2}\right)_{M^{2}} \text { in gluon sector } \\
\left.\text { at } \mathcal{O}\left(\alpha_{s}^{3}\left(Q^{2}\right)\right)\right], C_{L, q g}^{\mathrm{VF}, 3}\end{array}$ \\
\hline & $(3.10)$ & $C_{L, q q}^{\mathrm{VF}, \mathrm{PS}, 3}$ \\
\hline$\cdots$ & $\cdots$ & $\cdots$ \\
\hline \multirow[t]{5}{*}{$\alpha_{s}^{n}\left(Q^{2}\right)$} & $(3.6)$ & $\begin{array}{l}C_{L, H H}^{\mathrm{VF}, \mathrm{S}, n-1}\left[\text { by continuity of }\left(d F_{L, H} / d \ln Q^{2}\right)_{M^{2}} \text { in gluon }\right. \\
\left.\text { sector at } \mathcal{O}\left(\alpha_{s}^{n}\left(Q^{2}\right)\right)\right], C_{L, H g}^{\mathrm{VF}, n}\end{array}$ \\
\hline & $(3.7)$ & $C_{L, H q}^{\mathrm{VF}, n}$ \\
\hline & $(3.8)$ & $C_{L, q q}^{\mathrm{VF}, n}$ \\
\hline & $(3.9)$ & $\begin{array}{l}C_{L, q H}^{\mathrm{VF}, n-1}\left[\text { by continuity of }\left(d F_{L, i} / d \ln Q^{2}\right)_{M^{2}} \text { in gluon }\right. \\
\left.\text { sector at } \mathcal{O}\left(\alpha_{s}^{n}\left(Q^{2}\right)\right)\right], C_{L, q g}^{\mathrm{VF}, n}\end{array}$ \\
\hline & $(3.10)$ & $C_{L, q q}^{\mathrm{VF}, \mathrm{PS}, n}$ \\
\hline
\end{tabular}

be correct in a certain sense (provided they are consistent with ordering within a given renormalization scheme), but many will have behavior which is unsatisfactory for $Q^{2}$ not much larger than $M^{2}$, and we have seen an example of this. As with $F_{2}\left(x, Q^{2}\right)$ we believe our prescription to be very suitable.

\section{SUMMARY AND CONCLUSION}

In this paper we have constructed an order by order in $\alpha_{s}$ prescription for calculating the neutral current structure function including the effects of a massive quark. For the region $Q^{2}<M^{2}$ this has essentially just been the normal FFNS, where the heavy quark is not treated as a constituent of the hadron, but all heavy quarks in the final state are generated via the electroweak boson interacting with light partons. For $Q^{2}>M^{2}$ we have to solve the problem of summing large logs in $Q^{2} / M^{2}$ and $\mu^{2} / M^{2}$, which appear at all orders in $\alpha_{s}\left(\mu^{2}\right)$. The easiest way to do this is to to treat the heavy quark as a parton, in which case the logs will be summed automatically, when one solves the evolution equations for the partons. If one chooses the parton distributions above $\mu^{2}=M^{2}$ to evolve as though massless and in the $\overline{\mathrm{MS}}$ scheme, then the new $n_{f}+1$ flavor parton distributions are determined in terms of the FFNS parton distributions at all $\mu^{2}$ by well-defined, calculable matrix elements, which contain logs in $\mu^{2} / M^{2}$. In particular the heavy quark distribution is determined entirely in terms of the light parton distributions. The matrix elements can then be used to define the $n_{f}+1$ flavor parton distributions in terms of the $n_{f}$ flavor distributions at some scale (in practice $\mu^{2}=M^{2}$ is by far the most convenient), and the evolution upwards can take place in terms of $n_{f}+1$ massless flavors with the correct asymptotic limits being guaranteed. If the massless $n_{f}+1$ flavor coefficients functions are used, then the correct asymptotic limit for the structure functions is also reached.

The main problem lies in obtaining the correct description in the region not too far above $Q^{2}=M^{2}$. We have demonstrated that this is achieved to all orders by defining the mass-dependent coefficient functions above $Q^{2}=M^{2}$ in terms of the operator matrix elements and the FFNS coefficient functions as in Eqs. (3.6)-(3.10). However, we have also demonstrated that since there are more degrees of freedom on the right-hand side of these equations than on the left, the additional ones all being coefficient functions coupling to heavy quarks, there is freedom in precisely how the coefficient functions may be chosen. Although in a true wellordered calculation, this ambiguity disappears, this manner of ordering is at the very least extremely complicated, in- 
volving parts of the FFNS from all orders in $\alpha_{s}\left(\mu^{2}\right)$ at each order in the calculation, and in practice is probably impossible, there being no unique prescription for ordering the $\mathcal{O}\left(M^{2} / Q^{2}\right)$ terms. Hence we choose to order our calculation as in the normal order by order in $\alpha_{s}\left(\mu^{2}\right)$ manner, choosing the very simple natural scale $\mu^{2}=Q^{2}$, which puts all of the mass effects into the coefficient functions and guarantees the correct asymptotic limit order by order in $\alpha_{s}\left(Q^{2}\right)$. We then determine the precise form of our heavy quark coefficient functions by demanding continuity, not only of the structure functions at $Q^{2}=M^{2}$ (which is automatic), but also the continuity of the $\ln Q^{2}$ derivative of the structure function. In practice this exact continuity is only possible for those terms proportional to the gluon, but this is by far the dominant contribution. Our constraint then determines our prescription for dealing with heavy quarks completely, and incorporates the correct qualitative threshold behavior into every coefficient function at each order of $\alpha_{s}\left(Q^{2}\right)$, not relying on cancellations between terms with incorrect behavior and of different orders to obtain satisfactory results. In practice the most important of our results are the zeroth-order coefficient function for $F_{2, c}\left(x, Q^{2}\right)$, Eq. (4.6), which exhibits the correct threshold behavior in $\hat{W}^{2}$ as well as reducing to the correct asymptotic form, and the absence of a zeroth-order coefficient function for $F_{L, c}\left(x, Q^{2}\right)$, the $\mathcal{O}\left(\alpha_{s}\left(Q^{2}\right)\right)$ coefficient functions being Eqs. (7.5) and (7.10), which again exhibit the correct threshold behavior and asymptotic limits.

We display the results obtained using our prescription for neutral current structure functions in Figs. 3, 4, 7, and 8, finding that they exhibit exactly the type of behavior we would expect, i.e., smoothly deviating from the FFNS at low $Q^{2}$, and tending towards the $n_{f}+1$ massless results at high $Q^{2}$, in all cases. In particular we notice that the bump in the charm quark longitudinal structure function at $Q^{2}$ $\approx 10 \mathrm{GeV}^{2}$, which occurs in other variable flavor number schemes is absent here. We also see that our predictions agree very well with the current data on the charm structure function which exists from $1.5 \mathrm{GeV}^{2}<Q^{2}<100 \mathrm{GeV}^{2}$, implying a charm quark mass of $\sim 1.45 \mathrm{GeV}$. We note that comparisons of theoretical predictions with the complete range of data on the charm structure function appear very rarely (in particular, detailed comparison with EMC data is frequently omitted), and we strongly encourage this as the best constraint on any theory.

The general technique can be applied to all other quantities in perturbative QCD, which require the convolution of coefficient functions with parton distributions. We can always choose the parton distributions to evolve as though there are $n_{f}+1$ massless flavors in the $\overline{\mathrm{MS}}$ scheme, factor these into the mass dependent operator matrix elements and the FFNS parton distributions, and then obtain the coefficient functions in the variable flavor scheme in terms of those in the fixed flavor scheme by equating the parts proportional to each FFNS parton distribution. Indeed, the expressions (3.6)-(3.10) are not exclusive to neutral current structure functions, but apply to all quantities, which can be written as the sum of convolutions of coefficient functions with single parton distributions. In the Appendix we discuss the case of the charged current structure functions as an example. For expressions involving more than one parton distribution, the generalization is clear, e.g., for proton-proton scattering the
FFNS and VFNS coefficients are related by equations of the form $C_{i a b}^{\mathrm{FF}}=C_{i c d}^{\mathrm{VF}} A^{c a} A^{d b}$. In all cases there will be ambiguity in definitions of the heavy parton coefficient functions, but these can always be eliminated by demanding as much continuity of the $\ln Q^{2}$-derivative order by order in $\alpha_{s}\left(Q^{2}\right)$ as possible.

Let us briefly discuss problems which arise in other approaches to heavy quark structure functions. Buza et al. do not provide a detailed prescription for the region of $Q^{2}$ just above $M^{2}$. They have a means of extrapolating the structure function from the FFNS result at $Q^{2}<M^{2}$ to the ZM-VFNS result at $Q^{2} / M^{2} \rightarrow \infty$ in a way which guarantees smoothness $[14,15]$, but it seems phenomenologically motivated, with no strict definition of the ordering and certainly no expressions for parton distributions and coefficient functions in the intermediate region. The ACOT group have a prescription, which involves switching from $n_{f}$ to $n_{f}+1$ massless flavors in the evolution, and a way of determining the VFNS coefficient functions $[22,23]$ which at low orders appears to be the same as prescribed in Eqs. (3.6)-(3.10). However, their way of eliminating the free choices in the heavy quark coefficient functions involves assuming that the behavior is as if there is intrinsic charm in the proton at all scales above the transition point, rather than charm being generated almost entirely from the gluon. This leads to coefficient functions having thresholds in $Q^{2}=M^{2}$ rather than $\hat{W}^{2}=4 M^{2}$, and a mixing of orders being required (and a complicated renormalization scale being advantageous) in order to ensure cancellations and that smooth behavior occurs, e.g., the $\mathcal{O}\left(\alpha_{s}\left(\mu^{2}\right)\right)$ gluon coefficient function must appear at the same time as the zeroth-order quark coefficient function. This mixing of orders is incorrect, being at odds with well-ordered asymptotic expressions, but removing it results in a lack of smoothness in the structure functions. Even when this mixing is retained, the behavior of the longitudinal structure function is still not smooth. The MRRS procedure [28] is based on the leading log limit of Feynman diagrams, rather than the renormalization group and as such incorporates mass-dependent effects in the evolution, but seems more difficult to define formally to all orders in $\alpha_{s}$. The definition of the heavy quark coefficient functions uses similar reasoning to ACOT, but in this case with ordering such that it reduces to the correct wellordered form asymptotically. These coefficient functions along with the imposition of this correct ordering lead to an unphysical lack of smoothness in the structure functions (which is made slightly worse by the mass-dependent contributions to the evolution), particularly for the longitudinal structure function. Our prescription has none of the above problems. It is well defined to all orders, reduces to correct well-ordered expressions at both low and high $Q^{2}$, and exhibits precisely the behavior one would expect. Hence, we believe that our prescription is the best currently available to describe the heavy quark contribution to structure functions.

Before finishing let us mention a couple of points in which our treatment is incomplete. Firstly, we have assumed that there is no intrinsic charm in the nucleon. Equation (2.1) is formally correct up to the quoted error, but this error has an unknown numerical factor and may be enhanced by functions of $x$. It appears that for intrinsic charm the numerical factor of this "higher twist" correction is rather large and that the contribution is enhanced by a factor of $(1-x)^{-1}$. 
Therefore, at large $x$, where the leading twist contribution to the charm structure function is not large anyway, it seems as though the "higher twist" intrinsic charm may constitute an important part of the total charm structure function [35]. The treatment of this correction is outside the scope of this paper, which deals only with the "leading twist" contribution to the structure function, and we believe it is not naturally dealt with in any other VFNS. However, it seems very unlikely that in most of the region, where there is current data on the charm structure function, or where the charm contribution is a sizable fraction of the total structure function, that this "higher twist" contribution plays any significant role at all. Using the type of values expected for this intrinsic charm (see, e.g., [36]), then adding to our values does bring the $x$ $=0.422$ prediction in line with the EMC data point, raises the $x=0.237$ predictions quite significantly (but neither really helps or hinders the comparison to the three data points), raises the $x=0.133$ predictions a little (tending to make the comparison a little worse), and has negligible effect for lower $x$. Hence, the $x=0.422, Q^{2}=78 \mathrm{GeV}^{2}$ EMC data point may be seen as some evidence for this "higher twist" intrinsic charm.

Finally we note that throughout this paper, we have completely ignored the problem of enhancement of higher orders in $\alpha_{s}$ by $\ln (1 / x)$ terms. These terms certainly do have the potential to alter quantitatively the results of this paper. Correctly including the leading $\ln (1 / x)$ terms within the context of only massless quarks is a complicated procedure, though it does appear to improve the description of small $x$ data [20]. Some results on heavy quark coefficient functions, which include leading $\ln (1 / x)$ terms already exist $[37,38]$. It would clearly be desirable to extend this work and to include both the correct treatment of leading $\ln (1 / x)$ terms and a correct description of heavy quark results within a single framework. Work along these lines is currently in progress.

\section{APPENDIX: CHARGED CURRENT STRUCTURE FUNCTIONS}

The treatment of the charged current structure function follows exactly the same reasoning as for the neutral current case. Let us consider $F_{2}\left(x, Q^{2}\right)$. Equations (3.6)-(3.10) are derived in exactly the same way, but now take a different form because there are no nonsinglet coefficient functions. For the case where a heavy quark is produced directly by the interaction with the $W$ boson, which we call the heavy quark structure function, we have

$$
C_{H g}^{\mathrm{FF}, \mathrm{S}}=C_{H g}^{\mathrm{VF}, \mathrm{S}} \otimes A_{g g, H}^{\mathrm{S}}+n_{f} C_{H q}^{\mathrm{VF}, \mathrm{PS}} \otimes A_{q g, H}^{\mathrm{S}}+C_{H H}^{\mathrm{VF}, \mathrm{PS}} \otimes A_{H g}^{\mathrm{S}},
$$

and

$$
\begin{aligned}
C_{H q}^{\mathrm{FF}, \mathrm{S}}= & C_{H H}^{\mathrm{VF}, \mathrm{PS}} \otimes A_{H q}^{\mathrm{PS}}+C_{H q}^{\mathrm{VF}, \mathrm{PS}} \otimes\left[A_{q q, H}^{\mathrm{NS}}+n_{f} A_{q q, H}^{\mathrm{PS}}\right]+C_{H g}^{\mathrm{VF}, \mathrm{S}} \\
& \otimes A_{g q, H}^{\mathrm{S}} .
\end{aligned}
$$

We note that what we have denoted the charm quark structure function here may be interpreted physically as the unlike sign dimuon contribution. In the case where the $W$ boson directly produces a light quark, which we call the light quark structure function, we have

$$
\begin{aligned}
C_{q g}^{\mathrm{S}}+C_{q g}^{\mathrm{FF}, \mathrm{S}}= & C_{q g}^{\mathrm{VF}, \mathrm{S}} \otimes A_{g g, H}^{\mathrm{S}}+n_{f} C_{q q}^{\mathrm{VF}, \mathrm{PS}} \otimes A_{q g, H}^{\mathrm{S}}+C_{q H}^{\mathrm{VF}, \mathrm{PS}} \\
& \otimes A_{H g}^{\mathrm{S}},
\end{aligned}
$$

and

$$
\begin{aligned}
C_{q q}^{\mathrm{PS}}+C_{q q}^{\mathrm{FF}, \mathrm{PS}}= & n_{f} C_{q q}^{\mathrm{VF}, \mathrm{PS}} \otimes A_{q q, H}^{\mathrm{PS}}+C_{q H}^{\mathrm{VF}, \mathrm{PS}} \otimes A_{H q}^{\mathrm{PS}}+C_{q g}^{\mathrm{VF}, \mathrm{S}} \\
& \otimes A_{g q, H}^{\mathrm{S}} .
\end{aligned}
$$

It is not only the absence of the nonsinglet coefficient functions which is different, the ordering of the other coefficient functions also changes, in particular $C_{2, q_{i} q_{j}}^{\mathrm{PS}}, i \neq j$, begins at zeroth order. This changes the form of the relationship between the FFNS and the VFNS coefficient functions. For example, examination of (A1) reveals that we have the trivial equality

$$
C_{2, H g}^{\mathrm{FF}, 1}\left(z, Q^{2} / M^{2}\right) \equiv C_{2, H g}^{\mathrm{FF}, 1}\left(z, Q^{2} / M^{2}\right),
$$

whereas now we have the nontrivial relationship

$$
\begin{aligned}
C_{2, q g}^{\mathrm{FF}, 1}\left(z, Q^{2} / M^{2}\right)= & C_{2, q g}^{\mathrm{VF}, 1}\left(z, Q^{2} / M^{2}\right)-\left(\ln \left(Q^{2} / M^{2}\right)+c_{\mathrm{rs}}\right) P_{q g}^{0} \\
& \otimes C_{2, q H}^{\mathrm{VF}, \mathrm{PS}, 0},
\end{aligned}
$$

e.g., the zeroth-order coefficient function for a charm quark to interact with a $W^{-}$to produce a strange quark is undetermined. As in the previous case we determine this zerothorder heavy quark coefficient function by demanding continuity of the $\ln Q^{2}$ derivative of the structure function, in the gluon sector (again at lowest order we have complete continuity), along with demanding the correct asymptotic result. Unlike the neutral current case, this time it is the strange quark (or down quark) structure function on which the condition is imposed, rather than the charm quark structure function. This is because at lowest order the charm quark structure function is completely independent of the charm quark distribution, whereas the light quark structure functions do depend on it. However, in complete analogy with the neutral current case, our constraint results in

$$
C_{2, q H}^{\mathrm{VF}, \mathrm{PS}, 0}\left(Q^{2} / M^{2}\right) \otimes P_{q g}^{0}=\frac{d C_{2, q g}^{\mathrm{FF}, 1}\left(z, Q^{2} / M^{2}\right)}{\ln \left(Q^{2}\right)},
$$

where the left-hand side automatically has the correct threshold behavior and the right-hand side $\rightarrow P_{q g}^{0}(z)$ as $Q^{2} / M^{2} \rightarrow \infty$. Using this explicitly in (A6) then results in the $C_{2, q g}^{\mathrm{VF}, 1}\left(z, Q^{2} / M^{2}\right)$ reducing to the correct massless $\overline{\mathrm{MS}}$ limit as $Q^{2} / M^{2} \rightarrow \infty$, as it must by construction.

This procedure can be repeated at all orders in exactly the same way as for the neutral current structure function. This time there are only two coefficient functions to be determined, $C_{2, H q}^{\mathrm{VF}, \mathrm{PS}}\left(z, Q^{2} / M^{2}\right)$ as we have just seen, and which exists at all orders, and $C_{2, H H}^{\mathrm{VF}, \mathrm{PS}}\left(z, Q^{2} / M^{2}\right)$. The latter begins at $\mathcal{O}\left(\alpha_{s}^{2}\left(Q^{2}\right)\right)$ and will be determined by demanding continuity of the $\ln Q^{2}$ derivative of the structure function, where a heavy quark is produced directly at the interaction vertex of the $W$ boson at $\mathcal{O}\left(\alpha_{s}^{3}\left(Q^{2}\right)\right)$. The extension to the longitudinal charged current structure function is also easily achieved using the above results and the discussion of the longitudinal neutral current structure function in Sec. VII. 
[1] J. C. Collins, D. E. Soper, and G. Sterman, in Perturbative Quantum Chromodynamics, edited by A. H. Mueller (World Scientific, Singapore, 1989), and references therein.

[2] H1 Collaboration, S. Aid et al., Nucl. Phys. B470, 3 (1996); B497, 3 (1997).

[3] ZEUS Collaboration, M. Derrick et al., Z. Phys. C 69, 607 (1996); 72, 399 (1996).

[4] H1 Collaboration, C. Adloff et al., Z. Phys. C 72, 593 (1996).

[5] ZEUS Collaboration, J. Breitweg et al., Phys. Lett. B 407, 402 (1997).

[6] E. Witten, Nucl. Phys. B104, 445 (1976).

[7] M. Glück, E. Reya, and M. Stratmann, Nucl. Phys. B422, 37 (1994); M. Glück, E. Reya, and A. Vogt, Z. Phys. C 67, 433 (1995).

[8] M. A. Shifman, A. I. Vainshtein, and V. I. Zakharov, Nucl. Phys. B136, 157 (1978).

[9] M. Glück and E. Reya, Phys. Lett. 83B, 98 (1979).

[10] E. Laenen, S. Riemersma, J. Smith, and W. L. van Neerven, Nucl. Phys. B392, 162 (1993); B. W. Harris and J. Smith, ibid. B452, 109 (1995).

[11] J. C. Collins and W. K. Tung, Nucl. Phys. B278, 934 (1986).

[12] H. L. Lai et al., Phys. Rev. D 55, 1280 (1997).

[13] A. D. Martin, R. G. Roberts, and W. J. Stirling, Phys. Rev. D 50, 6734 (1994).

[14] M. Buza et al., Nucl. Phys. B472, 611 (1996).

[15] M. Buza et al., Eur. Phys. J. C1, 301 (1998).

[16] M. Buza et al., Phys. Lett. B 411, 211 (1997).

[17] J. C. Collins, F. Wilczek, and A. Zee, Phys. Rev. D 18, 242 (1978).

[18] W. Bernreuther and W. Wetzel, Nucl. Phys. B197, 228 (1982); W. Bernreuther, Ann. Phys. (N.Y.) 151, 127 (1983).

[19] S. A. Larin, T. van Ritbergen, and J. A. M. Vermaseren, Nucl. Phys. B438, 278 (1995).

[20] R. Thorne, Nucl. Phys. B512, 323 (1998).

[21] F. I. Olness and W. K. Tung, Nucl. Phys. B308, 813 (1988).

[22] M. Aivazis, F. Olness, and W. K. Tung, Phys. Rev. D 50, 3085 (1994).

[23] M. Aivazis, J. C. Collins, F. Olness, and W. K. Tung, Phys. Rev. D 50, 3102 (1994).
[24] J. C. Collins (in preparation).

[25] P. Agrawal, F. I. Olness, S. T. Riemersma, and W. K. Tung, Proceedings of the XXXth Rencontres de Moriond, "QCD and High Energy Hadronic interactions,', Les Arcs, France, 1995 (Editions Frontieres, Gif-sur-yvette, 1995), p. 353; C. Schmidt, in Deep Inelastic Scattering and QCD: 5th International Workshop, Proceedings of the International Workshop on Deep Inelastic Scattering and QCD (DIS97), Chicago, IL, 1997, AIP Conf. Proc. No. 407 (AIP, New York, 1997).

[26] J. Smith, in Proceedings of New Trends in HERA Physics, Ringberg, 1997 (in press), hep-ph/9708212.

[27] H. L. Lai and W. K. Tung, Z. Phys. C 74, 463 (1997).

[28] A. D. Martin, R. G. Roberts, M. G. Ryskin, and W. J. Stirling, Report No. RAL-TR-96-103, hep-ph/9612449 [Z. Phys. C (to be published)].

[29] S. Riemersma, J. Smith, and W. L. van Neerven, Phys. Lett. B 347, 143 (1995).

[30] A. D. Martin, R. G. Roberts, and W. J. Stirling, Phys. Lett. B 387, 419 (1996).

[31] R. S. Thorne, Phys. Lett. B 392, 463 (1997).

[32] EMC Collaboration, J. J. Aubert et al., Nucl. Phys. B213, 31 (1983).

[33] NMC Collaboration, M. Arneodo et al., Nucl. Phys. B483, 3 (1997).

[34] G. Altarelli and G. Martinelli, Phys. Lett. 76B, 89 (1978).

[35] S. J. Brodsky, P. Hoyer, A. H. Mueller, and W. K. Tang, Nucl. Phys. B369, 519 (1992), and references therein.

[36] B. W. Harris, J. Smith, and R. Vogt, Nucl. Phys. B461, 181 (1996).

[37] S. Catani, M. Ciafaloni, and F. Hautmann, Phys. Lett. B 242, 97 (1990); Nucl. Phys. B366, 135 (1991).

[38] S. Catani, Z. Phys. C 75, 665 (1997); Proceedings of the International Workshop on Deep Inelastic Scattering, Rome, 1996 (World Scientific, Singapore, 1997), p. 165.

[39] BCDMS Collaboration, A. C. Benvenuti et al., Phys. Lett. B 223, 485 (1989).

[40] L. W. Whitlow et al., Phys. Lett. B 282, 475 (1992).

[41] E665 Collaboration, M. R. Adams et al., Phys. Rev. D 54, 3006 (1996). 